\title{
MALT90 molecular content on high-mass IR-dark clumps ${ }^{\star}$
}

\author{
Gozde Saral $^{1}$, Marc Audard ${ }^{1}$, and Yuan Wang ${ }^{2}$ \\ 1 Department of Astronomy, University of Geneva, Chemin d'Ecogia 16, 1290 Versoix, Switzerland \\ e-mail: gozde. saral@unige.ch \\ 2 Max Planck Institute for Astronomy, Königstuhl 17, 69117 Heidelberg, Germany
}

Received 7 March 2018 / Accepted 15 October 2018

\begin{abstract}
Context. High mass stars form in groups or clusters in dense molecular clumps with sizes of $1 \mathrm{pc}$ and masses of $200 M_{\odot}$. Infrared-dark clumps and the individual cores within them with sizes $<0.1 \mathrm{pc}$ and masses $<100 M_{\odot}$ are important laboratories for high-mass star formation in order to study the initial conditions.

Aims. We investigate the physical and chemical properties of high-mass clumps in order to better understand the early evolutionary stages and find targets that show star formation signs such as infall motions or outflows.

Methods. We selected the high-mass clumps from ATLASGAL survey that were identified as dark at $8 / 24 \mu$ m wavelengths. We used MALT90 Survey data which provides a molecular line set $\left(\mathrm{HCO}^{+}, \mathrm{HNC}, \mathrm{HCN}, \mathrm{N}_{2} \mathrm{H}^{+}, \mathrm{H}^{13} \mathrm{CO}^{+}, \mathrm{HN}^{13} \mathrm{C}, \mathrm{SiO}\right)$ to investigate the physical and chemical conditions in early stages of star formation.

Results. Eleven sources have significant $\mathrm{SiO}$ detection (over $3 \sigma$ ) which usually indicates outflow activity. Thirteen sources are found with blue profiles in both or either $\mathrm{HCO}^{+}$and/or $\mathrm{HNC}$ lines and clump mass infall rates are estimated to be in the range of $0.2 \times 10^{-3} M_{\odot} \mathrm{yr}^{-1}-1.8 \times 10^{-2} M_{\odot} \mathrm{yr}^{-1}$. The excitation temperature is obtained as $<24 \mathrm{~K}$ for all sources. The column densities for optically thin lines of $\mathrm{H}^{13} \mathrm{CO}^{+}$and $\mathrm{HN}^{13} \mathrm{C}$ are in the range of $0.4-8.8\left(\times 10^{12}\right) \mathrm{cm}^{-2}$, and $0.9-11.9\left(\times 10^{12}\right) \mathrm{cm}^{-2}$, respectively, while it is in the range of $0.1-7.5\left(\times 10^{14}\right) \mathrm{cm}^{-2}$ for $\mathrm{HCO}^{+}$and $\mathrm{HNC}$ lines. The column densities for $\mathrm{N}_{2} \mathrm{H}^{+}$were ranging between $4.4-$ $275.7\left(\times 10^{12}\right) \mathrm{cm}^{-2}$ as expected from cold dense regions. Large line widths of $\mathrm{N}_{2} \mathrm{H}^{+}$might indicate turbulence and large line widths of $\mathrm{HCO}^{+}, \mathrm{HNC}$, and $\mathrm{SiO}$ indicate outflow activities. Mean optical depths are 20.32, and 23.19 for optically thick $\mathrm{HCO}^{+}$and $\mathrm{HCN}$ lines, and 0.39 and 0.45 for their optically thin isotopologues $\mathrm{H}^{13} \mathrm{CO}^{+}$and $\mathrm{HN}^{13} \mathrm{C}$, respectively.

Conclusions. This study reveals the physical and chemical properties of 30 high-mass IR-dark clumps and the interesting targets among them based on their emission line morphology and kinematics.
\end{abstract}

Key words. stars: formation - stars: massive - ISM: lines and bands - ISM: abundances - ISM: molecules - submillimeter: ISM

\section{Introduction}

High-mass stars $\left(L>10^{3} L_{\odot}\right)$ play a vital role in the star formation process from their birth to death, yet their formation is less understood than that of low-mass stars (e.g., Beuther et al. 2007; Zinnecker \& Yorke 2007). Since they form and evolve quickly and ionize and disrupt their natal molecular cloud by strong winds and intense UV radiation, observations of their earliest evolutionary phases are very difficult. In addition, the large distances and dense material in the molecular clouds require high angular resolution and high sensitivity observations which make systematic surveys difficult. Therefore, there is no well established evolutionary sequence for high-mass stars. Based on observational and theoretical studies the following sequence has been proposed: massive starless cores, gravitationally bound prestellar cores or infrared-dark clouds as the first phase; highmass protostellar objects or hot molecular cores as the second phase; and HII regions as the final phase (see the recent review: Motte et al. 2018). In order to better understand the initial conditions of star formation, recent studies focused on mainly nearby prestellar cores and low-mass infrared dark clouds (IRDCs) to study the morphology of the cores, as well as their temperature structures and chemical properties. While these observations had sensitivity and resolution limitations, new instruments like

\footnotetext{
* The reduced datacubes are only available at the CDS via anonymous ftp to cdsarc.u-strasbg.fr $(130.79 .128 .5)$ or via http: //cdsarc.u-strasbg.fr/viz-bin/qcat?J/A+A/620/A158
}

the IRAM30 and APEX telescopes and interferometers like the SMA, CARMA, and ALMA have access to high sensitivity and resolution observations.

Until recently it has been thought that most high-mass stars form in clustered environments within dense molecular clouds along with lower-mass stars (Motte et al. 1998; Lada \& Lada 2003; di Francesco et al. 2007) and recently interferometric observations of IRDCs revealed that these clumps have sizes of $\sim 1 \mathrm{pc}$ and masses of $\sim 200 M_{\odot}$ and they host very young starforming cores (e.g., Beuther et al. 2005). On the other hand, it is the dense cores (with sizes $<0.1 \mathrm{pc}$ and masses $<100 M_{\odot}$ ) within these clumps which are forming protostars or binaries. Also, high angular resolution observations reveal that the individual prestellar cores in clusters are more compact than isolated cores with scales of $0.02-0.03 \mathrm{pc}$ and they do not show further subfragmentation (Beuther et al. 2015). Although several surveys were performed to study the early evolutionary stages of high-mass stars, they targeted mainly the later stages such as HII regions (e.g., CORNISH, Hoare et al. 2012) and massive young stellar objects (MYSOs; e.g., rms, Urquhart et al. 2008). Within this context, to study the earlier stages and have a more complete picture of high-mass star formation, the APEX Telescope Large Area Survey ${ }^{1}$ (ATLASGAL, Schuller et al. 2009)

\footnotetext{
1 APEX is a collaboration between the Max-Planck-Institut für Radioastronomie, the European Southern Observatory, and the Onsala Space Observatory.
} 
mapped the dust continuum emission at $870 \mu \mathrm{m}$ systematically, in the inner Galactic plane over several $100 \mathrm{deg}^{2}$ with a uniform sensitivity. The ATLASGAL Survey identified and classified over 5000 compact sources (Contreras et al. 2013; Csengeri et al. 2014) at early evolutionary stages from quiescent clumps and protostellar cores to HII regions and PDRs in a series of papers (Schuller et al. 2009; Giannetti et al. 2014; König et al. 2017; Csengeri et al. 2016). Later, a subsample of ATLASGAL sources were observed with the $22 \mathrm{~m}$ single-dish Australia Telescope National Facility (ATNF) MOPRA Telescope $^{2}$, MALT90 Survey to examine their chemical and physical properties as well as evolutionary phases (Jackson et al. 2013; Hoq et al. 2013; Foster et al. 2013, 2011; Miettinen 2014; Rathborne et al. 2016).

In this study, we began an investigation of high-mass dense clumps in the ATLASGAL survey which were also observed in the MALT90 Survey, have high masses $\left(>100 M_{\odot}\right)$, but do not have any infrared counterparts in the IRAC $8 \mu \mathrm{m}$ or MIPS $24 \mu \mathrm{m}$ band, indicating that they are at very early stages of star formation (Giannetti et al. 2014). Based on these criteria, 30 infrared dark clouds were selected and their physical and chemical properties were studied in order to better understand the initial conditions during the early evolutionary phases of highmass stars. Spectral line profiles are examined in order to determine the infall signatures within the clumps which is one of the crucial signs of early stages of star formation (Wu et al. 2007; Beltrán et al. 2006; Rygl et al. 2013) and especially for massive star formation (McKee \& Tan 2003). The line profiles of $\mathrm{HCO}^{+}$, and $\mathrm{H}^{13} \mathrm{CO}^{+}$are generally used to study infall and outflow activities in star-forming regions (Wu et al. 2007; Chen et al. 2010; Csengeri et al. 2011; Rygl et al. 2013; Smith et al. 2013). Double-peaked lines with stronger blue or stronger red profiles, or single-peaked lines with blue- or red-skewed profiles give clues about outflows, infall motions, or rotation (Zhou 1992; Myers et al. 1996; Evans 1999; Narayanan et al. 2002; Sun \& Gao 2009). Infalls are usually accompanied by outflows due to accretion during the early stages of protostar formation (Li et al. 2014) and can be well traced by enhanced abundance of SiO (Schilke et al. 1997). Strong red profiles are found as outflow signs in a survey of extended green objects (EGOs) in massive star-forming regions (Chen et al. 2010). In order to find possible infall or outflow candidate sources, generally optically thick lines are used based on their doublepeaked and strong blue or red peak profiles. On the other hand, optically thin lines, for example of $\mathrm{H}^{13} \mathrm{CO}^{+}$, will not give a double-peaked profile and can be used to determine the systemic velocities of the clumps. In cases with double or multiple line profiles in optically thin lines, we assumed multiple cores along the line of sight. We picked these sources as outflow candidates in cases of a strong red profile and line wings.

In Sect. 2, we briefly introduce the selected sample and the ATLASGAL, MALT90, and Spitzer data used in this study. In Sect. 3, we present the spectral line analysis, and derived physical parameters. In Sect. 4, we discuss the kinematics, abundance ratios of different molecular species, and their relations in order to understand the evolutionary trends in chemical compositions. Finally, in Sect. 5 we summarize our results.

\footnotetext{
2 The Mopra radio telescope is part of the Australia Telescope National Facility which is funded by the Commonwealth of Australia for operation as a National Facility managed by CSIRO.
}

\section{Data}

\subsection{Archival data}

In this study, we have used the ATLASGAL data by downloading the fits files from the online $\operatorname{archive}^{3}$ in order to visually inspect the dust emission maps and compare them to the MALT90 molecular line emission maps. The resolution with the Large APEX Bolometer Camera (LABOCA) at $870 \mu \mathrm{m}$ wavelength is 19.' 2 FWHM which makes the ATLASGAL Survey observations sensitive to $\sim 0.5 \mathrm{pc}$ molecular clumps at a distance of $5 \mathrm{kpc}$. As a starting point, we selected all the clumps identified by ATLASGAL as dark in the $8 / 24 \mu \mathrm{m}$ bands and retrieved their information such as $V_{\mathrm{LSR}}$, mass, distance, and radius from Giannetti et al. (2014) and Wienen et al. (2015). Since the dust emission is generally optically thin in the submm band, the masses of the clumps are proportional to the total flux densities. However, there might be large uncertainty in the mass values due to uncertainties in the dust temperature as stated in Motte et al. (2007). Distances of the ATLASGAL clumps are taken from Wienen et al. (2015) where they used HI self-absorption and HI absorption methods to resolve the kinematic distance ambiguity.

We downloaded the MALT90 data cubes of selected targets from the online archive ${ }^{4}$. The MALT90 Survey mapped the $90 \mathrm{GHz}$ line emission with an angular resolution of $38^{\prime \prime}$ and spectral resolution of $0.11 \mathrm{~km} \mathrm{~s}^{-1}$. It provides simultaneously obtained 16 molecular emission lines that are typically observed in dense molecular clumps and cores. We used molecular lines of $\mathrm{HCO}^{+}$, $\mathrm{H}^{13} \mathrm{CO}^{+}, \mathrm{HNC}, \mathrm{HCN}$, and $\mathrm{N}_{2} \mathrm{H}^{+}$since they are the strongest species with their high critical densities. We also analyzed isotopologues of these lines, which are used to calculate the optical depths and thus column densities. $\mathrm{SiO}$ data is used to investigate possible outflows. The Spitzer/IRAC $3.6 \mu \mathrm{m}, 4.5 \mu \mathrm{m}, 5.8 \mu \mathrm{m}$, and $8 \mu \mathrm{m}$ mid-IR images and MIPS $24 \mu \mathrm{m}$ images from MIPSGAL were also used to create three-color images in order to visually inspect the infrared appearance of the environment of the clumps.

\subsection{Sample selection}

The first stage was to select the sources which were previously identified in the ATLASGAL catalog as dark clumps at $8 / 24 \mu \mathrm{m}$ wavelengths (from Table A.1 in Giannetti et al. 2014) which were 45 in total. Giannetti et al. (2014) identified a source as $8 \mu \mathrm{m}$ or $24 \mu \mathrm{m}$-dark if its flux density is smaller than the average flux at the same wavelength in the vicinity of the source. Following that, we downloaded the molecular line data of 36 high-mass clumps which were observed with MALT90 survey. Such observations of thirty of these sources (with high signal-to-noise ratios) were analyzed in this study. Table 1 summarizes the physical properties of these clumps taken from the ATLASGAL catalogs (Urquhart et al. 2018; Giannetti et al. 2014; Wienen et al. 2015).

\subsection{Molecular line data}

The MALT90 Survey observed 16 molecular lines at $90 \mathrm{GHz}$ toward $\sim 2000$ dense molecular clumps that were identified by the ATLASGAL Survey. Among these lines, $\mathrm{HCO}^{+}(1-0), \mathrm{HNC}(1-0)$, $\mathrm{HCN}(1-0)$, and $\mathrm{N}_{2} \mathrm{H}^{+}(1-0)$ are the brightest ones, and are density tracers. They are detected toward all clumps and analyzed in this study for the selected targets. In addition to $\mathrm{HCO}^{+}, \mathrm{N}_{2} \mathrm{H}^{+}, \mathrm{HCN}$, $\mathrm{HNC}$, and $\mathrm{SiO}$, the 1-0 lines of the isotopologues $\mathrm{H}^{13} \mathrm{CO}^{+}$and $\mathrm{HN}^{13} \mathrm{C}$, as density tracers, are also analyzed. Besides the density

\footnotetext{
3 http://atlasgal.mpifr-bonn.mpg.de/cgi-bin/ATLASGAL_ DATABASE.cgi

4 http://malt90.bu.edu and http://atoa.atnf.csiro.au/
} 
Table 1. Source table.

\begin{tabular}{|c|c|c|c|c|c|c|c|c|c|}
\hline No & Source $^{a}$ & RA (J2000) & $\operatorname{Dec}(\mathrm{J} 2000)$ & $\begin{array}{l}V_{\mathrm{LSR}} \\
\left(\mathrm{km} \mathrm{s}^{-1}\right)\end{array}$ & $\begin{array}{l}\text { Distance } \\
(\mathrm{kpc})\end{array}$ & $\begin{array}{l}\text { Mass } \\
\left(10^{3} M_{\odot}\right)\end{array}$ & $\begin{array}{l}\text { Radius } \\
\text { (pc) }\end{array}$ & Comments $^{b}$ & Comments $^{c}$ \\
\hline 1 & G008.684-00.367 & $18: 06: 23.35$ & $-21: 37: 05.2$ & 37.6 & 4.4 & 2.2 & 1.7 & D8 & MSF \\
\hline 2 & G008.706-00.414 & $18: 06: 36.72$ & $-21: 37: 18.6$ & 38.5 & 11.8 & 5.3 & 2.9 & D24 & YSO \\
\hline 3 & G010.444-00.017 & $18: 08: 44.59$ & $-19: 54: 36.4$ & 72.4 & 10.2 & 4.3 & 2.3 & D24 & MSF \\
\hline 4 & $\mathrm{G} 013.178+00.059$ & $18: 14: 01.32$ & $-17: 28: 38.6$ & 48.5 & 4.5 & 1.0 & 1.6 & D8 & MSF \\
\hline 5 & G014.114-00.574 & $18: 18: 13.14$ & $-16: 57: 20.0$ & 20.1 & 1.9 & 0.8 & 1.3 & D8 & YSO \\
\hline 6 & G014.194-00.194 & $18: 16: 58.77$ & $-16: 42: 17.5$ & 39.4 & 3.1 & 2.7 & 1.4 & D8 & YSO \\
\hline 7 & G014.492-00.139 & $18: 17: 22.09$ & $-16: 24: 59.4$ & 39.7 & 3.2 & 2.0 & 2.7 & D24 & YSO \\
\hline 8 & G014.632-00.577 & $18: 19: 15.22$ & $-16: 30: 02.3$ & 18.2 & 1.9 & 0.5 & 1.0 & D8 & MSF \\
\hline 9 & G018.876-00.489 & $18: 27: 07.69$ & $-12: 41: 36.4$ & 65.5 & 5.0 & 1.2 & 1.5 & D8 & YSO \\
\hline 10 & G309.382-00.134 & $13: 47: 24.03$ & $-62: 18: 09.5$ & -49.5 & 6.7 & 2.4 & 3.2 & D8 & MSF \\
\hline 11 & G317.867-00.151 & $14: 53: 16.60$ & $-59: 26: 33.9$ & -40.2 & 2.5 & 0.3 & 0.7 & D8 & YSO \\
\hline 12 & G318.779-00.137 & $14: 59: 33.06$ & $-59: 00: 33.5$ & -37.5 & 10.2 & 13.5 & 5.2 & D8 & YSO \\
\hline 13 & G320.881-00.397 & $15: 14: 33.18$ & $-58: 11: 28.5$ & --44.8 & 2.8 & 0.4 & 0.7 & D24 & YSO \\
\hline 14 & G326.987-00.031 & $15: 49: 08.16$ & $-54: 23: 06.9$ & -58.2 & 3.5 & 1.0 & 1.1 & D8 & Protostellar \\
\hline 15 & G329.029-00.206 & $16: 00: 31.96$ & $-53: 12: 54.0$ & -43.5 & 2.8 & 2.8 & 1.9 & D8 & MSF \\
\hline 16 & G331.708+00.583 & $16: 10: 06.79$ & $-50: 50: 29.4$ & -66.8 & 10.8 & 12.1 & 4.7 & D8 & YSO \\
\hline 17 & G333.656+00.059 & $16: 21: 11.61$ & $-49: 52: 16.3$ & -84.2 & 5.0 & 2.7 & 3.1 & D24 & Protostellar \\
\hline 18 & G335.789+00.174 & $16: 29: 47.34$ & $-48: 15: 52.1$ & -49.5 & 3.3 & 1.9 & 2.2 & D8 & MSF \\
\hline 19 & G336.958-00.224 & $16: 36: 17.12$ & $-47: 40: 44.3$ & -71.1 & 10.9 & 1.5 & 1.8 & D24 & MSF \\
\hline 20 & G337.176-00.032 & $16: 36: 18.78$ & $-47: 23: 18.6$ & -65.6 & 11.2 & 23.9 & 8.7 & D8 & MSF \\
\hline 21 & G337.258-00.101 & $16: 36: 56.42$ & $-47: 22: 27.0$ & -67.6 & 11.1 & 10.5 & 5.4 & D8 & MSF \\
\hline 22 & $\mathrm{G} 338.786+00.476$ & $16: 40: 22.55$ & $-45: 51: 04.1$ & -64.6 & 11.3 & 2.2 & 2.6 & D24 & Protostellar \\
\hline 23 & G340.784-00.097 & $16: 50: 14.89$ & $-44: 42: 31.6$ & -101.4 & 9.6 & 3.7 & 3.5 & D8 & MSF \\
\hline 24 & G342.484+00.182 & $16: 55: 02.53$ & $-43: 13: 03.1$ & -41.3 & 12.6 & 11.0 & 6.1 & D8 & MSF \\
\hline 25 & G343.756-00.164 & $17: 00: 50.08$ & $-42: 26: 11.9$ & -26.9 & 2.5 & 1.3 & 1.5 & D8 & MSF \\
\hline 26 & G351.444+00.659 & $17: 20: 54.60$ & $-35: 45: 12.8$ & -3.9 & 1.4 & 3.1 & 1.0 & D8 & MSF \\
\hline 27 & G351.571+00.762 & $17: 20: 51.00$ & $-35: 35: 25.8$ & -2.7 & 1.3 & 2.0 & 1.3 & D24 & YSO \\
\hline 28 & G353.066+00.452 & $17: 26: 13.50$ & $-34: 31: 54.0$ & 1.9 & 1.3 & 0.2 & 0.7 & D24 & YSO \\
\hline 29 & G353.417-00.079 & $17: 29: 18.94$ & $-34: 32: 05.9$ & -54.2 & 10.2 & 6.8 & 3.1 & D24 & Protostellar \\
\hline 30 & G354.944-00.537 & $17: 35: 11.69$ & $-33: 30: 23.8$ & -5.5 & 1.4 & 0.1 & 0.6 & D24 & YSO \\
\hline
\end{tabular}

Notes. Kinematic distances, $V_{\mathrm{LSR}}$, mass and radius are taken from Urquhart et al. (2018) which provides the most updated values based on previous ATLASGAL studies and follow-up studies (e.g., Wienen et al. 2015; Giannetti et al. 2014). Urquhart et al. (2018) combined the solutions from HI self-absorption and HI absorption methods using molecular line observations, maser parallax and spectroscopic distance measurements when available. Masses are based on $870 \mu \mathrm{m}$ dust continuum and $\mathrm{NH}_{3}$ lines. There might be large uncertainty in the mass due to uncertain dust emissivity as stated in Motte et al. (2007). Also, note that, the clumps are extended compared to the beam size, thus the mass values are above the sensitivity limit of ATLASGAL. ${ }^{(a)}$ Massive clumps that were detected by the ATLASGAL survey (Giannetti et al. 2014) and classified as objects dark at $8.0 \mu \mathrm{m}$ or $24.0 \mu \mathrm{m} .{ }^{(b)}$ Giannetti et al. (2014) identified the sources as dark at $8.0 \mu \mathrm{m}$ or $24.0 \mu \mathrm{m}$ if the flux density is smaller than the average flux at the same wavelength in the vicinity of the source. These sources are marked as D8 or D24. ${ }^{(c)}$ Urquhart et al. (2018) provides associated sources such as massive star forming (MSF) regions and young stellar objects (YSO) from the follow-up observations of these clumps.

tracers, another important molecular line observed in star-forming regions where shocks/outflows are present is $\mathrm{SiO}(2-1)$. There are multiple processes that can produce $\mathrm{SiO}$ emission in molecular clumps which can be a good tracer for both medium-velocity shocks $\left(\sim 10-20 \mathrm{~km} \mathrm{~s}^{-1}\right)$ or high-velocity shocks $\left(\sim 20-50 \mathrm{~km} \mathrm{~s}^{-1}\right)$ as discussed in Miettinen (2014) and references therein. The line data used in this study are shown in Table 2.

\section{Analysis}

\subsection{Spectral line fitting}

The CLASS program of the GILDAS software package ${ }^{5}$ was used to analyze the spectra. First, the spectra for each line were averaged and checked for single- or double-peaked profiles

\footnotetext{
5 Grenoble Image and Line Data Analysis Software is provided and actively developed by IRAM and is available at http://www.iram. fr/IRAMFR/GILDAS
}

(e.g., Fig. 1) and the first fit was applied. Spectral lines along with their isotopologues were averaged from a $38^{\prime \prime} \times 38^{\prime \prime}$ square region (the size of the beam) centered on the peak emission from $\mathrm{N}_{2} \mathrm{H}^{+}(1-0)$ and the initial guesses from the first fit were used to fit the spectra in the boxed region. The $\mathrm{HCO}^{+}, \mathrm{H}^{13} \mathrm{CO}^{+}, \mathrm{SiO}$, $\mathrm{HNC}$, and $\mathrm{HN}^{13} \mathrm{C}$ lines were fit with a single or double Gaussian profile since they do not have hyperfine line structures. Most of the sources have multiple line profiles which might be due to line-of-sight confusion, multiple cores, infall, or outflow. If there were double peaked profiles in the $\mathrm{HCO}^{+}$or $\mathrm{HNC}$ lines, they were fitted with two velocity components and then the profiles of optically thin lines $\left(\mathrm{H}^{13} \mathrm{CO}^{+}\right.$and/or $\left.\mathrm{HN}^{13} \mathrm{C}\right)$ were checked in order to examine the origin of the observed line profile. If optically thin lines showed double peaked profiles, it was considered that multiple cores were being sampled along the line of sight, otherwise infall, or outflow possibilities were considered. Within the target group only one source (G335.790+00.174) had double peaked shapes in its optically thin lines. 

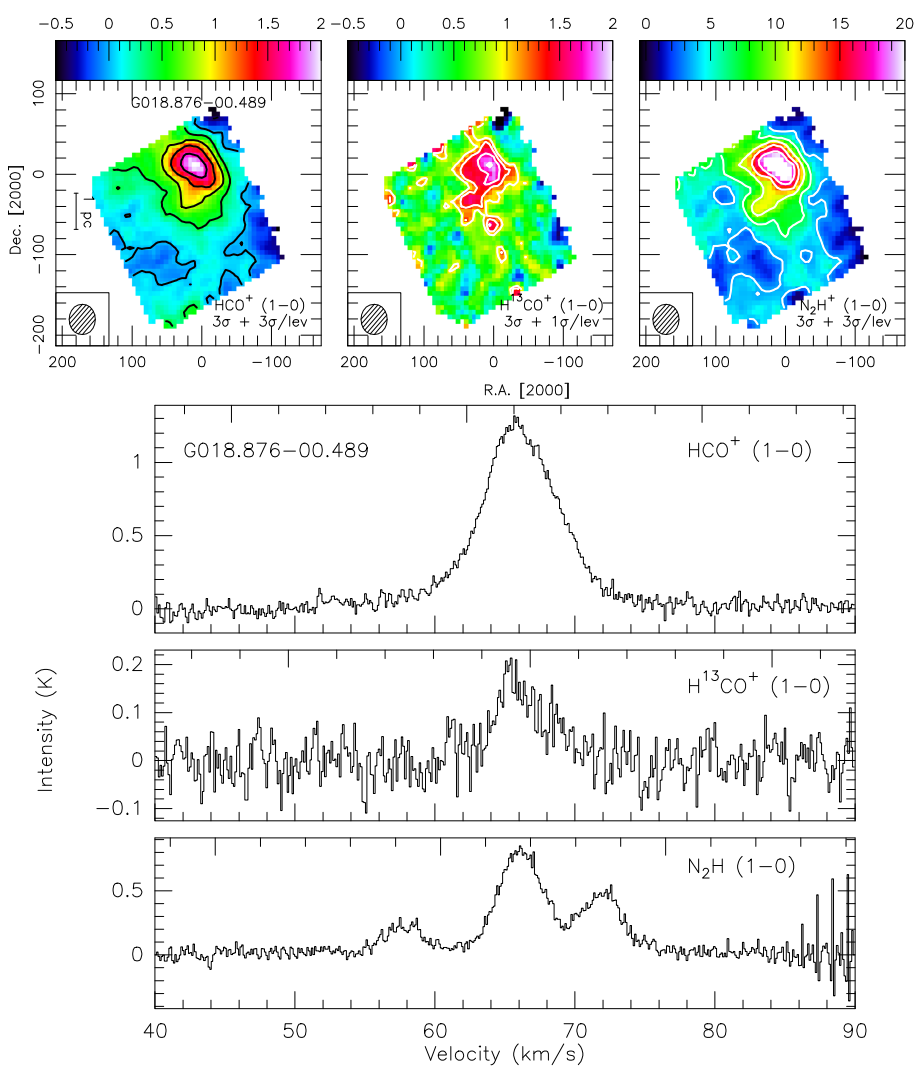

Fig. 1. Top panels: integrated intensity map of the $\mathrm{HCO}^{+}, \mathrm{H}^{13} \mathrm{CO}^{+}$, and $\mathrm{N}_{2} \mathrm{H}^{+}$emission for the G018.876-00.489 clump. Bottom panels: observed averaged spectra over the whole map for $\mathrm{HCO}^{+}, \mathrm{H}^{13} \mathrm{CO}^{+}$, and $\mathrm{N}_{2} \mathrm{H}^{+}$.

Table 2. MALT90 Molecular line observation parameters.

\begin{tabular}{lcc}
\hline \hline Lines & $\begin{array}{c}\text { Frequency } \\
(\mathrm{MHz})\end{array}$ & Tracer \\
\hline $\mathrm{HCO}^{+}(1-0)^{b}$ & 89188.526 & Density, kinematics \\
$\mathrm{N}_{2} \mathrm{H}^{+}(1-0)^{c}$ & 93173.770 & Density, chemically robust \\
$\mathrm{HNC}(1-0)^{b}$ & 90663.572 & Density, cold chemistry \\
$\mathrm{HCN}(1-0)^{d}$ & 88631.847 & Density \\
$\mathrm{H}^{13} \mathrm{CO}^{+}(1-0)$ & 86754.330 & Optical depth, column density, $V_{\mathrm{LSR}}$ \\
$\mathrm{HN}^{13} \mathrm{C}(1-0)$ & 87090.859 & Optical depth, column density, $V_{\mathrm{LSR}}$ \\
$\mathrm{SiO}(2-1)$ & 86847.010 & Shock, outflow \\
\hline
\end{tabular}

Notes. ${ }^{(a)}$ The frequencies for the lines were obtained from the Cologne Database for Molecular Spectroscopy (CDMS, Müller et al. 2001, 2005) and the Leiden atomic and molecular database (LAMDA; Schöier et al. 2005). ${ }^{(b)}$ Some of the $\mathrm{HCO}^{+}$and $\mathrm{HNC}$ lines show double peaks due to infall, outflow, rotation, etc. ${ }^{(c)} \mathrm{N}_{2} \mathrm{H}^{+}$line has 15 hyperfine components. ${ }^{(d)} \mathrm{HCN}$ line has three hyperfine components.

Integrated intensities were measured over a velocity range that was decided based on each line profile or line width. In Fig. A.1, the color scale and contours show the integrated intensity maps, and the spectra are the average spectra for each line.

For a single transition CLASS provides four line parameters: the integrated intensity, $\mathrm{W}\left(\mathrm{K} \mathrm{km} \mathrm{s}^{-1}\right)$, the local standard of rest velocity, $V_{\mathrm{LSR}}\left(\mathrm{km} \mathrm{s}^{-1}\right)$, FWHM line width, $\Delta V\left(\mathrm{~km} \mathrm{~s}^{-1}\right)$, and the main beam temperature, $T_{\mathrm{mb}}(\mathrm{K})$. On the other hand, $\mathrm{N}_{2} \mathrm{H}^{+}$and HCN were fit with the hyperfine structure (HFS) method to derive $A \times \tau_{\mathrm{m}}, V_{\mathrm{LSR}}, \Delta V$, and $\tau$, the sum of all the opacities of all hyperfine transitions. The $\mathrm{N}_{2} \mathrm{H}^{+}$line has 15 hyperfine components, while the HCN line has three which makes it possible to calculate the optical depths since optical depth can be written as a function of the relative strengths of the hyperfine components. The line parameters from the Gaussian fits are given in Table B. 1 and $\mathrm{N}_{2} \mathrm{H}^{+}$and $\mathrm{HCN}$ line parameters are given in Table B.2. Intensity (W) for $\mathrm{N}_{2} \mathrm{H}^{+}$, and $\mathrm{HCN}$ were derived by integrating the line over a wavelength range that covers all hyperfine line components $\left(20.5 \mathrm{~km} \mathrm{~s}^{-1}\right.$ on average for all sources). In order to estimate the uncertainty of the integrated intensity, we first derived the channel-by-channel rms noise by fitting the baseline with the line-free channels, then we followed Eq. (3) in Greve et al. (2009) taking into account the number of channels of the integrated intensity and baseline fitting. Observed spectra averaged from a $38^{\prime \prime} \times 38^{\prime \prime}$ square region are shown for all sources along with the integrated intensity maps in Fig. A.1. We note that for almost half of the sources, a good fit to the HCN spectrum was not possible due to the existing hyperfine line anomalies. As discussed in Loughnane et al. (2012) hyperfine anomalies in the $J=1 \rightarrow 0$ line are observed especially in line widths as well as the line strengths in massive star-forming regions.

\subsection{Optical depths and excitation temperatures}

In order to calculate the column densities, we first need to calculate the optical depths and excitation temperatures. Following the hyperfine line fitting for the $\mathrm{N}_{2} \mathrm{H}^{+}$line, $A \times \tau_{\mathrm{m}}$ and $\tau$ were derived. By using these parameters, and Eq. (1) below (as described in Sánchez-Monge 2011), excitation temperature was estimated.

$T_{\mathrm{ex}}=\frac{h v / k}{\ln \left[\frac{h v / k}{\left(A \tau_{\mathrm{m}} / \tau\right)+J_{v}\left(T_{\mathrm{bg}}\right)}+1\right]}$,

where $h$ is the Planck constant, $k$ is the Boltzmann constant, $v$ is the frequency of the observed transition, and $J_{v}\left(T_{\mathrm{bg}}\right)$ is the Planck radiation which was calculated by the following equation

$J_{v}\left(T_{\mathrm{bg}}\right)=\frac{h v / k}{e^{h v / k T_{\mathrm{bg}}}-1}$,

where the background temperature, $T_{\mathrm{bg}}$, is assumed to be $2.74 \mathrm{~K}$.

$T_{\text {ex }}$ was used for the rest of the measurements if the error was smaller than 30\% (as in Fontani et al. 2011), otherwise $11 \mathrm{~K}$ and $22 \mathrm{~K}$ was used for D24 and D8 clumps, respectively, which were calculated as average $T_{\mathrm{ex}}$ in Giannetti et al. (2014). For the $\mathrm{HCO}^{+}, \mathrm{H}^{13} \mathrm{CO}^{+}, \mathrm{SiO}, \mathrm{HNC}, \mathrm{HN}^{13} \mathrm{C}$, and $\mathrm{HCN}$ lines, we assumed they share the same $T_{\text {ex }}$ as $\mathrm{N}_{2} \mathrm{H}^{+}$.

CLASS provides the optical depths for the lines that have hyperfine structure since there is a constant ratio between the hyperfine lines, assuming local thermodynamic equilibrium (LTE). While we provide the optical depths for the $\mathrm{N}_{2} \mathrm{H}^{+}$line in Table B.2, we could not calculate the optical depths for half of the sources with the HCN line. In many such cases, we could not provide a good fit due to hyperfine line anomalies as mentioned in Sect. 3.1. The mean optical depths are $1.40 \pm 0.09$, and $3.64 \pm 0.28$ for the $\mathrm{N}_{2} \mathrm{H}^{+}$and $\mathrm{HCN}$ lines, respectively.

We derived the optical depths for lines of $\mathrm{HCO}^{+}$and $\mathrm{HNC}$ and their isotopologues $\mathrm{H}^{13} \mathrm{CO}^{+}$, and $\mathrm{HN}^{13} \mathrm{C}$ by following the method described in detail in Sanhueza et al. (2012). This method requires the main beam brightness temperature for both the main and the isotopologue lines and assumes a constant abundance ratio of 50 for $\left[\mathrm{HCO}^{+} / \mathrm{H}^{13} \mathrm{CO}^{+}\right]$and $\left[\mathrm{HNC} / \mathrm{HN}^{13} \mathrm{C}\right]$ (e.g., Savage et al. 2002). For one source (G014.114-00.574) 
since the main beam brightness temperature for the isotopologue was larger than the main molecule $\left(T_{\mathrm{H}^{13} \mathrm{CO}^{+}}>T_{\mathrm{HCO}^{+}}\right)$possibly due to self absorption, we could not calculate the optical depths. For three other sources we could not fit $\mathrm{H}^{13} \mathrm{CO}^{+}$data so we do not provide optical depths for those sources as well. For the same reasons for nine sources we could not calculate the optical depths for the $\mathrm{HNC}$ and $\mathrm{HN}^{13} \mathrm{C}$ lines. Optical depths are reported in Table B. 3 and the mean values are 20.3 and 23.2 for the optically thick $\mathrm{HCO}^{+}$and $\mathrm{HNC}$ lines, respectively, and 0.4 and 0.4 for the optically thin isotopologues $\mathrm{H}^{13} \mathrm{CO}^{+}$and $\mathrm{HN}^{13} \mathrm{C}$, respectively.

\subsection{Column densities}

Following the calculations of $T_{\mathrm{ex}}$ and the optical depths, the column densities were estimated for all the lines under the assumption of LTE. The following equation (Caselli et al. 2002) was used to calculate the column densities for optically thin lines, $N_{\text {thin }}$ :

$N_{\text {thin }}=\frac{8 \pi W}{\lambda^{3} A} \frac{g_{1}}{g_{\mathrm{u}}} \frac{1}{J_{v}\left(T_{\mathrm{ex}}\right)-J_{v}\left(T_{\mathrm{bg}}\right)} \frac{1}{1-e^{\left(-h v / k T_{\mathrm{ex}}\right)}} \frac{Q_{\mathrm{rot}}}{g_{1} e^{\left(-E_{1} / k T_{\mathrm{ex}}\right)}}$,

where $W$ is the integrated intensity of the line calculated from fitting single line transitions (see Table B.1), while $\mathrm{g}_{\mathrm{l}}$ and $\mathrm{g}_{\mathrm{u}}$ are the statistical weights of the lower and upper levels, $A$ is the Einstein coefficient, $Q_{\text {rot }}$ is the partition function, and $E_{1}$ is the lower energy level. Cataloged terms mentioned above are obtained for each line from the CDMS (The Cologne Database for Molecular Spectroscopy) and JPL (Jet Propulsion Laboratory, Molecular Spectroscopy) databases.

When the line is optically thick ( $\tau>1)$, the following equation was used to estimate the column density

$N_{\text {thick }}=N_{\text {thin }} \frac{\tau}{1-e^{-\tau}}$

For sources where a line is not detected, we calculated the upper limits by assuming optically thin lines with a line width of $2 \mathrm{~km} \mathrm{~s}^{-1}$, and a brightness temperature that is three times the rms of the spectra $\left(T_{\mathrm{mb}}=3 T_{\mathrm{rms}}\right)$. Estimated column densities are listed in Table B.4.

Mean, median, and standard deviation values of the column densities are listed at the end of the Table B.4. The derived column densities for $\mathrm{N}_{2} \mathrm{H}^{+}$range from $4.4 \times 10^{12} \mathrm{~cm}^{-2}$ to $116.2 \times 10^{12} \mathrm{~cm}^{-2}$ with an average value of $3.2( \pm 0.3) \times 10^{13} \mathrm{~cm}^{-2}$. This mean is similar to the value $\left(2.1 \times 10^{13} \mathrm{~cm}^{-2}\right)$ derived for a set of IRDCs by Sakai et al (2008) and smaller than the average value, $6.3 \times 10^{13} \mathrm{~cm}^{-2}$, derived for a set of massive young stellar objects and HII regions by Yu \& Wang (2015). This mean is also higher than the $10^{12} \mathrm{~cm}^{-2}$ which is a typical value found in low-mass cores (Caselli et al. 2002).

\section{Discussion}

\subsection{Infall and outflow candidates}

The low angular resolution of the single-dish data does not allow us to reveal the substructure of the clumps, but we can still for studying infall which is relevant to the high-mass star formation process. The infall candidates discussed in this study should be primary candidates to study the core structure of massive infrared clumps. For instance, they will give crucial information regarding the early phases of high-mass star formation, fragmentation and cluster formation processes when studied with radiative transfer modeling. As an example, Contreras et al. (2018) study the high-mass clump G331.372-00.116 with ALMA, which was detected by ATLASGAL and observed by MALT90 similarly to the high-mass clumps studied in this paper. By examining the $\mathrm{HCO}^{+}$and $\mathrm{HNC}$ lines and blue-shifted profiles, they estimate a core infall rate and discuss core collapse and competitive accretion scenarios. Within this context, one of the goals of our study is to find infall and outflow candidates among the dark clumps by examining their emission line morphology. For this purpose, we used the line profiles of $\mathrm{HCO}^{+}$, $\mathrm{H}^{13} \mathrm{CO}^{+}$, $\mathrm{HNC}$, and $\mathrm{HN}^{13} \mathrm{C}$. First, we examined the doublepeaked lines and if they have greater peak emission in their blue shifted sides, they were designated as infall candidates. G008.684-00.367, G008.706-00.414, G013.178+00.059, G014.114-00.574, G326.987-00.031， G329.029-00.206, G331.708+00.583_1, G331.708+00.583_2, G340.784-00.097, G343.756-00.164, G351.444+00.659, G353.066+00.452_2, and G354.944-00.537 have clear infall signatures based on the strong blue peaks in their $\mathrm{HCO}^{+}$lines. In order to determine the asymmetry of the line more quantitatively, we used the method given in Mardones et al. (1997) and determined the normalized velocity difference $(\delta v)$ between optically thick $\left(\mathrm{HCO}^{+}\right.$or $\left.\mathrm{HNC}\right)$ and thin $\left(\mathrm{H}^{13} \mathrm{CO}^{+}\right.$or $\left.\mathrm{HN}^{13} \mathrm{C}\right)$ components. The normalized velocity difference is given as

$\delta v=\frac{V_{\mathrm{LSR}}(\text { thick })-V_{\mathrm{LSR}}(\text { thin })}{\Delta V(\text { thin })}$.

If the $\mathrm{HCO}^{+}$or $\mathrm{HNC}$ lines have a significant blue shift $(\delta v<-0.25)$, the source was flagged as blue and if either of them has a significant red shift $(\delta v>0.25)$, the source was flagged as red as given in Table 3 . Thirteen of the sources have blue profiles (shown in Table 3), while seven of these sources had been previously found with blue profiles in He et al. (2015). Among these 13 sources, nine have blue profiles in both the $\mathrm{HCO}^{+}$and HNC lines.

In addition to the infall candidates with blue profiles, there are six sources with red profiles which are thought to be outflow indicators as stated in Chen et al. (2010). One of these sources, G014.632-00.577, has red profiles in both lines and it is shown in Fig. 3. In order to differentiate infall from outflow or rotation, the profile of the spectra should be investigated throughout the mapped region. Within this context, we examined the line profiles and overall the spectral map within each region. The spectral maps for the infall and outflow candidates are shown in Figs. 4 and 5 if they have high signal-tonoise ratio $(\mathrm{S} / \mathrm{N})$. For instance for previously mentioned outflow candidate $\mathrm{G} 14.632-00.577$, the $\mathrm{HCO}^{+}$spectra show a doublepeaked line profile at the central region of the clump, however, within the mapped area it changes from blue-shifted profile to the red-shifted profile as seen in Fig. 5. While this can be a sign for outflow, we note that rotation can also produce these kind of asymmetries. In this case, the velocity gradients of optically thin lines can be studied, however, insufficient $\mathrm{S} / \mathrm{N}$ with the current single-dish data do not allow us to do further investigation.

Among the targets, eleven sources have a significant $\mathrm{SiO}$ detection (over $3 \sigma$ ). Among them, G014.194-00.194, G014.632-00.575, G331.708+00.583, and G351.444+00.659 have excess in the line wings of $\mathrm{HCO}^{+}$spectra which is also a known effect of outflows (Rawlings et al. 2004).

Considering the high optical depths of the $\mathrm{HCO}^{+}$, and HNC lines, the lines should be highly affected by gradients in geometry, excitation temperature, and velocity fields along the clumps. Indeed, some studies show that the infall signatures may not be observable in all molecular tracers in some 
Table 3. List of the sources with blue and red profiles.

\begin{tabular}{|c|c|c|c|c|c|c|c|c|c|}
\hline $\begin{array}{l}\text { Source } \\
\left(\mathrm{km} \mathrm{s}^{-1}\right)\end{array}$ & $\begin{array}{l}V_{\mathrm{LSR}}\left(\mathrm{HCO}^{+}\right) \\
\left(\mathrm{km} \mathrm{s}^{-1}\right)\end{array}$ & $\begin{array}{l}V_{\mathrm{LSR}}\left(\mathrm{H}^{13} \mathrm{CO}^{+}\right) \\
\left(\mathrm{km} \mathrm{s}^{-1}\right)\end{array}$ & $\begin{array}{l}V_{\mathrm{LSR}}(\mathrm{HNC}) \\
\left(\mathrm{km} \mathrm{s}^{-1}\right)\end{array}$ & $\begin{array}{l}V_{\mathrm{LSR}}\left(\mathrm{HN}^{13} \mathrm{C}\right) \\
\left(\mathrm{km} \mathrm{s}^{-1}\right)\end{array}$ & $\begin{array}{l}\Delta V\left(\mathrm{H}^{13} \mathrm{CO}^{+}\right) \\
\left(\mathrm{km} \mathrm{s}^{-1}\right)\end{array}$ & $\begin{array}{l}\Delta V\left(\mathrm{HN}^{13} \mathrm{C}\right) \\
\left(\mathrm{km} \mathrm{s}^{-1}\right)\end{array}$ & $\begin{array}{l}\delta v\left(\mathrm{HCO}^{+}\right) \\
\left(\mathrm{km} \mathrm{s}^{-1}\right)\end{array}$ & $\delta v(\mathrm{HNC})$ & Profile \\
\hline G008.684-00.367 & $34.04 \pm 0.04$ & $36.94 \pm 0.10$ & $34.22 \pm 0.06$ & $37.40 \pm 0.12$ & $4.46 \pm 0.23$ & $5.30 \pm 0.40$ & $-0.65 \pm 0.04$ & $-0.60 \pm 0.05$ & $\mathrm{~B}, \mathrm{~B}^{b}$ \\
\hline G008.706-00.414 & $38.56 \pm 0.04$ & $39.09 \pm 0.12$ & $38.65 \pm 0.04$ & $39.38 \pm 0.11$ & $2.82 \pm 0.27$ & $1.81 \pm 0.22$ & $-0.19 \pm 0.05$ & $-0.41 \pm 0.08$ & $\mathrm{~N}, \mathrm{~B}^{b}$ \\
\hline G013.178+00.059 & $48.31 \pm 0.05$ & $49.06 \pm 0.17$ & $49.13 \pm 0.04$ & $49.44 \pm 0.15$ & $2.90 \pm 0.60$ & $3.70 \pm 0.40$ & $-0.25 \pm 0.08$ & $-0.09 \pm 0.04$ & $\mathrm{~B}, \mathrm{~N}^{b}$ \\
\hline G014.114-00.574 & $18.27 \pm 0.29$ & $19.96 \pm 0.12$ & $19.38 \pm 0.08$ & $20.90 \pm 0.21$ & $3.25 \pm 0.23$ & $3.50 \pm 0.50$ & $-0.52 \pm 0.10$ & $-0.44 \pm 0.09$ & $\mathrm{~B}, \mathrm{~B}$ \\
\hline G014.194-00.194 & $40.23 \pm 0.05$ & $39.52 \pm 0.10$ & $39.85 \pm 0.032$ & $39.47 \pm 0.14$ & $2.86 \pm 0.26$ & $2.80 \pm 0.40$ & $0.25 \pm 0.05$ & $0.14 \pm 0.05$ & $\mathrm{R}, \mathrm{N}$ \\
\hline G014.632-00.577 & $20.32 \pm 0.026$ & $18.79 \pm 0.08$ & $19.90 \pm 0.029$ & $18.81 \pm 0.07$ & $2.34 \pm 0.21$ & $1.94 \pm 0.16$ & $0.65 \pm 0.07$ & $0.56 \pm 0.06$ & $\mathrm{R}, \mathrm{R}$ \\
\hline G309.382-00.134 & $-49.06 \pm 0.05$ & $-50.38 \pm 0.22$ & $-50.37 \pm 0.07$ & - & $3.40 \pm 0.70$ & - & $0.39 \pm 0.10$ & - & $\mathrm{R}, \mathrm{N}$ \\
\hline G317.867-00.151 & $-38.87 \pm 0.09$ & $-40.07 \pm 0.21$ & $-38.92 \pm 0.29$ & - & $3.90 \pm 0.50$ & - & $0.30 \pm 0.07$ & - & $\mathrm{R}, \mathrm{N}$ \\
\hline G318.779-00.137 & $-36.45 \pm 0.08$ & $-38.59 \pm 0.25$ & $-37.87 \pm 0.09$ & $-37.94 \pm 0.34$ & $3.40 \pm 0.60$ & $3.90 \pm 0.70$ & $0.64 \pm 0.14$ & $0.02 \pm 0.09$ & $\mathrm{R}, \mathrm{N}$ \\
\hline G326.987-00.031 & $-59.91 \pm 0.05$ & $-58.39 \pm 0.27$ & $-59.72 \pm 0.19$ & $-58.46 \pm 0.22$ & $2.90 \pm 0.70$ & $2.30 \pm 0.50$ & $-0.53 \pm 0.16$ & $-0.54 \pm 0.18$ & $\mathrm{~B}, \mathrm{~B}$ \\
\hline G329.029-00.206 & $-46.47 \pm 0.04$ & $-43.30 \pm 0.16$ & $-46.31 \pm 0.05$ & $-43.21 \pm 0.11$ & $4.9 \pm 0.4$ & $3.33 \pm 0.29$ & $-0.64 \pm 0.06$ & $-0.93 \pm 0.09$ & $\mathrm{~B}, \mathrm{~B}$ \\
\hline G331.708+00.583_1 & $-69.33 \pm 0.06$ & $-67.20 \pm 0.21$ & $-68.75 \pm 0.10$ & $-67.05 \pm 0.20$ & $4.00 \pm 0.40$ & $3.40 \pm 0.50$ & $-0.53 \pm 0.08$ & $-0.49 \pm 0.10$ & $\mathrm{~B}, \mathrm{~B}^{b}$ \\
\hline G331.708+00.583_2 & $-69.57 \pm 0.034$ & $-67.14 \pm 0.14$ & $-68.95 \pm 0.030$ & $-67.17 \pm 0.14$ & $4.08 \pm 0.33$ & $2.63 \pm 0.29$ & $-0.59 \pm 0.06$ & $-0.68 \pm 0.09$ & $\mathrm{~B}, \mathrm{~B}^{b}$ \\
\hline G340.784-00.097 & $-102.46 \pm 0.14$ & $-100.87 \pm 0.10$ & $-101.55 \pm 0.06$ & $-101.16 \pm 0.25$ & $1.39 \pm 0.28$ & $2.70 \pm 1.70$ & $-1.14 \pm 0.27$ & $-0.14 \pm 0.13$ & $\mathrm{~B}, \mathrm{~N}$ \\
\hline $\mathrm{G} 342.484+00.182$ & $-40.92 \pm 0.07$ & $-41.53 \pm 0.08$ & $-41.04 \pm 0.012$ & - & $1.64 \pm 0.19$ & - & $0.38 \pm 0.08$ & - & $\mathrm{R}, \mathrm{N}$ \\
\hline G343.756-00.164 & $-29.87 \pm 0.07$ & $-27.69 \pm 0.08$ & $-29.12 \pm 0.034$ & $-27.60 \pm 0.12$ & $2.66 \pm 0.19$ & $2.92 \pm 0.33$ & $-0.82 \pm 0.07$ & $-0.52 \pm 0.07$ & $\mathrm{~B}, \mathrm{~B}$ \\
\hline G351.444+00.659 & $-5.68 \pm 0.02$ & $-4.35 \pm 0.031$ & $-4.46 \pm 0.014$ & $-4.31 \pm 0.04$ & $3.89 \pm 0.07$ & $3.89 \pm 0.10$ & $-0.34 \pm 0.011$ & $-0.04 \pm 0.01$ & $\mathrm{~B}, \mathrm{~N}^{b}$ \\
\hline G353.066+00.452_1 & $2.25 \pm 0.04$ & $1.62 \pm 0.14$ & $1.68 \pm 0.05$ & $1.56 \pm 0.26$ & $2.24 \pm 0.31$ & $2.60 \pm 0.60$ & $0.28 \pm 0.08$ & $0.05 \pm 0.10$ & $\mathrm{R}, \mathrm{N}$ \\
\hline G353.066+00.452_2 & $-2.89 \pm 0.03$ & $-1.80 \pm 0.08$ & $-2.25 \pm 0.04$ & $-1.68 \pm 0.22$ & $1.74 \pm 0.18$ & $1.60 \pm 0.40$ & $-0.63 \pm 0.08$ & $-0.36 \pm 0.17$ & $\mathrm{~B}, \mathrm{~B}$ \\
\hline G354.944-00.537 & $-6.85 \pm 0.07$ & $-5.90 \pm 0.11$ & $-6.54 \pm 0.05$ & $-6.14 \pm 0.23$ & $1.98 \pm 0.22$ & $1.08 \pm 0.40$ & $-0.48 \pm 0.09$ & $-0.22 \pm 0.14$ & $\mathrm{~B}, \mathrm{~B}^{b}$ \\
\hline
\end{tabular}

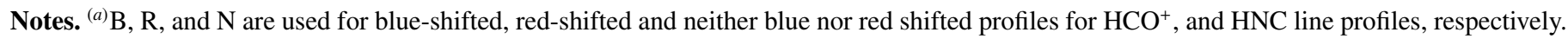
${ }^{(b)}$ These sources are found with either $\mathrm{HCO}^{+}$or $\mathrm{HNC}$ blue-shifted profiles in $\mathrm{He}$ et al. (2015) too.

cases. For example, Chira et al. (2014) examined the line profiles of $\mathrm{HCO}^{+}$and $\mathrm{HNC}$ for specific transitions by using radiative transfer calculations and showed that the infall signs seen in $\mathrm{HCO}^{+}$line profiles do not exist in HNC profiles in some transitions. Therefore, a line investigation with radiative transfer modeling for specific sources is needed to understand the detailed physics behind the lines.

\subsection{Mass infall rates}

In order to estimate the mass infall rate, we used $\mathrm{HCO}^{+}$and HNC molecular line emission of the sources with blue-peaked profiles. A radiative transfer model "Hill5" (De Vries \& Myers 2005) from the PySpecKit3 spectroscopic analysis toolkit (Ginsburg \& Mirocha 2011) allowed us to reproduce the observed line profiles and derive an infall velocity $\left(v_{\text {in }}\right)$ which can be used to estimate a clump mass infall rate $(\dot{M})$.

First, we fitted the average spectrum of $\mathrm{HCO}^{+}$and $\mathrm{HNC}$ for 13 infall candidates that are listed with blue profiles in Table 3. The Hill5 model uses the following free parameters for which you are required to provide initial guesses and also set limits: the optical depth of the center of the line $(\tau)$, the local standard of rest velocity $\left(V_{\mathrm{LSR}}\right)$, the infall velocity $\left(v_{\text {in }}\right)$, the velocity dispersion $(\sigma)$, and the peak excitation temperature $\left(T_{\text {peak }}\right)$. When the infall velocity is smaller than the velocity dispersion or the line profile does not show a separated red shifted peak, the fit results are not reliable. In these cases, we could not obtain a reliable fit. In the other cases the fits are shown in Fig. 2 and derived infall rates are listed in Table 4.

Secondly, we calculated the volume densities assuming spherical clumps by (Heyer et al. 2016)

$n\left(\mathrm{H}_{2}\right)=\frac{3 M_{\mathrm{cl}}}{4 \pi \mu m_{\mathrm{H}} R_{\mathrm{cl}}^{3}}$,

where $\mu=2.8, m_{\mathrm{H}}$ is the mass of the hydrogen atom, and $M_{\mathrm{cl}}$ and $R_{\mathrm{cl}}$ are the mass and radius of the clumps (from Table 1), respectively. By using this density value, and the infall velocity, $v_{\text {in }}$, from the "Hill5" model fit, we calculated a mass infall rate for each clump by

$\dot{M}=4 \pi R_{\mathrm{cl}}^{2} n\left(\mathrm{H}_{2}\right) \mu m_{\mathrm{H}} v_{\mathrm{in}}$.

Table 4 shows the volume densities and the mass infall rates for the ten infall candidates. Mass infall rates range between $0.2 \times 10^{-3} M_{\odot} \mathrm{yr}^{-1}-1.8 \times 10^{-2} M_{\odot} \mathrm{yr}^{-1}$ which are similar to the value, $1.3 \times 10^{-2} M_{\odot} \mathrm{yr}^{-1}$, found by Liu et al. (2013b) for the protocluster G10.6-0.4. We note that these infall rates refer to the gas accreted by the clump at larger scales, not at the smaller core scale. Previous mass infall rates found in high-mass protostellar regions range between $10^{-5}-10^{-3} M_{\odot} \mathrm{yr}^{-1}$ (e.g., Liu et al. 2017; Peretto et al. 2013) whose high end is similar to the values we found. These high infall rates indicate that the clumps are undergoing a global infall and feed the possible high-mass protostellar and dense cores, we cannot, however estimate how much gas is accreted on to the cores themselves with the current data.

\subsection{Nature of some of the infall candidates}

Some of the sources with infall and/or outflow signatures in MALT90 data were already studied by other authors. These studies confirmed them as infall and/or outflow candidates and revealed filamentary structures and multiple cores. This shows that MALT90 clumps are excellent targets to further study the early star formation process with higher quality data. Here we summarize the properties of three of these clumps while we will not give detailed discussion for all the clumps. In order to visually inspect the infrared appearance of the environment of these clumps, we generated the Spitzer images shown in Fig. 3. Spitzer images of the other clumps can be found in the ATLASGAL catalog 6 .

G008.684-00.367. An extended source is seen in the integrated intensity maps in the upper panels of Fig. A.1. The

\footnotetext{
6 https://atlasgal .mpifr-bonn.mpg.de/cgi-bin/ ATLASGAL_DATABASE.cgi
} 

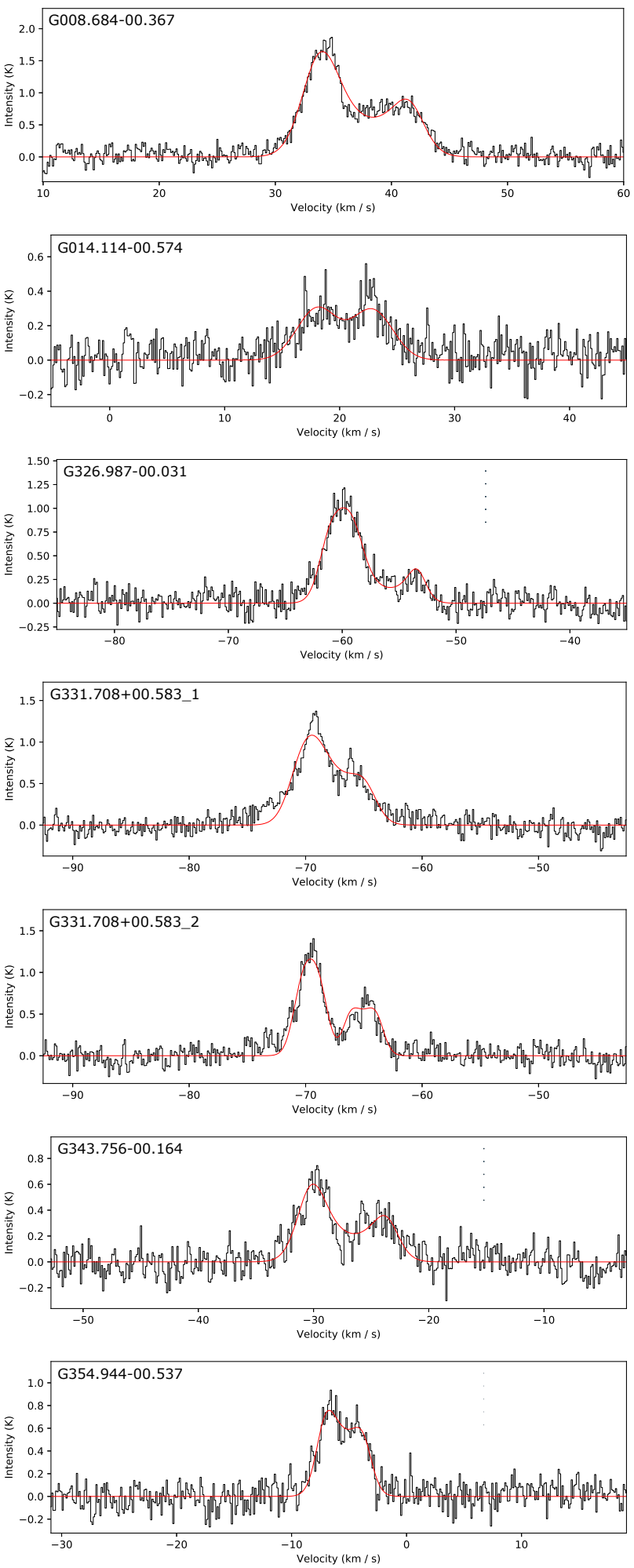

(a) $\mathrm{HCO}^{+}(1-0)$ spectrum
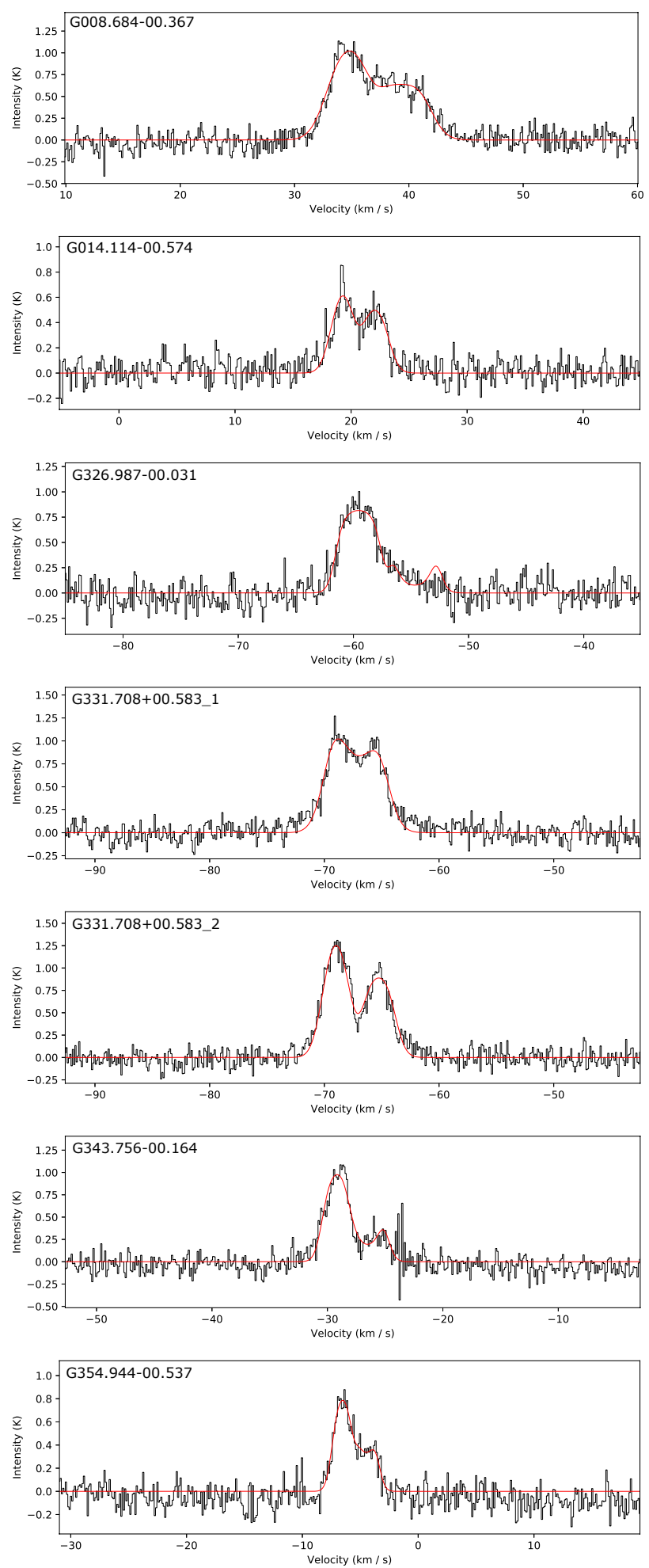

(b) HNC(1-0) spectrum

Fig. 2. Average $\mathrm{HCO}^{+}$(on left column) and $\mathrm{HNC}$ spectra (on right column) for the infall candidates are shown in black and the "Hills" fit in red.

$\mathrm{N}_{2} \mathrm{H}^{+}$emission shows a single elongated structure, where two cores can be seen in the $\mathrm{HN}^{13} \mathrm{C}$ integrated emission line map as well as in the dust continuum map. The bottom panels of Fig. A.1 show each spectral line. The $\mathrm{HCO}^{+}$and HNC lines have double peaked-profiles, while the $\mathrm{HN}^{13} \mathrm{C}$ line does not have a second component. Although the $\mathrm{H}^{13} \mathrm{CO}^{+}$line has a weak second component, it was not possible to obtain a good fit due to its low $\mathrm{S} / \mathrm{N}$. In order to examine the line profile change along the clump region, we generated the spectral maps as seen in Fig. 4. The topleft panel shows the double-peaked profile in the $\mathrm{HCO}^{+}$spectra in the clump region. A strong blue peaked profile in $\mathrm{HCO}^{+}$and $\mathrm{HNC}$ spectra is a typical infall signature (Table 3 ). 

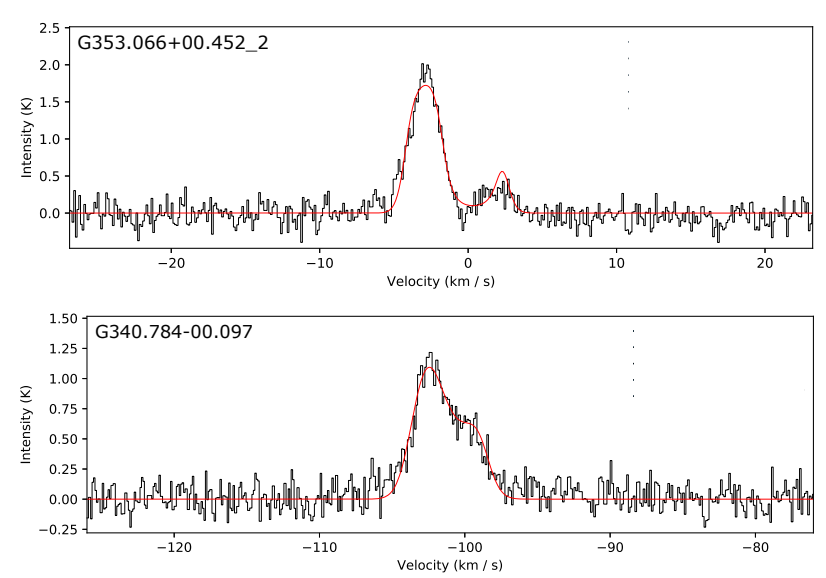

(a) $\mathrm{HCO}^{+}(1-0)$ spectra

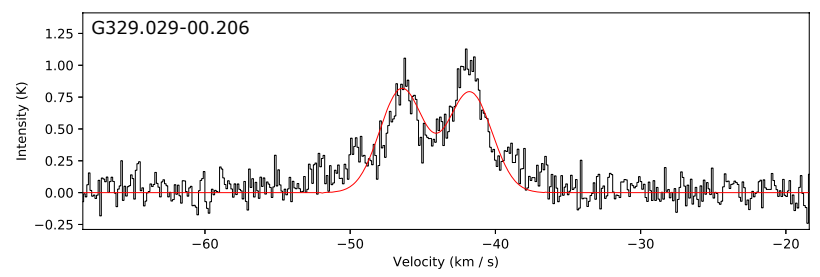

(b) $\mathrm{HNC}(1-0)$ spectra

Fig. 2. continued.

Table 4. Mass infall rates.

\begin{tabular}{lllllll}
\hline \hline No & Name & $\begin{array}{l}n\left(\mathrm{H}_{2}\right) \\
\left(10^{3} \mathrm{~cm}^{-3}\right)\end{array}$ & $\begin{array}{l}v_{\text {in }}\left(\mathrm{HCO}^{+}\right) \\
\left(\mathrm{km} \mathrm{s}^{-1}\right)\end{array}$ & $\begin{array}{l}v_{\text {in }}(\mathrm{HNC}) \\
\left(\mathrm{km} \mathrm{s}^{-1}\right)\end{array}$ & $\begin{array}{l}\dot{M}\left(\mathrm{HCO}^{+}\right) \\
\left(10^{-3} M_{\odot} \mathrm{yr}^{-1}\right)\end{array}$ & $\begin{array}{l}\dot{M}(\mathrm{HNC}) \\
\left(10^{-3} M_{\odot} \mathrm{yr}^{-1}\right)\end{array}$ \\
\hline 1 & G008.684-00.367 & 1.5 & $1.1 \pm 0.5$ & $2.35 \pm 0.49$ & $4.4 \pm 2.1$ & $9.32 \pm 1.94$ \\
2 & G014.114-00.574 & 1.3 & $2.0 \pm 1.0$ & $1.39 \pm 0.44$ & $3.8 \pm 1.9$ & $2.62 \pm 0.83$ \\
3 & G326.987-00.031 & 2.6 & $1.8 \pm 0.8$ & $2.43 \pm 0.52$ & $4.9 \pm 2.4$ & $6.77 \pm 1.45$ \\
4 & G329.029-00.206 & 1.4 & - & $2.34 \pm 0.35$ & - & $8.00 \pm 3.84$ \\
5 & G331.708+00.583_1 & 0.4 & $1.2 \pm 2.3$ & $0.23 \pm 0.75$ & $9.5 \pm 18.1$ & $1.81 \pm 5.92$ \\
6 & G331.708+00.583_2 & 0.4 & $2.3 \pm 0.3$ & $1.86 \pm 0.24$ & $17.8 \pm 2.5$ & $14.68 \pm 1.89$ \\
7 & G340.784-00.097 & 0.3 & $0.9 \pm 2.0$ & - & $3.0 \pm 6.5$ & - \\
8 & G343.756-00.164 & 1.3 & $0.7 \pm 1.2$ & $1.18 \pm 0.83$ & $2.0 \pm 3.2$ & $3.13 \pm 2.20$ \\
9 & G353.066+00.452_2 & 2.0 & $1.2 \pm 0.3$ & - & $1.1 \pm 0.2$ & - \\
10 & G354.944-00.537 & 1.6 & $1.2 \pm 0.4$ & $1.01 \pm 0.67$ & $0.2 \pm 0.5$ & $0.52 \pm 0.34$ \\
\hline
\end{tabular}

There is no infrared source detected at the central coordinates (see Fig. 3), and the low value of $T_{\mathrm{ex}}(5.11 \pm 0.25 \mathrm{~K})$ and high column density of $\mathrm{N}_{2} \mathrm{H}^{+}\left(55.8 \pm 9.7 \times 10^{12} \mathrm{~cm}^{-2}\right)$ indicate that the clump is at a very early stage of star formation. Extended $\mathrm{SiO}$ emission and high column density for $\mathrm{SiO}\left(1.4 \pm 0.2 \times 10^{12} \mathrm{~cm}^{-2}\right)$ indicate that there might be shocks in this clump due to outflows.

The clump might be part of the star-forming complex IRAS 18032-2137 where we see an embedded stellar cluster BDS2003-3 (Bica et al. 2003) and an UCHII region G8.67-0.36 (Wood \& Churchwell 1989). Longmore et al. (2011) studied this clump using SMA data and revealed three continuum sources separated by a minimum of $1.2^{\prime \prime}$ and $2.2^{\prime \prime}$ ( $\sim 6200 \mathrm{AU}$ and $\sim 9700 \mathrm{AU}$ ) and a bipolar outflow seems associated with one of the cores. Although the cores they studied have masses of $4 M_{\odot}, 7 M_{\odot}$, and $10 M_{\odot}$, Longmore et al. (2011) conclude that G008.684-00.367 can still form O-type stars through large-scale infall and accretion from the very massive gas reservoir surrounding it.

G014.114-00.574. An extended-filamentary source in all integrated intensity maps and dust continuum emission is shown in the upper panels of Fig. A.1. The $\mathrm{HCO}^{+}$and $\mathrm{HNC}$ lines show double-peaked profiles and blue profiles (see Table 3). It shows a small velocity gradient over $\sim 1 \mathrm{pc}$ in $\mathrm{N}_{2} \mathrm{H}^{+}$emission with $\sim 1 \mathrm{~km} \mathrm{~s}^{-1}$ as seen in Fig. 9. Filamentary structure, multiple cores in optically thin line emission maps, and the velocity gradient make this clump an interesting target for the study of early fragmentation in high-mass star formation.
The clump is located within the giant molecular cloud (GMC) M 17 SWex (Fig. 3), which extends $\sim 50 \mathrm{pc}$ southwest from the prominent Galactic HII region M $17(d=1.98 \mathrm{kpc}$, Wu et al. 2014), contains more than ten thousand YSO candidates, and represents a proto-OB association (Povich \& Whitney 2010). Povich et al. (2016) suggested that the whole GMC is a good example of a distributed mode of star formation which is dominated by intermediate-mass stars that will produce a large OB association with few massive clusters, or the massive cores will produce massive clusters since they are still accreting mass. Busquet et al. (2016) revealed 13 fragments within this clump (Hub-S), arranged in a manner that is more consistent with thermal Jeans fragmentation than a turbulent supported scenario.

G331.708+00.583. Two clumps are seen in all molecular emission lines as well as in dust continuum emission (Fig. A.1) and in $24 \mu \mathrm{m}$ infrared emission (Fig. 3). One is at the center, and the other is in the northwest corner. The central clump is associated with two EGOs which are in general thought to be tracing the jets/outflow from the MYSOs (Cyganowski et al. 2013).

The high column density of $\mathrm{N}_{2} \mathrm{H}^{+}$of each clump indicates that they are both at early stages, and the $\mathrm{N}_{2} \mathrm{H}^{+}$column density is higher in the second clump $\left(33.5 \pm 7.1 \times 10^{12} \mathrm{~cm}^{-2}\right)$ which might indicate that it is younger than the central clump, G331.708+00.583_1, $\left(16.1 \pm 2.5 \times 10^{12} \mathrm{~cm}^{-2}\right)$. The spectra for both clumps have multiple line components in optically thick lines and blue profiles in both the $\mathrm{HCO}^{+}$and $\mathrm{HNC}$ lines as seen in Fig. 4 and Table 3 which indicate that both clumps are possible infall targets. Furthermore, G331.708+00.583_1 shows line 
G. Saral et al.: MALT90 molecular content on high-mass IR-dark clumps
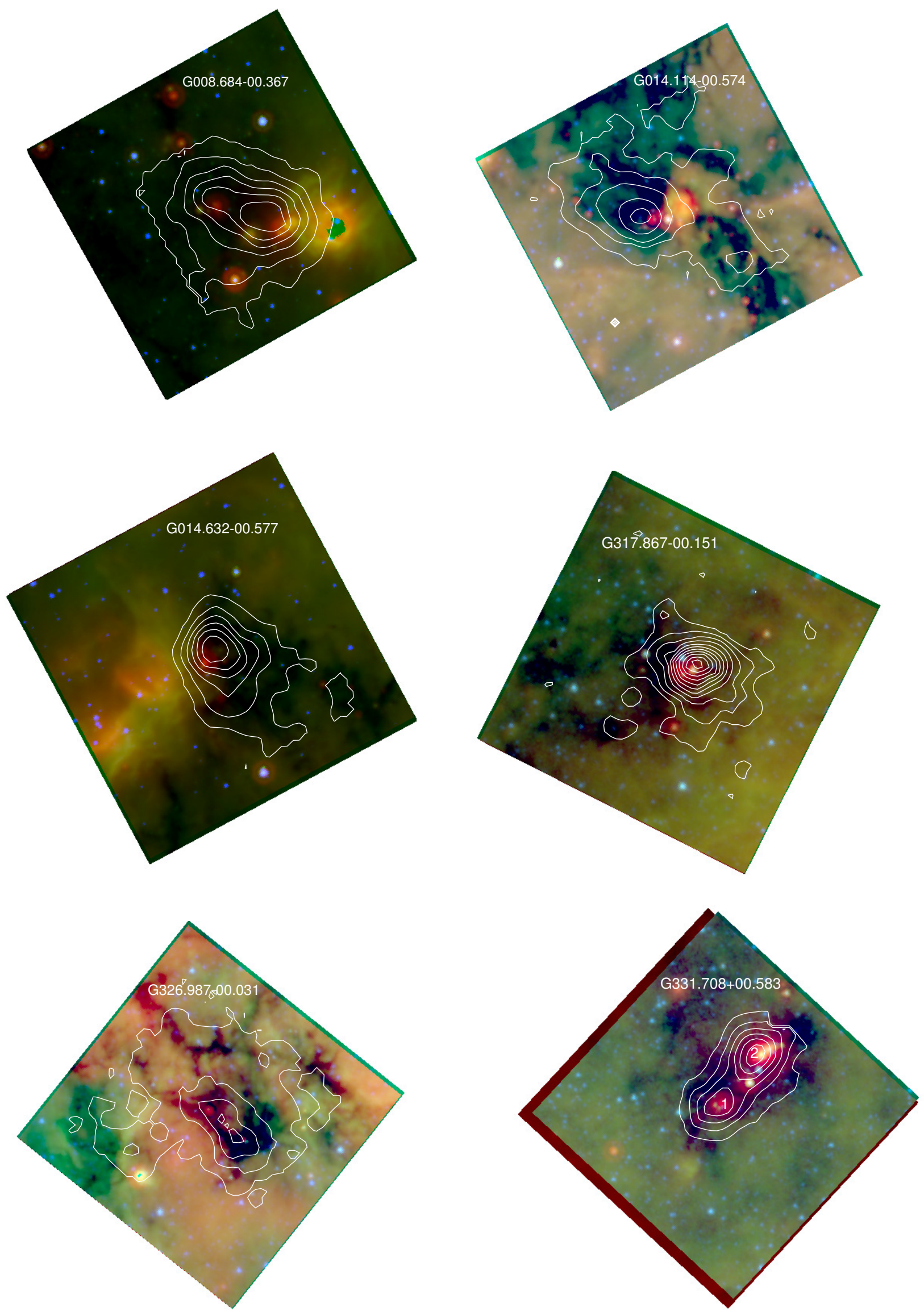

Fig. 3. Spitzer RGB images for some of the infrared dark clumps (blue: $3.6 \mu \mathrm{m}$, green: $8 \mu \mathrm{m}$, red: $24 \mu \mathrm{m}$ ) with $\mathrm{N}_{2} \mathrm{H}^{+}$emission overlaid with the contour levels of $3 \sigma, 6 \sigma, 9 \sigma$, etc. 

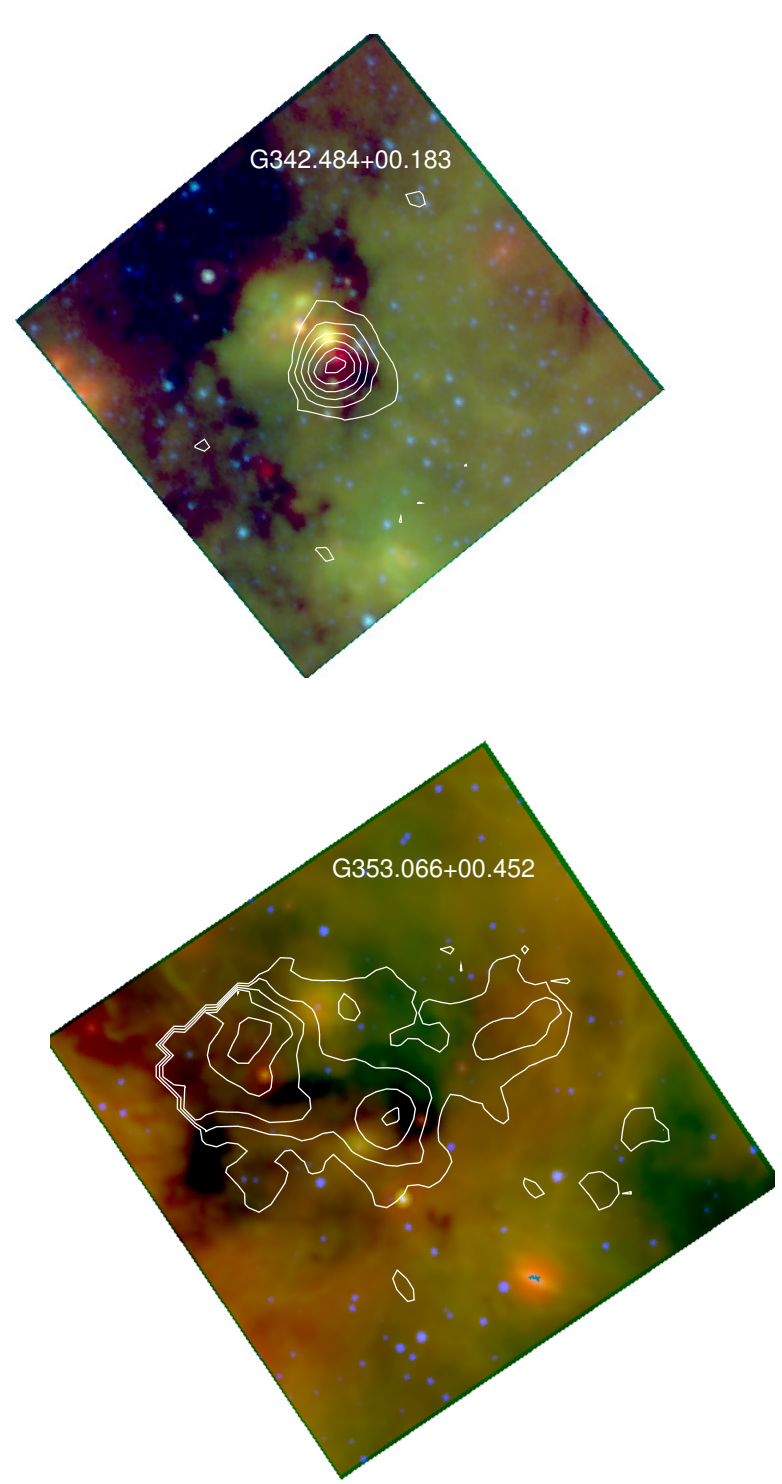

Fig. 3. continued.

wings in the $\mathrm{HCO}^{+}$and $\mathrm{HNC}$ lines which might be outflow indicators as shown in Cesaroni et al. (1997).

The infrared cloud that hosts both clumps is located in the fourth Galactic quadrant and the kinematic distance is either $4.0 \mathrm{kpc}$ or $10.7 \mathrm{kpc}$. Yu \& Wang (2015) also studied this source with MALT90 data, finding the blue profiles in the $\mathrm{HCO}^{+}$line indicating infall, as well as a red profile in the HNC line indicating an outflow activity. Both clumps are promising targets with which to investigate in detail the possible cores and outflows with high-sensitivity and high-resolution data.

\subsection{Kinematics}

In order to obtain information about the gas kinematics inside the molecular clumps, line widths can be used. Line broadening in optically thin lines is commonly observed in more active clumps due to increased turbulence with star formation activity as proposed by Chambers et al. (2009) and observed in some studies (e.g., Sakai et al. 2008; Vasyunina et al. 2011; Sanhueza et al. 2012). In order to see if there is any trend in the line widths, we first chose lines which are arguably optically thin, since
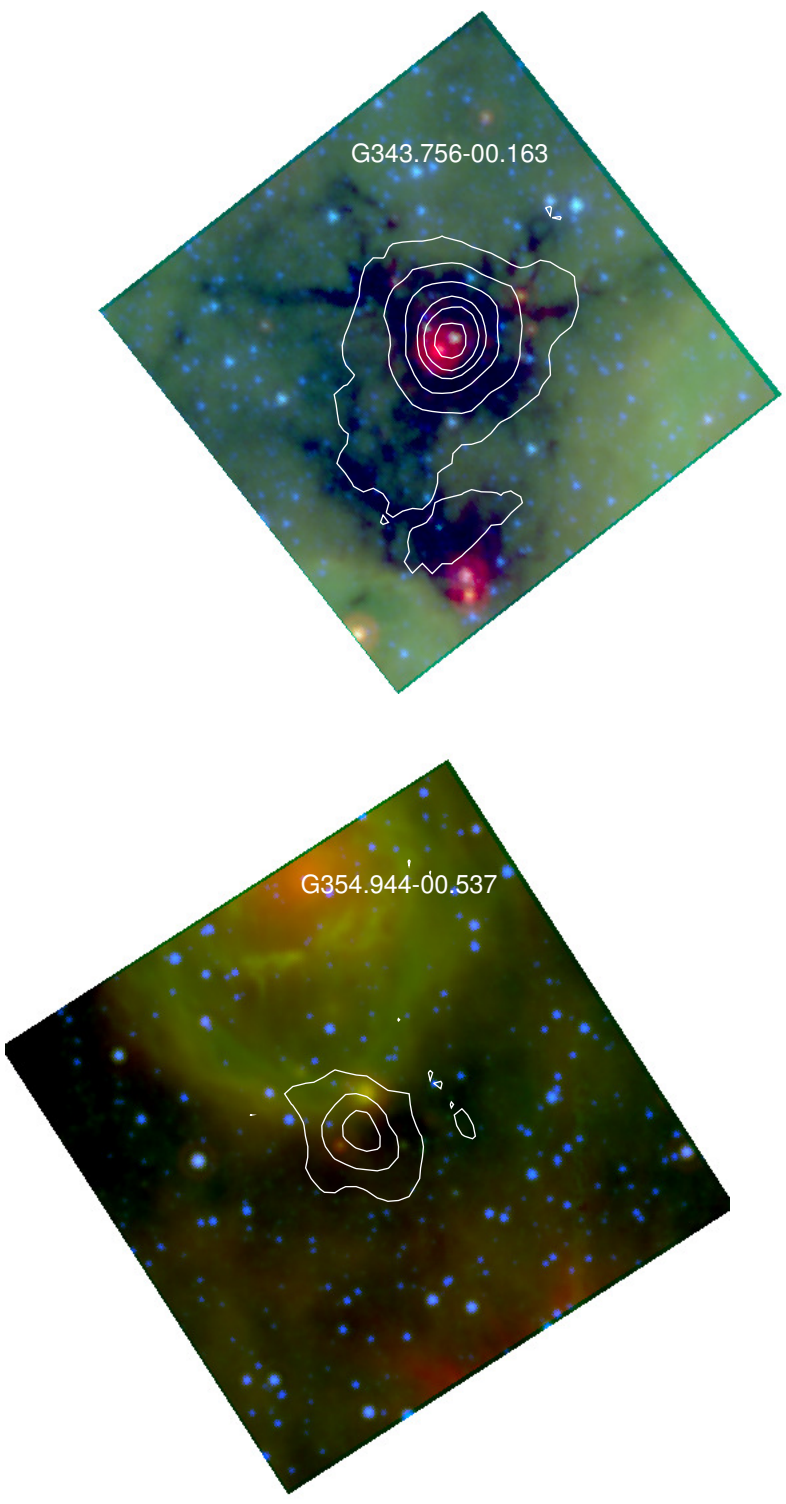

opacity can also cause line broadening (Beltrán et al. 2005). The line widths are measured from the averaged spectra in $38^{\prime \prime} \times 38^{\prime \prime}$ box (MOPRA beam size) around the clump's $\mathrm{N}_{2} \mathrm{H}^{+}$peak emission. To see any trends in the measured line widths of different spectral lines, we plot the number distributions of the line widths of the optically thin $\mathrm{H}^{13} \mathrm{CO}^{+}, \mathrm{HN}^{13} \mathrm{C}$ and $\mathrm{N}_{2} \mathrm{H}^{+}$lines in the left panel of Fig. 6. The line widths for optically thin $(\tau<1.0)$ $\mathrm{H}^{13} \mathrm{CO}^{+}$range between $1.4 \mathrm{~km} \mathrm{~s}^{-1}$ and $6.1 \mathrm{~km} \mathrm{~s}^{-1}$ with a median value of $3.2 \mathrm{~km} \mathrm{~s}^{-1}$. Similarly $\mathrm{HN}^{13} \mathrm{C}$ line widths range between $1.2 \mathrm{~km} \mathrm{~s}^{-1}$ and $4.5 \mathrm{~km} \mathrm{~s}^{-1}$ with a mean value of $2.8 \mathrm{~km} \mathrm{~s}^{-1}$. For half of the $\mathrm{N}_{2} \mathrm{H}^{+}$lines which are optically thin, the line widths range between $2.0 \mathrm{~km} \mathrm{~s}^{-1}$ and $4.8 \mathrm{~km} \mathrm{~s}^{-1}$, with a mean value of $3.3 \mathrm{~km} \mathrm{~s}^{-1}$, and for all $\mathrm{N}_{2} \mathrm{H}^{+}$lines including the optically thick ones the mean value is $3.2 \mathrm{~km} \mathrm{~s}^{-1}$. We conclude that there is a smooth distribution which peaks around $3 \mathrm{~km} \mathrm{~s}^{-1}$, while the line widths for all three molecules are smaller than $6 \mathrm{~km} \mathrm{~s}^{-1}$. In addition, the left panel of the Fig. 7 shows a tight correlation between the velocity widths of $\mathrm{N}_{2} \mathrm{H}^{+}$and $\mathrm{HN}^{13} \mathrm{C}$ lines which might be a sign that these lines come from the same region.

These $\mathrm{N}_{2} \mathrm{H}^{+}$values are also similar to those found for 159 clumps (quiescent to active) in Sanhueza et al. (2012), while 

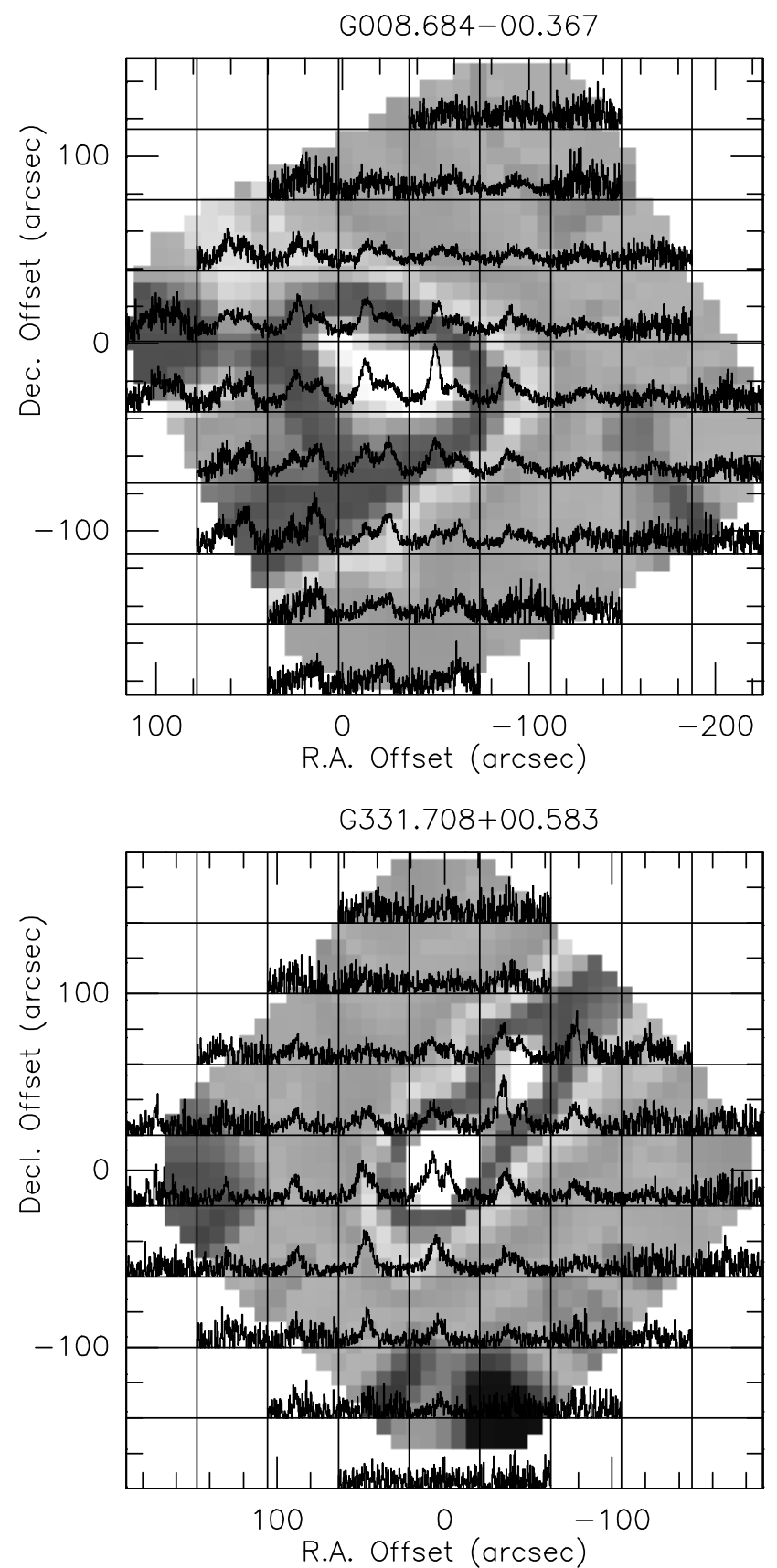

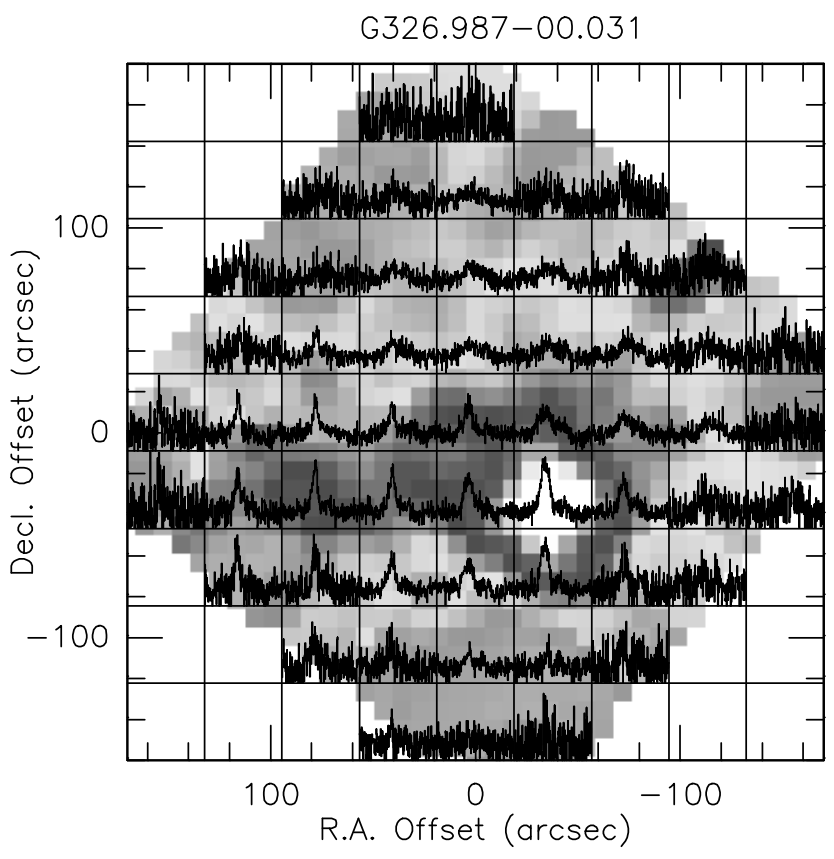

G353.066+00.452

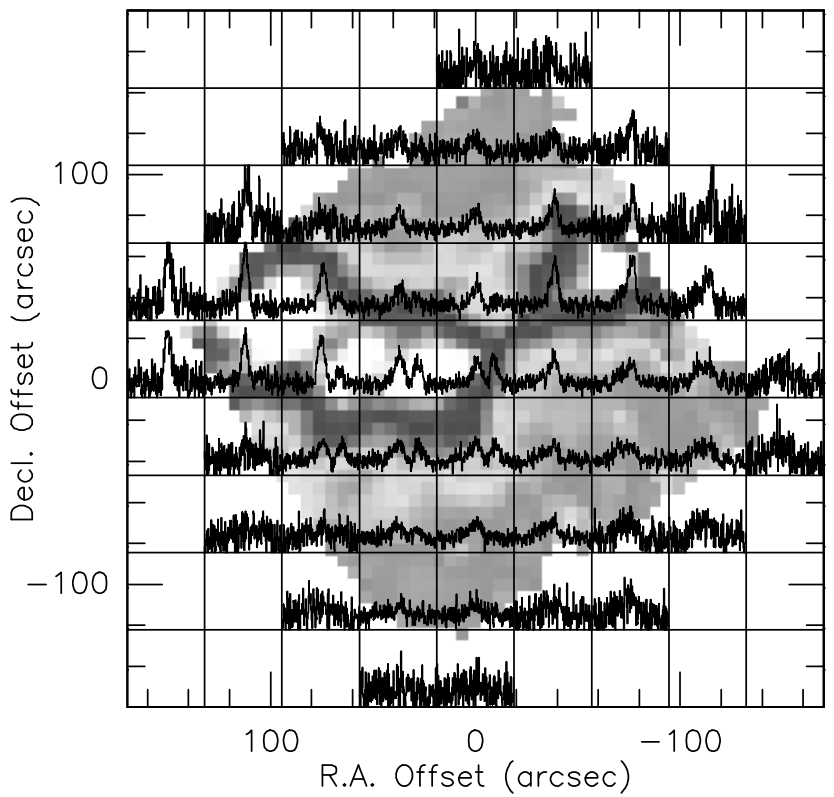

Fig. 4. $\mathrm{HCO}^{+}$map grid of four possible infall and outflow candidates (gridded to beam size) overlaid on the $\mathrm{HCO}^{+}$integrated intensity map.

the median $\mathrm{N}_{2} \mathrm{H}^{+}$line width is similar to the median values they found for more active $\left(3.0 \mathrm{~km} \mathrm{~s}^{-1}\right)$ and red clumps $\left(3.4 \mathrm{~km} \mathrm{~s}^{-1}\right)$ rather than quiescent $\left(2.8 \mathrm{~km} \mathrm{~s}^{-1}\right)$ or intermediate clumps $\left(2.7 \mathrm{~km} \mathrm{~s}^{-1}\right)$. Considering the small number of targets in our study, we can say that this difference is not significant. Furthermore, Sakai et al. (2008) studied 29 MSX objects, which are classified as mostly quiescent and intermediate or active clumps by Sanhueza et al. (2012), and found that the mean $\mathrm{N}_{2} \mathrm{H}^{+}$line width is about $2.4 \mathrm{~km} \mathrm{~s}^{-1}$ which is lower than the value we found. Similarly, Vasyunina et al. (2011) studied 37 clumps (varying from quiescent to active and red) and found a mean value of $1.8 \mathrm{~km} \mathrm{~s}^{-1}$ and Pirogov et al. (2003) found the mean $\mathrm{N}_{2} \mathrm{H}^{+}$line width value for 35 dense molecular cores to be $2.4 \mathrm{~km} \mathrm{~s}^{-1}$. On the other hand, for low-mass starless cores the average value is found to be $\sim 0.5 \mathrm{~km} \mathrm{~s}^{-1}$ (Benson et al. 1998).
Considering that our large beam size corresponds to $0.18 \mathrm{pc}$ at a distance of $1 \mathrm{kpc}$ and $1.85 \mathrm{pc}$ at a distance of $10 \mathrm{kpc}$, we might be covering a larger area for clumps at larger distances which might increase the line width as expected from Larson's laws (Larson 1981). To check if this was the case for our sample, we examined how the $\mathrm{N}_{2} \mathrm{H}^{+}$line widths are distributed by the distances. As can be seen in Fig. 8, large line widths cannot be explained by large distances. Therefore, the larger $\mathrm{N}_{2} \mathrm{H}^{+}$line widths might indicate initially turbulent clumps which favors the turbulence supported cores theory (McKee \& Tan 2003) for massive star formation. However, since we do not know if these clumps are starless or not, higher resolution data are needed to study the cores within these clumps.

Figure 6 shows the line width distributions for the optically thick $\mathrm{HCO}^{+}$and $\mathrm{HNC}$ lines. Line widths range between 
G014.632-00.577

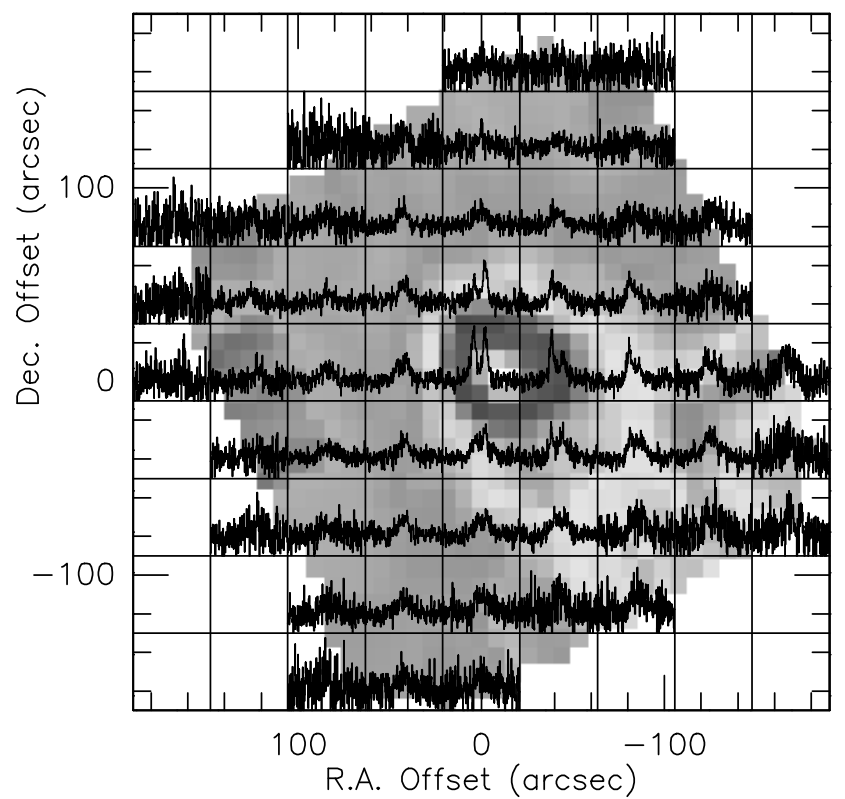

G317.867-00.151

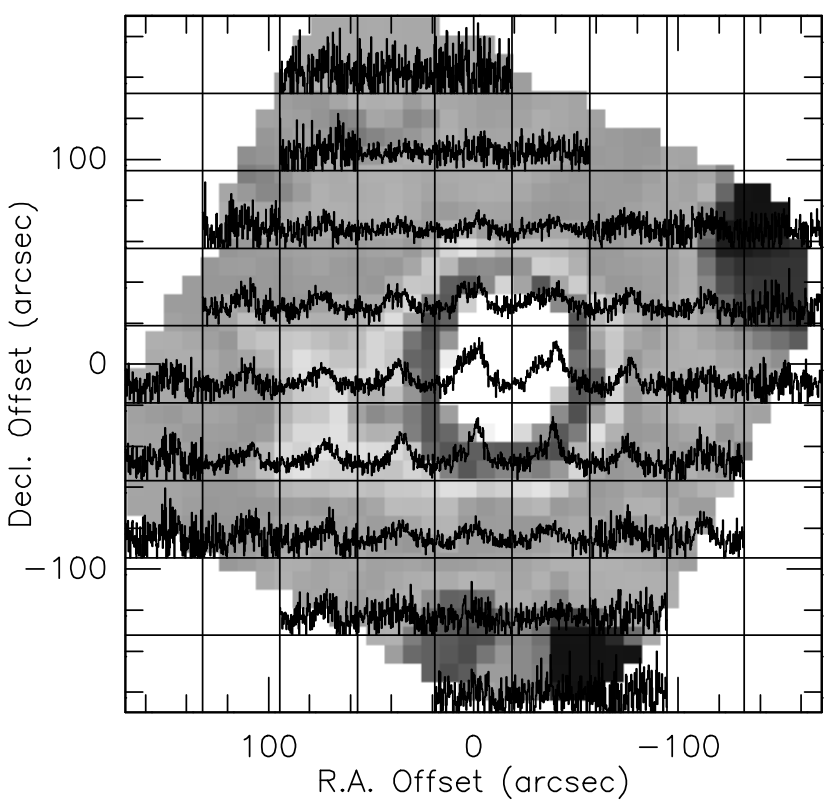

Fig. 5. $\mathrm{HCO}^{+}$map grid of two possible outflow candidates (gridded to beam size) overlaid on the $\mathrm{HCO}^{+}$integrated intensity map.

$1.2 \mathrm{~km} \mathrm{~s}^{-1}$ and $10.2 \mathrm{~km} \mathrm{~s}^{-1}$ and $1.4 \mathrm{~km} \mathrm{~s}^{-1}$ and $7.6 \mathrm{~km} \mathrm{~s}^{-1}$ and the mean values are $4.1 \mathrm{~km} \mathrm{~s}^{-1}$ and $4.0 \mathrm{~km} \mathrm{~s}^{-1}$ for the $\mathrm{HCO}^{+}$and $\mathrm{HNC}$ lines, respectively. The larger line widths compared to the $\mathrm{N}_{2} \mathrm{H}^{+}$lines can be explained with larger optical depths. Also as can be clearly seen in some sources (e.g., G014.194-00.194, G331.708+00.583) line wings can cause larger line widths possibly originating from outflows. G014.194-00.194, G331.708+00.583_1, and G343.756-00.164 with $\mathrm{SiO}$ signal within the range of $9 \bar{\sigma}, \mathrm{G} 329.029-00.206$ with $12 \sigma$, and $\mathrm{G} 351.444+00.659$ with $15 \sigma$ are the most promising regions to look for outflows.

In general, the $\mathrm{SiO}$ lines, whose widths are also shown in Fig. 6 are broader than the $\mathrm{H}^{13} \mathrm{CO}^{+}$and $\mathrm{HN}^{13} \mathrm{C}$ lines and range between $4.4 \mathrm{~km} \mathrm{~s}^{-1}$ and $14.0 \mathrm{~km} \mathrm{~s}^{-1}$ with a mean value of $7.2 \mathrm{~km} \mathrm{~s}^{-1}$, as expected when there are shocks or outflows (see the right panel of Fig. 7). The majority of the sources, however, show no strong outflow signatures in other molecular lines which indicates that the $\mathrm{SiO}$ emission might not be due to the shocks in outflows but instead are due to colliding flows. SiO has been studied toward the mini-starburst region W43 where extended $\mathrm{SiO}$ emission is observed with line widths $>6 \mathrm{~km} \mathrm{~s}^{-1}$. The emission is not connected to any specific outflow activities and is proposed to originate from large-scale interactions during the cloud formation process (Nguyen-Luong et al. 2013; Louvet et al. 2016). Therefore, extended SiO emission might not be affected by star formation activity in all cases and it might represent remnant emission from the cloud formation process. In our study, only eleven sources have $\mathrm{SiO}$ detections over $3 \sigma$ and only five of them are over $6 \sigma$. Although the line widths are large enough to give clues about outflows, it is difficult to derive conclusions about the nature of the $\mathrm{SiO}$ emission in these clumps without higher sensitivity observations, considering there is no detection of velocity gradients detected for the $\mathrm{SiO}$ line.

To check the velocity gradients, we constructed velocity maps of the sources from the $\mathrm{HCO}^{+}$and $\mathrm{N}_{2} \mathrm{H}^{+}$lines as shown in Fig. 9. There are 25 sources which show only small velocity gradients of up to $\sim 2 \mathrm{~km} \mathrm{~s}^{-1}$ across each clump (a few parsecs) which are consistent with the velocity gradient values observed in other clouds (Belloche 2013). High-resolution interferometric observations at core scales could reveal $\sim 5$ times more velocity gradients (Belloche 2013) in these regions and reveal the outflows originating from individual cores.

Due to the different gas-grain chemistry of the molecular species, we can use different lines to trace specific motions such as infalls outflow, rotation or turbulent motions. Studies show that outflows from intermediate- and high-mass protostars can generate parsec-scale velocity gradients (e.g., Benedettini et al. 2004), and rotation of the cloud and cores and turbulence can also affect the observed velocity gradients (e.g., Belloche 2013). For instance, $\mathrm{HCO}^{+}$enhancement is known to occur in ionized gas due to strong shocks (Dickinson et al. 1980; White et al. 1987; Rawlings et al. 2004; Girart et al. 2005) and high-velocity $\mathrm{HCO}^{+}$emission, line wings, and velocity gradients are already observed in many star-forming regions with outflows (e.g., Sandqvist et al. 1982; Garden \& Carlstrom 1992; Jørgensen et al. 2004). The variation of the $\mathrm{N}_{2} \mathrm{H}^{+}$line velocities is mostly used to trace the accretion flows or rotation of the dense gas (e.g., Di Francesco et al. 2001; Kirk et al. 2013; Dhabal et al. 2018). For this study, with the limited resolution of single-dish observations, however, we note that the observed velocity gradients are generated by the bulk motion of the common envelope, rather than individual cores within the clump.

\subsection{Integrated intensities}

The relationships between the intensities of the different molecules can be used to understand the chemical evolution in star-forming regions. We find the mean integrated intensities to be $5.12 \mathrm{~K} \mathrm{~km} \mathrm{~s}^{-1}, 9.79 \mathrm{~K} \mathrm{~km} \mathrm{~s}^{-1}, 4.22 \mathrm{~K} \mathrm{~km} \mathrm{~s}^{-1}$, and $6.92 \mathrm{~K} \mathrm{~km} \mathrm{~s}^{-1}$ for the $\mathrm{HCO}^{+}, \mathrm{N}_{2} \mathrm{H}^{+}, \mathrm{HNC}$, and $\mathrm{HCN}$ lines, respectively, as shown in Table 5. Since $\mathrm{N}_{2} \mathrm{H}^{+}$is a convenient molecule to study the kinematics of cold, dense starforming regions (Caselli et al. 2002) due to the resistance of depletion under these conditions, and also traces the gas with a density one order of magnitude higher than what $\mathrm{HCO}^{+}$does (Shirley 2015), we compared the mean integrated intensity of 

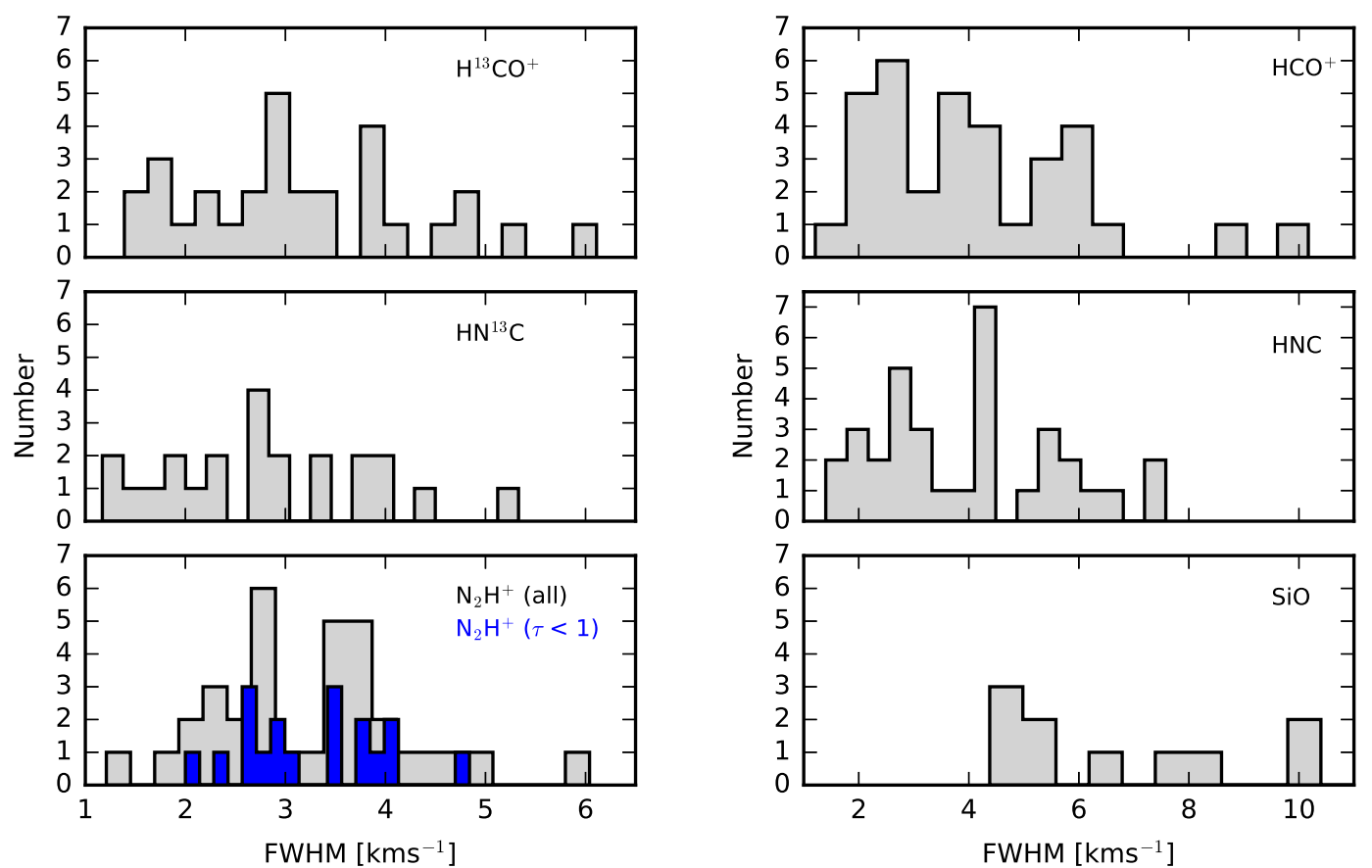

Fig. 6. Histograms of the number distributions of line widths. Left column: for the $\mathrm{H}^{13} \mathrm{CO}^{+}, \mathrm{HN}^{13} \mathrm{C}$, and $\mathrm{N}_{2} \mathrm{H}^{+}$lines. Optically thin $\mathrm{N}_{2} \mathrm{H}^{+}$lines are plotted in blue. Right column: for the $\mathrm{HCO}^{+}, \mathrm{HNC}$, and $\mathrm{SiO}$ lines.
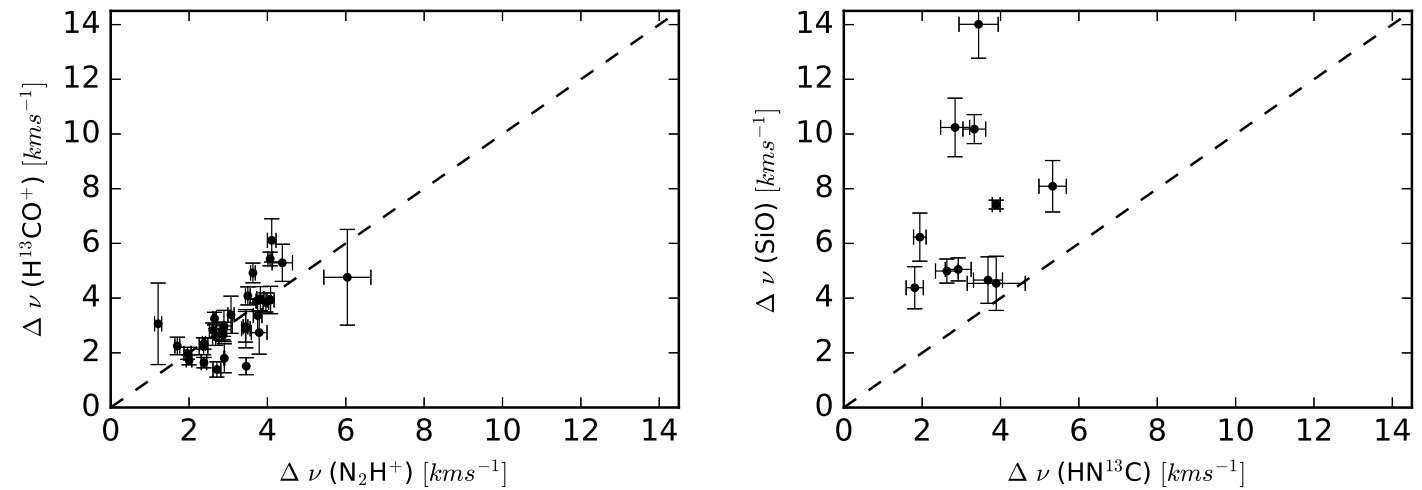

Fig. 7. Velocity widths of several molecular lines are compared. A ratio of one is shown as a black dashed line.

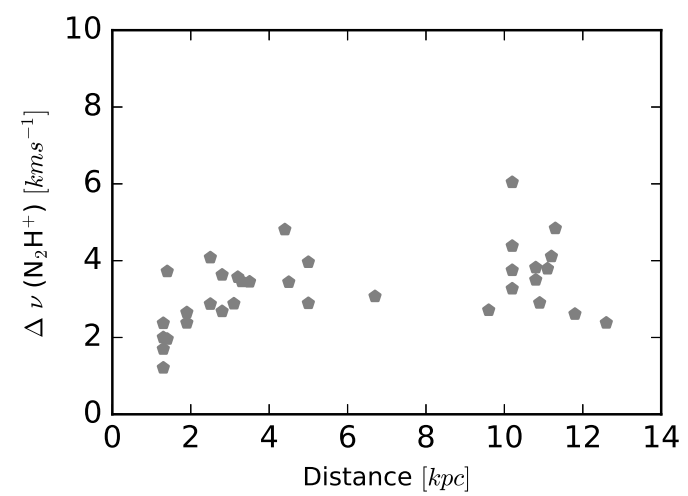

Fig. 8. $\mathrm{N}_{2} \mathrm{H}^{+}$line width distribution of the clumps according to their kinematic distances.

this line to other star-forming regions in order to derive information about the evolutionary stages of these clumps. With the mean integrated intensity of $9.79 \pm 0.28 \mathrm{~K} \mathrm{~km} \mathrm{~s}^{-1}$ (median is $6.62 \pm 1.63 \mathrm{~K} \mathrm{~km} \mathrm{~s}^{-1}$ ), the selected sample is at similar evolutionary levels with the quiescent and intermediate clumps studied in Vasyunina et al. (2011). They studied 15 IRDCs which were classified as quiescent, mid, and active with mean integrated intensities of $4.7 \mathrm{~K} \mathrm{~km} \mathrm{~s}^{-1}, 7.1 \mathrm{~K} \mathrm{~km} \mathrm{~s}^{-1}$, and $10.6 \mathrm{~K} \mathrm{~km} \mathrm{~s}^{-1}$, respectively.

In order to see how the intensities of the different molecules are correlated we plotted the intensities of $\mathrm{HCO}^{+}-\mathrm{HNC}$, HNC$\mathrm{HCN}, \mathrm{HCO}^{+}-\mathrm{HCN}$, and $\mathrm{N}_{2} \mathrm{H}^{+}-\mathrm{HCN}$ in Fig. 10. The linear fit results and $r$-values (shown on the panels) indicate that there is a tight correlation between emission from these molecules during the evolution of the clumps. We note, however, that the tight correlation is also caused by the data points corresponding to G351.444+00.659 which has large intensity values. Excluding this data point gives still a tight correlation $(r=91)$ between $\mathrm{HNC}$ and $\mathrm{HCO}^{+}$, while the correlation between other molecules is decreases $(r \sim 0.70)$. Similar correlations between emission from these molecules had been found for other IRDCs (e.g., Liu et al. 2013a).

The median intensity ratios are $1.30 \pm 0.17,1.40 \pm 0.56$, and $0.61 \pm 0.11$ for $I_{\mathrm{HCN}} / I_{\mathrm{HCO}^{+}}, I_{\mathrm{N}_{2} \mathrm{H}^{+}} / I_{\mathrm{HCN}}$, and $I_{\mathrm{HNC}} / I_{\mathrm{HCN}}$, 

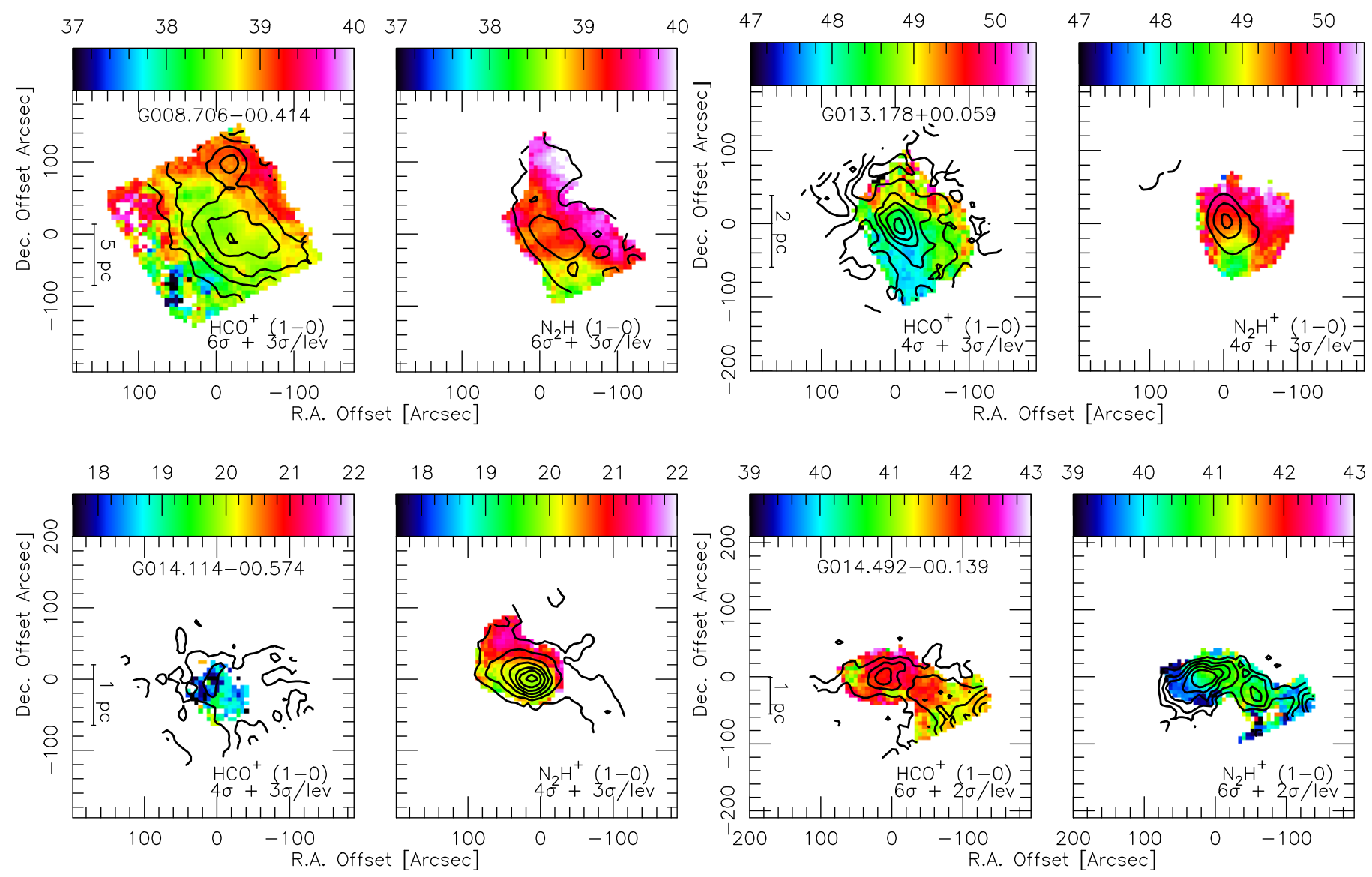

Fig. 9. Velocity (moment 1) maps overlaid with the intensity contours of the same line showing the velocity gradients in each clump. The top color bar is in units of $\mathrm{km} \mathrm{s}^{-1}$. Contour levels are shown on each image. All the velocity maps are generated with $3 \sigma$ level of the average rms of $\mathrm{HCO}^{+}$and $\mathrm{N}_{2} \mathrm{H}^{+}$channel maps. We show only a few examples here; maps for all the sources can be found in Fig. A.1.

Table 5. Statistical properties of the intensities.

\begin{tabular}{lllll}
\hline \hline & $I_{\mathrm{HCO}^{+}}$ & $I_{\mathrm{N}_{2} \mathrm{H}^{+}}$ & $I_{\mathrm{HNC}}$ & $I_{\mathrm{HCN}}$ \\
\hline Mean & $5.12(0.03)$ & $9.79(0.28)$ & $4.22(0.04)$ & $6.92(0.37)$ \\
Median & $3.67(0.09)$ & $6.62(1.63)$ & $3.10(0.40)$ & $5.87(1.67)$ \\
Std. & 5.30 & 10.52 & 4.45 & 8.13 \\
\hline
\end{tabular}

respectively. The value found for the ratio of $I_{\mathrm{HCN}} / I_{\mathrm{HCO}^{+}}$is similar to the value of $1.67 \pm 0.83$ found in the starburst ring of NGC 1097 (Hsieh et al. 2012), while it is bigger than the mean value of 0.67 found for 14 IRDCs in Liu et al. (2013a). This behavior indicates that the $I_{\mathrm{HCN}} / I_{\mathrm{HCO}^{+}}$ratio is not dependent on evolution but rather other mechanisms such as $\mathrm{X}$ ray ionization by HII regions. Massive stars in the vicinity of the IRDCs and clumps may also play a role by affecting the intensities as also stated in Hsieh et al. (2012) and Liu et al. (2013a). On the other hand, the ratio of $I_{\mathrm{N}_{2} \mathrm{H}^{+}} / I_{\mathrm{HCN}}$ was found to be $1.40 \pm 0.56$, which is similar to the value of $\sim 1.5$ found in Liu et al. (2013a), which might indicate that the IRDCs examined in both studies are at similar evolutionary stages.

\subsection{Column density ratios}

Table B.5 shows the column density ratios of different molecules. The mean column density ratios of $\mathrm{N}(\mathrm{HNC}) / \mathrm{N}\left(\mathrm{HCO}^{+}\right)$, $\mathrm{N}\left(\mathrm{N}_{2} \mathrm{H}^{+}\right) / \mathrm{N}\left(\mathrm{HCO}^{+}\right), \mathrm{N}\left(\mathrm{N}_{2} \mathrm{H}^{+}\right) / \mathrm{N}(\mathrm{HNC}), \mathrm{N}(\mathrm{HNC}) / \mathrm{N}(\mathrm{HCN})$, and
$\mathrm{N}(\mathrm{HCN}) / \mathrm{N}\left(\mathrm{HCO}^{+}\right)$are $2.3 \pm 0.1,0.8 \pm 0.1,1.3 \pm 0.7,8.9 \pm 1.0$ and $0.8 \pm 0.23$, respectively (median and standard deviation values are also shown in Table B.5).

The median ratio of $\mathrm{N}(\mathrm{HNC}) / \mathrm{N}\left(\mathrm{HCO}^{+}\right), 1.2 \pm 0.2$, is similar to the value, $1.7 \pm 0.2$ found by Liu et al. (2013a) for 14 IRDCs. On the other hand, for massive complex star-forming regions in the Galactic Plane (e.g., W49, W51, G10.62-0.39, and $\mathrm{G} 34.3+0.1$ ) this ratio was found to be only $0.5 \pm 0.3$ by Godard et al. (2010) which indicates that the ratio of $\mathrm{N}(\mathrm{HNC}) / \mathrm{N}\left(\mathrm{HCO}^{+}\right)$might be affected by the ambient environment. Hence, it is difficult to use it as an indicator for early evolution.

On the other hand, the column density ratio of $\mathrm{N}(\mathrm{HCN}) / \mathrm{N}\left(\mathrm{HCO}^{+}\right)$with a median of 0.2 seems similar for most of the sources except G014.492-00.039, G338.786+00.476, and G353.417-00.079_2 which have quite high ratios $(3.2,4.8$, 9.4 , respectively) compared to others. This behavior is a result of lower limit column density estimations for $\mathrm{HCO}^{+}$since we were not be able to calculate optical depths for these sources. Similarly, due to the emission line anomalies of HCN, and not being able to estimate an optical depth for half of the sources, we might also be underestimating the HCN column densities. This effect will result in a systematic error of an order of magnitude in the determined column densities. With these constraints, we can say that for all the sources except the three mentioned above, $\mathrm{N}(\mathrm{HCN}) / \mathrm{N}\left(\mathrm{HCO}^{+}\right)$is almost same within the errors in all clumps. Considering that some of the clumps are associated with protostellar sources, and YSOs while some others with massive star forming (MSF) regions, we can say that 

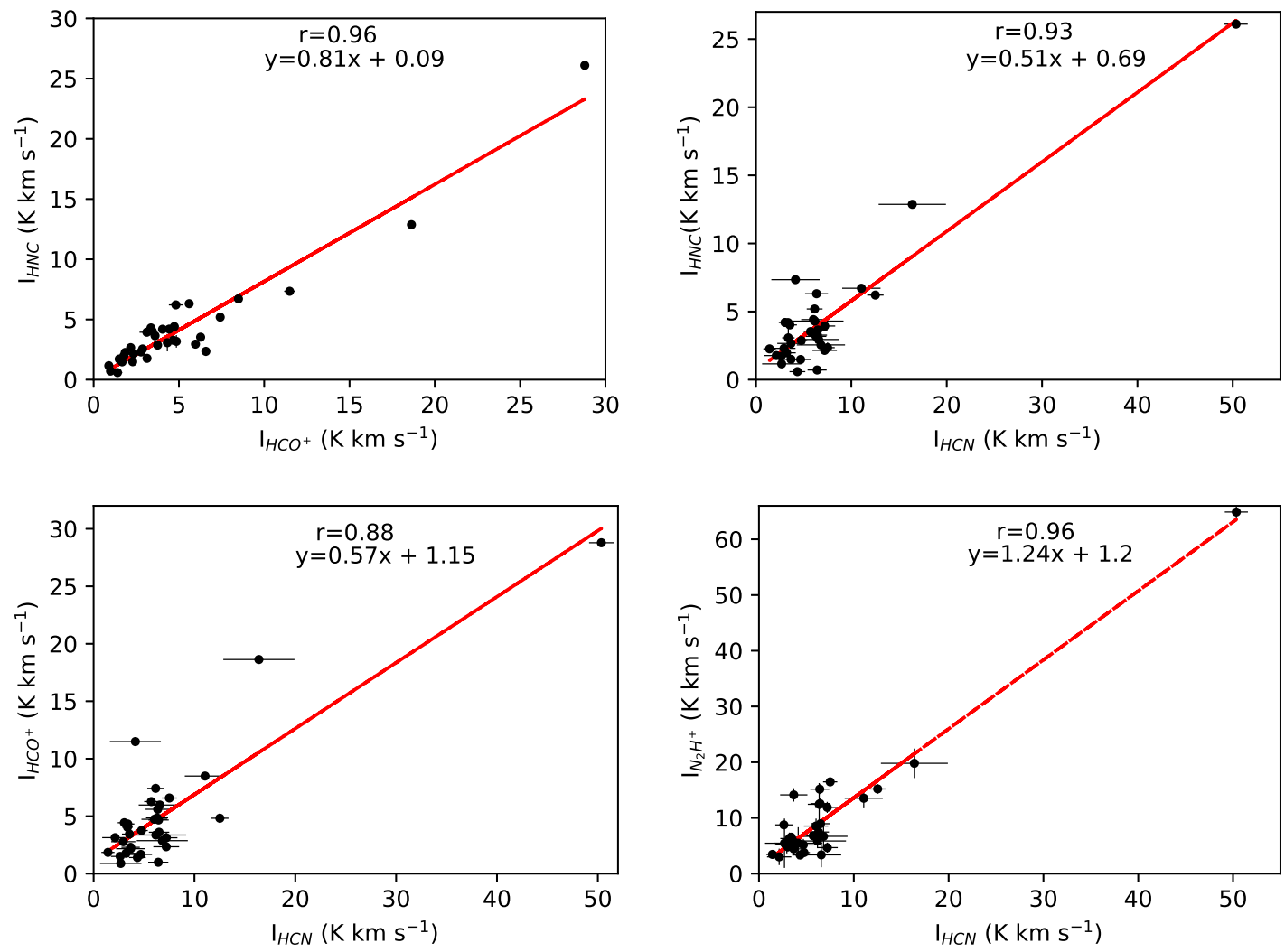

Fig. 10. Integrated intensity relations between $\mathrm{HCO}^{+}, \mathrm{HNC}$, and $\mathrm{HCN}$. The red line represent the linear fit.

this column density ratio is not strongly affected by the different evolutionary phases.

Similarly, we do not see any significant difference in the column density ratios of other molecules. These results, however, are based on small number of sources and considering the possible systematic errors especially for HCN column densities, and in some cases for $\mathrm{HCO}^{+}$column densities as well, the column density ratios cannot be used to claim clear evolutionary phase differences between the studied clumps.

\section{Summary}

The physical and chemical properties of 30 infrared-dark highmass clumps at $8 / 24 \mu \mathrm{m}$ from the ATLASGAL survey were investigated using MALT90 Survey data. The molecular lines $\mathrm{HCO}^{+}(1-0), \mathrm{HNC}(1-0)$, and $\mathrm{N}_{2} \mathrm{H}^{+}(1-0)$ were used as density tracers, along with their isotopologues $\mathrm{H}^{13} \mathrm{CO}^{+}(1-0)$ and $\mathrm{HN}^{13} \mathrm{C}(1-0)$ and, $\mathrm{SiO}(2-1)$ was used as an outflow tracer. Velocity maps and spectral line profiles for 30 sources were investigated. Column densities and the ratios of column densities were calculated in order to determine the nature of these sources which are at early stages of potential high-mass star formation.

1. We find 13 sources with blue profiles in both/either $\mathrm{HCO}^{+}$and/or $\mathrm{HNC}$ lines which might indicate infall and estimated clump mass infall rates ranging between $0.2 \times 10^{-3} M_{\odot} \mathrm{yr}^{-1}-1.8 \times 10^{-2} M_{\odot} \mathrm{yr}^{-1}$.

2. The integrated intensities of the $\mathrm{HCO}^{+}, \mathrm{N}_{2} \mathrm{H}^{+}, \mathrm{HNC}$, and $\mathrm{HCN}$ lines have tight correlations with each other which indicate that they might share a similar chemical evolution during the early stages of IRDC formation. Large line widths for the $\mathrm{N}_{2} \mathrm{H}^{+}$line might indicate turbulence, and large widths of the $\mathrm{HCO}^{+}, \mathrm{HNC}$, and $\mathrm{SiO}$ lines might indicate outflow activities.
3. $\mathrm{HCO}^{+}$and $\mathrm{HCN}$ line emission is found to be optically thick, with median values of 18.08, and 15.50, respectively, while line emission from their isotopologues $\mathrm{H}^{13} \mathrm{CO}^{+}$and $\mathrm{HN}^{13} \mathrm{C}$ is optically thin with median values of 0.35 and 0.30 , respectively. Sixteen sources have optically thin $\mathrm{N}_{2} \mathrm{H}^{+}$lines, while the median value for all sources is $1.00 \pm 0.18$.

4. The excitation temperature, $T_{\mathrm{ex}}$ is calculated as $<24 \mathrm{~K}$ for all sources as expected in the cold dense regions within IRDCs.

5. Eleven sources have a significant $\mathrm{SiO}$ detection (above $3 \sigma$ ) but the $\mathrm{S} / \mathrm{N}$ is not sufficient enough to do further analysis.

6. We used the $\mathrm{HCO}^{+}$and $\mathrm{N}_{2} \mathrm{H}^{+}$lines to investigate the kinematics within the clumps, and only a few sources (G008.706-00.414, G013.178+00.059, G014.114-00.574) show small velocity gradients over the clumps with a maximum of $2 \mathrm{~km} \mathrm{~s}^{-1}$ in both lines.

7. Some sources might have multiple cores, outflow, and infall activities based on the line profiles and kinematics. In order to understand the true nature of these clumps, however, it is necessary to obtain high angular resolution and high sensitivity data and obtain radiative transfer models to reproduce the line profiles.

Acknowledgements. This work is supported by the STARFORM Sinergia Project CH:CRSII2_160759 funded by the Swiss National Science Foundation. The authors also thank Dr. Joseph Hora for careful reading of the manuscript and valuable comments that helped improve the paper. This work has made use of the data products from the Millimetre Astronomy Legacy Team $90 \mathrm{GHz}$ (MALT90) survey and the APEX Telescope Large Area Survey of the Galaxy (ATLASGAL), which is a collaboration between the Max-Planck-Gesellschaft, the European Southern Observatory (ESO) and the Universidad de Chile; and also used observations made with the Spitzer Space Telescope, which is operated by the Jet Propulsion Laboratory, California Institute of Technology, under contract with NASA. This research has made use of NASA's Astrophysics Data System Abstract Service; and the SIMBAD data base, operated at CDS, Strasbourg, France. We thank the anonymous referee for providing valuable comments and suggestions. 


\section{References}

Belloche, A. 2013, in EAS Pub. Ser., eds. P. Hennebelle, \& C. Charbonnel, 62, 25

Beltrán, M. T., Cesaroni, R., Neri, R., et al. 2005, A\&A, 435, 901

Beltrán, M. T., Cesaroni, R., Codella, C., et al. 2006, Nature, 443, 427

Benedettini, M., Molinari, S., Testi, L., \& Noriega-Crespo, A. 2004, MNRAS, 347,295

Benson, P. J., Caselli, P., \& Myers, P. C. 1998, ApJ, 506, 743

Beuther, H., Sridharan, T. K., \& Saito, M. 2005, ApJ, 634, L185

Beuther, H., Churchwell, E. B., McKee, C. F., \& Tan, J. C. 2007, Protostars and Planets V, 165,

Beuther, H., Henning, T., Linz, H., et al. 2015, A\&A, 581, A119

Bica, E., Dutra, C. M., Soares, J., \& Barbuy, B. 2003, A\&A, 404, 223

Busquet, G., Estalella, R., Palau, A., et al. 2016, ApJ, 819, 139

Caselli, P., Walmsley, C. M., Zucconi, A., et al. 2002, ApJ, 565, 344

Cesaroni, R., Felli, M., Testi, L., Walmsley, C. M., \& Olmi, L. 1997, A\&A, 325, 725

Chambers, E. T., Jackson, J. M., Rathborne, J. M., \& Simon, R. 2009, ApJS, 181, 360

Chen, X., Shen, Z. Q., Li, J. J., Xu, Y., \& He, J. H. 2010, ApJ, 710, 150

Chira, R.-A., Smith, R. J., Klessen, R. S., Stutz, A. M., \& Shetty, R. 2014, MNRAS, 444, 874

Contreras, Y., Schuller, F., Urquhart, J. S., et al. 2013, A\&A, 549, A45

Contreras, Y., Sanhueza, P., Jackson, J. M., et al. 2018, ApJ, 861, 14

Csengeri, T., Bontemps, S., Schneider, N., Motte, F., \& Dib, S. 2011, A\&A, 527 A135

Csengeri, T., Urquhart, J. S., Schuller, F., et al. 2014, A\&A, 565, A75

Csengeri, T., Leurini, S., Wyrowski, F., et al. 2016, A\&A, 586, A149

Cyganowski, C. J., Koda, J., Rosolowsky, E., et al. 2013, ApJ, 764, 61

De Vries, C. H., \& Myers, P. C. 2005, ApJ, 620, 800

Dhabal, A., Mundy, L. G., Rizzo, M. J., Storm, S., \& Teuben, P. 2018, ApJ, 853 169

Dickinson, D. F., Rodriguez Kuiper, E. N., Dinger, A. S. C., \& Kuiper, T. B. H. 1980, ApJ, 237, L43

di Francesco, J., Evans, II, N. J., Caselli, P., et al. 2007, Protostars and Planets V, 17

Di Francesco, J., Myers, P. C., Wilner, D. J., Ohashi, N., \& Mardones, D. 2001, ApJ, 562, 770

Evans, II, N. J. 1999, ARA\&A, 37, 311

Fontani, F., Palau, A., Caselli, P., et al. 2011, A\&A, 529, L7

Foster, J. B., Jackson, J. M., Barnes, P. J., et al. 2011, ApJS, 197, 25

Foster, J. B., Rathborne, J. M., Sanhueza, P., et al. 2013, PASA, 30, e038

Garden, R. P., \& Carlstrom, J. E. 1992, ApJ, 392, 602

Giannetti, A., Wyrowski, F., Brand, J., et al. 2014, A\&A, 570, A65

Ginsburg, A., \& Mirocha, J. 2011, Astrophysics Source Code Library [record ascl:1109.001]

Girart, J. M., Viti, S., Estalella, R., \& Williams, D. A. 2005, A\&A, 439, 601

Godard, B., Falgarone, E., Gerin, M., Hily-Blant, P., \& de Luca, M. 2010, A\&A 520, A20

Greve, T. R., Papadopoulos, P. P., Gao, Y., \& Radford, S. J. E. 2009, ApJ, 692, 1432

He, Y.-X., Zhou, J.-J., Esimbek, J., et al. 2015, MNRAS, 450, 1926

Heyer, M., Gutermuth, R., Urquhart, J. S., et al. 2016, A\&A, 588, A29

Hoare, M. G., Purcell, C. R., Churchwell, E. B., et al. 2012, PASP, 124, 939

Hoq, S., Jackson, J. M., Foster, J. B., et al. 2013, ApJ, 777, 157

Hsieh, P.-Y., Ho, P. T. P., Kohno, K., Hwang, C.-Y., \& Matsushita, S. 2012, ApJ, 747, 90

Jackson, J. M., Rathborne, J. M., Foster, J. B., et al. 2013, PASA, 30, e057

Jørgensen, J. K., Hogerheijde, M. R., Blake, G. A., et al. 2004, A\&A, 415, 1021

Kirk, H., Myers, P. C., Bourke, T. L., et al. 2013, ApJ, 766, 115

König, C., Urquhart, J. S., Csengeri, T., et al. 2017, A\&A, 599, A139
Lada, C. J., \& Lada, E. A. 2003, ARA\&A, 41, 57

Larson, R. B. 1981, MNRAS, 194, 809

Li, Z. Y., Banerjee, R., Pudritz, R. E., et al. 2014, Protostars and Planets VI, 173

Liu, X.-L., Wang, J.-J., \& Xu, J.-L. 2013a, MNRAS, 431, 27

Liu, T., Wu, Y., Wu, J., Qin, S.-L., \& Zhang, H. 2013b, MNRAS, 436, 1335

Liu, T., Lacy, J., Li, P. S., et al. 2017, ApJ, 849, 25

Longmore, S. N., Pillai, T., Keto, E., Zhang, Q., \& Qiu, K. 2011, ApJ, 726, 97

Loughnane, R. M., Redman, M. P., Thompson, M. A., et al. 2012, MNRAS, 420, 1367

Louvet, F., Motte, F., Gusdorf, A., et al. 2016, A\&A, 595, A122

Mardones, D., Myers, P. C., Tafalla, M., et al. 1997, ApJ, 489, 719

McKee, C. F., \& Tan, J. C. 2003, ApJ, 585, 850

Miettinen, O. 2014, A\&A, 562, A3

Motte, F., Andre, P., \& Neri, R. 1998, A\&A, 336, 150

Motte, F., Bontemps, S., Schilke, P., et al. 2007, A\&A, 476, 1243

Motte, F., Bontemps, S., \& Louvet, F. 2018, ARA\&A, 56, 41

Müller, H. S. P., Thorwirth, S., Roth, D. A., \& Winnewisser, G. 2001, A\&A, 370, L49

Müller, H. S. P., Schlöder, F., Stutzki, J., \& Winnewisser, G. 2005, J. Mol. Struct., 742,215

Myers, P. C., Mardones, D., Tafalla, M., Williams, J. P., \& Wilner, D. J. 1996, ApJ, 465, L133

Narayanan, G., Moriarty-Schieven, G., Walker, C. K., \& Butner, H. M. 2002 , ApJ, 565, 319

Nguyen-Luong, Q., Motte, F., Carlhoff, P., et al. 2013, ApJ, 775, 88

Peretto, N., Fuller, G. A., Duarte-Cabral, A., et al. 2013, A\&A, 555, A112

Pirogov, L., Zinchenko, I., Caselli, P., Johansson, L. E. B., \& Myers, P. C. 2003, A\&A, 405, 639

Povich, M. S., \& Whitney, B. A. 2010, ApJ, 714, L285

Povich, M. S., Townsley, L. K., Robitaille, T. P., et al. 2016, ApJ, 825, 125

Rathborne, J. M., Whitaker, J. S., Jackson, J. M., et al. 2016, PASA, 33, e030

Rawlings, J. M. C., Redman, M. P., Keto, E., \& Williams, D. A. 2004, MNRAS, 351,1054

Rygl, K. L. J., Wyrowski, F., Schuller, F., \& Menten, K. M. 2013, A\&A, 549, A5

Sakai, T., Sakai, N., Kamegai, K., et al. 2008, ApJ, 678, 1049

Sandqvist, A., Wootten, A., Loren, R. B., Friberg, P., \& Hjalmarson, A. 1982, in Regions of Recent Star Formation, eds. R. S. Roger, \& P. E. Dewdney, Astrophys. Space Sci. Lib., 93, 307

Sanhueza, P., Jackson, J. M., Foster, J. B., et al. 2012, ApJ, 756, 60

Savage, C., Apponi, A. J., Ziurys, L. M., \& Wyckoff, S. 2002, ApJ, 578, 211

Schilke, P., Walmsley, C.M., Pineau des Forets, G., \& Flower, D.R. 1997, A\&A, 321, 293

Schöier, F. L., van der Tak, F. F. S., van Dishoeck, E. F., \& Black, J. H. 2005, A\&A, 432, 369

Schuller, F., Menten, K. M., Contreras, Y., et al. 2009, A\&A, 504, 415

Shirley, Y. L. 2015, PASP, 127, 299

Smith, R. J., Shetty, R., Beuther, H., Klessen, R. S., \& Bonnell, I. A. 2013, ApJ, 771,24

Sun, Y., \& Gao, Y. 2009, MNRAS, 392, 170

Sánchez-Monge, 2011, PhD Thesis, Universitat de Barcelona, Spain

Urquhart, J. S., Busfield, A. L., Hoare, M. G., et al. 2008, A\&A, 487, 253

Urquhart, J. S., König, C., Giannetti, A., et al. 2018, MNRAS, 473, 1059

Vasyunina, T., Linz, H., Henning, T., et al. 2011, A\&A, 527, A88

White, G. J., Rainey, R., Hayashi, S. S., \& Kaifu, N. 1987, A\&A, 173, 337

Wienen, M., Wyrowski, F., Menten, K. M., et al. 2015, A\&A, 579, A91

Wood, D. O. S., \& Churchwell, E. 1989, ApJS, 69, 831

Wu, Y., Henkel, C., Xue, R., Guan, X., \& Miller, M. 2007, ApJ, 669, L37

Wu, Y. W., Sato, M., Reid, M. J., et al. 2014, A\&A, 566, A17

Yu, N., \& Wang, J.-J. 2015, MNRAS, 451, 2507

Zhou, S. 1992, ApJ, 394, 204

Zinnecker, H., \& Yorke, H. W. 2007, ARA\&A, 45, 481 


\section{Appendix A: Integrated intensity maps and line spectra}
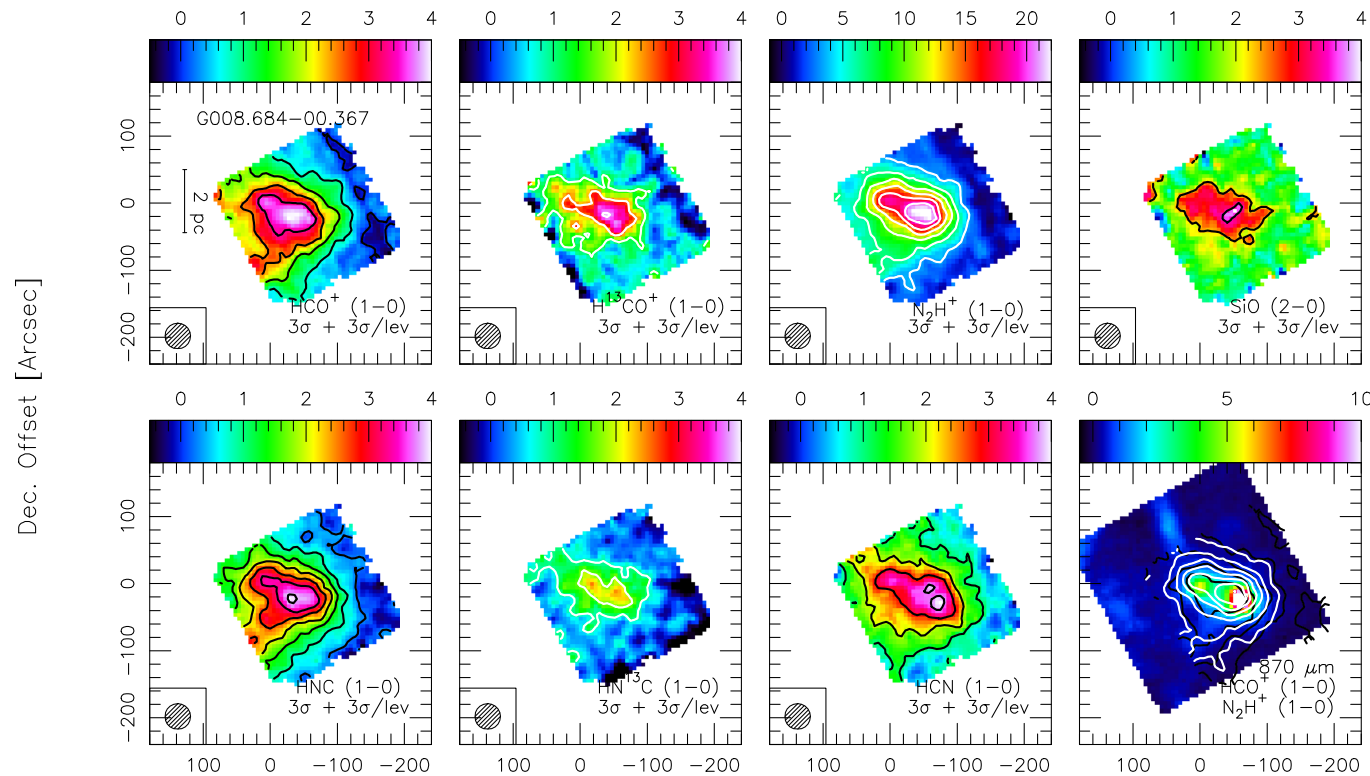

R.A. Offset [Arcsec]

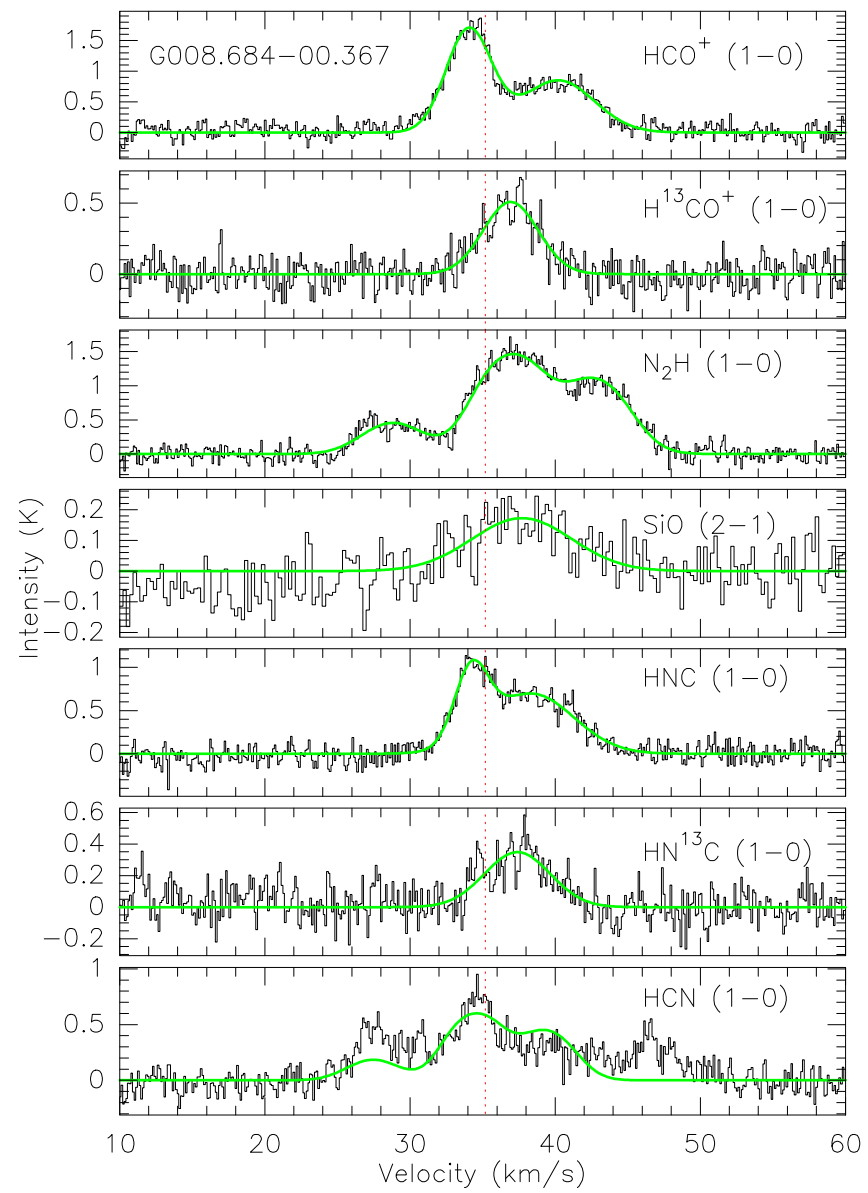

Fig. A.1. Top panels: integrated intensity maps of the $\mathrm{HCO}^{+}(1-0), \mathrm{H}^{13} \mathrm{CO}^{+}(1-0), \mathrm{SiO}, \mathrm{N}_{2} \mathrm{H}^{+}(1-0), \mathrm{HNC}(1-0), \mathrm{HN}^{13} \mathrm{C}(1-0)$ and $\mathrm{HCN}(1-0)$ emission for the high-mass ATLASGAL clumps. The last panel shows the zero moment map contours of the $\mathrm{HCO}^{+}$(black) and $\mathrm{N}_{2} \mathrm{H}^{+}($white) line emissions overlaid on the ATLASGAL submm continuum emission at $870 \mu \mathrm{m}$. Contour levels are shown on each image. The Mopra telescope beam size is shown on the left corner of the maps. Bottom panels: $\mathrm{HCO}^{+}, \mathrm{H}^{13} \mathrm{CO}^{+}, \mathrm{N}_{2} \mathrm{H}^{+}, \mathrm{SiO}, \mathrm{HNC}, \mathrm{HN}{ }^{13} \mathrm{C}, \mathrm{HCN}$ spectra for all sources. Velocity range is $50 \mathrm{~km} \mathrm{~s}^{-1}$. The spectra is created by averaging the spectra in $38^{\prime \prime} \times 38^{\prime \prime}$ box (MOPRA beam size) around the clump's $\mathrm{N}_{2} \mathrm{H}^{+}$peak emission. The vertical red dotted line indicates the radial velocity of the ammonia line for the clumps given in Wienen et al. (2015). The single and double Gaussian or hyperfine line fits are shown with green lines. 
A\&A 620, A158 (2018)
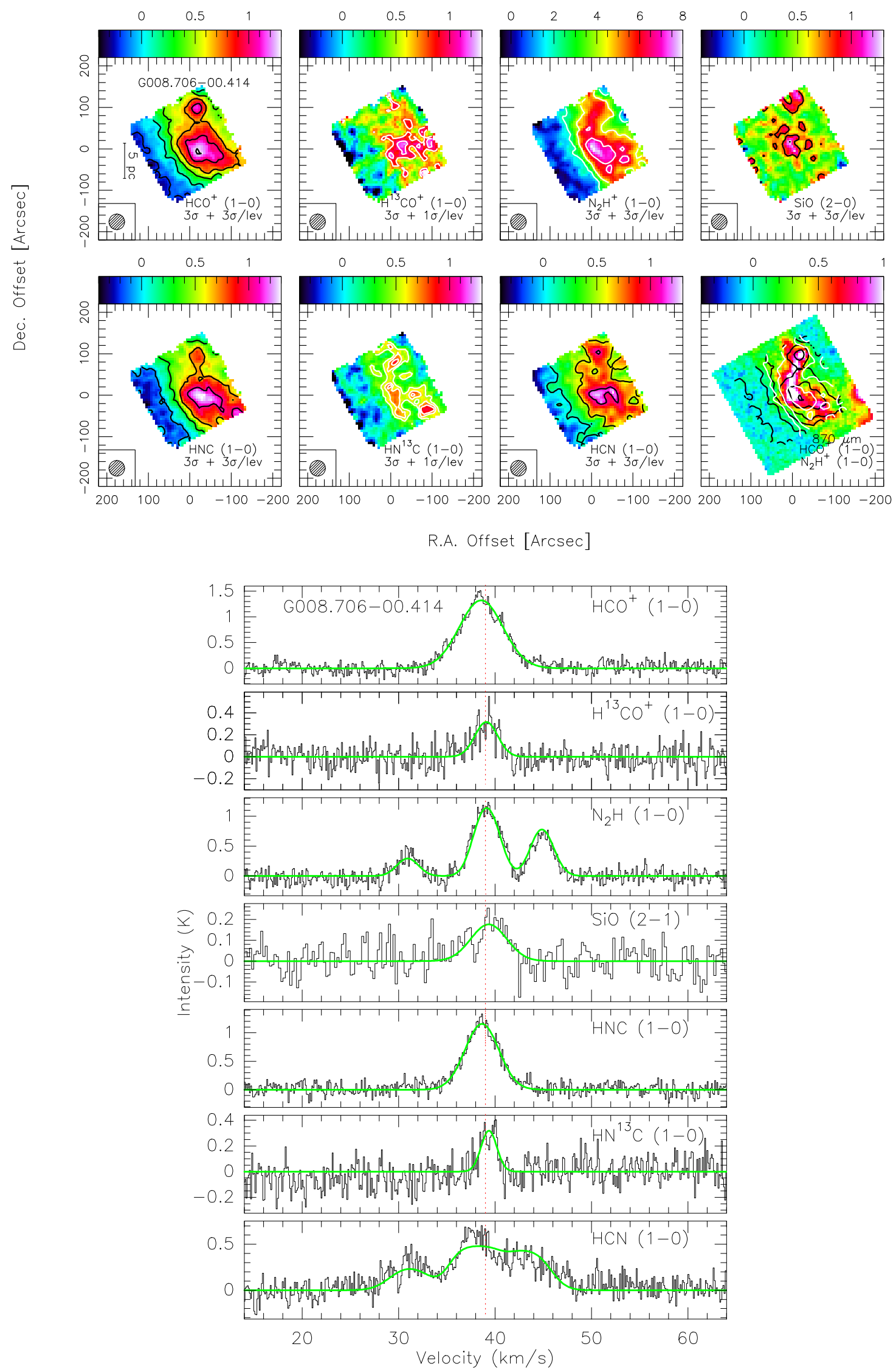

Fig. A.1. continued. 
G. Saral et al.: MALT90 molecular content on high-mass IR-dark clumps
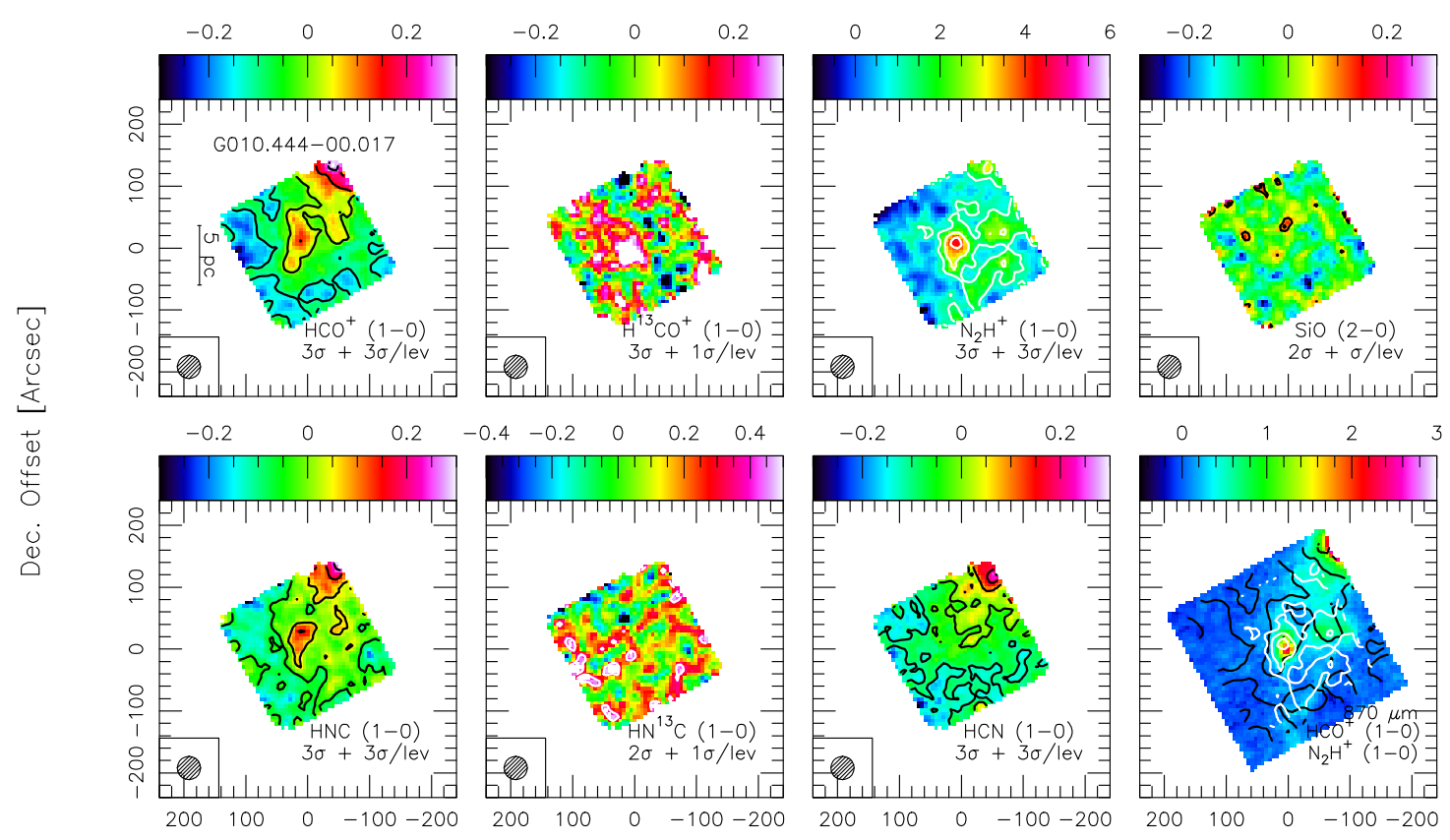

R.A. Offset [Arcsec]

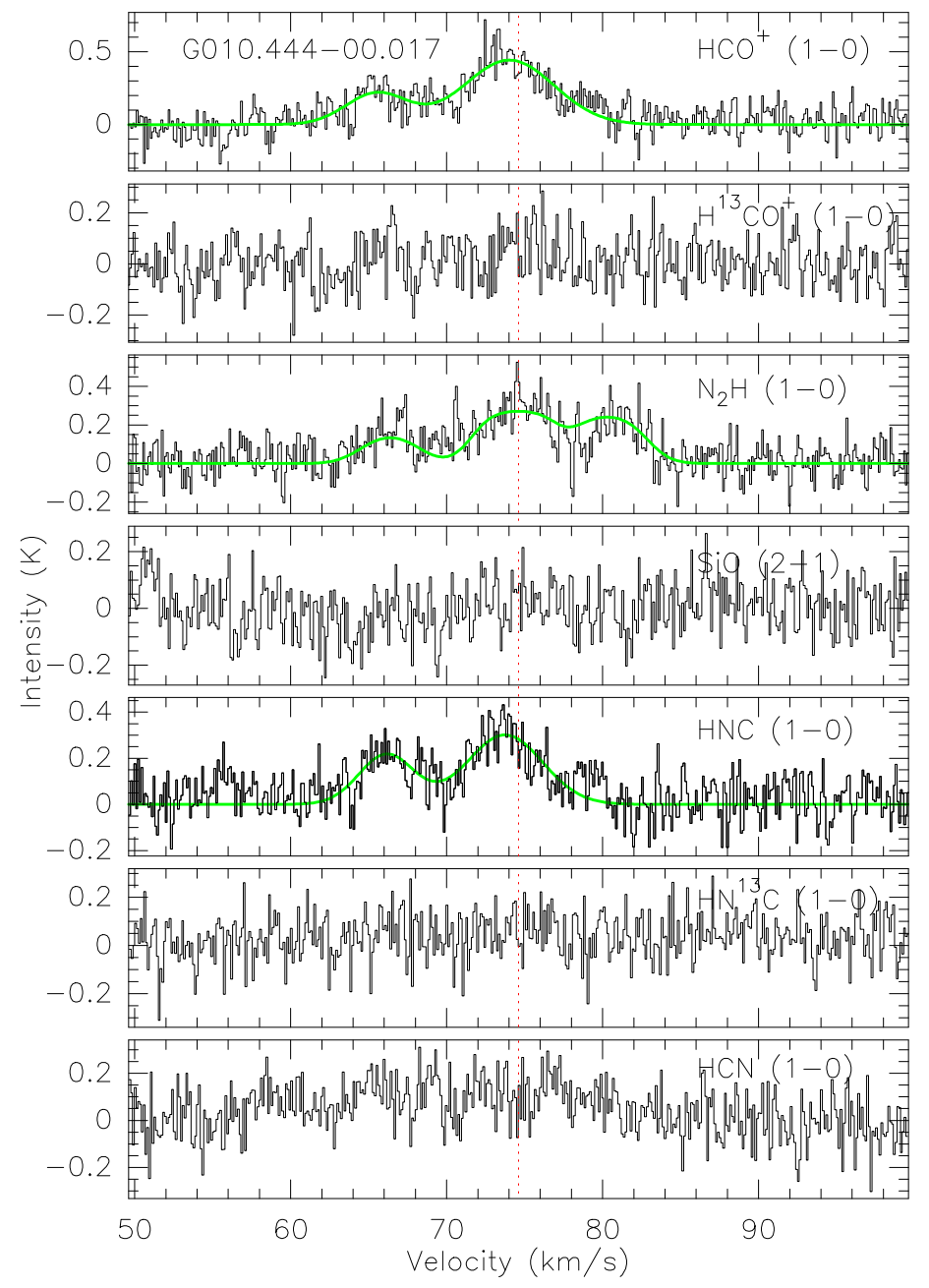

Fig. A.1. continued. 

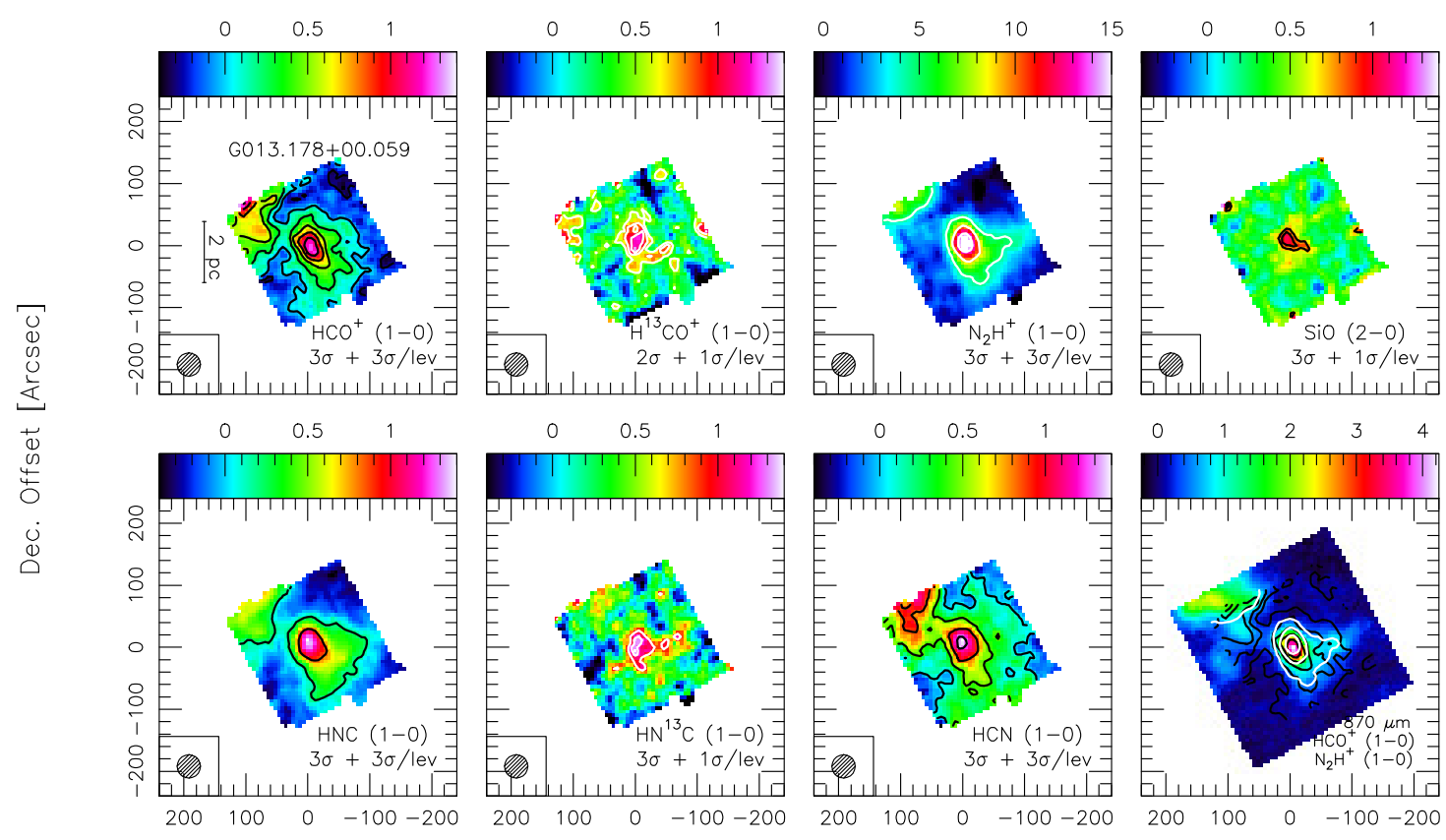

R.A. Offset [Arcsec]

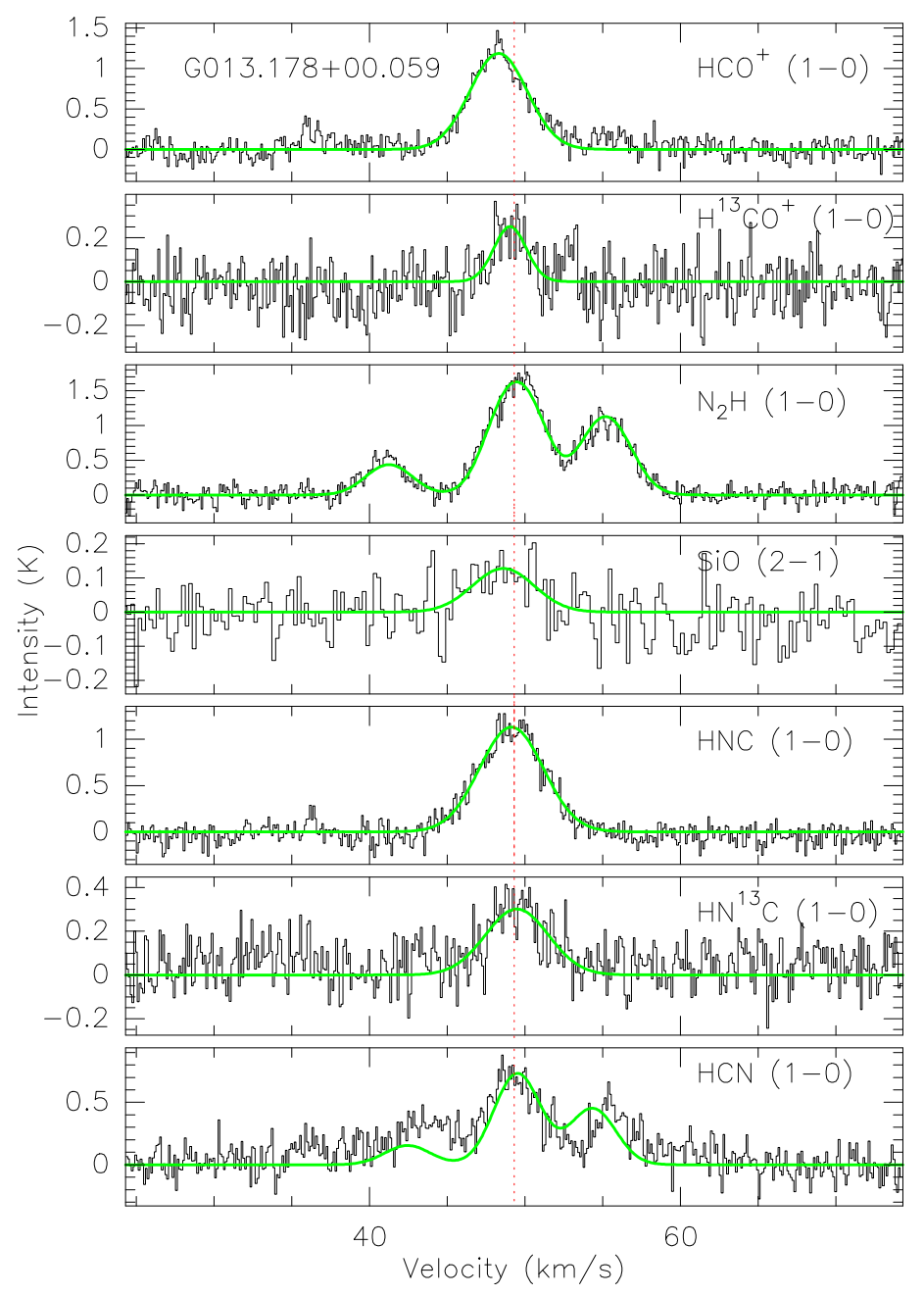

Fig. A.1. continued. 
G. Saral et al.: MALT90 molecular content on high-mass IR-dark clumps
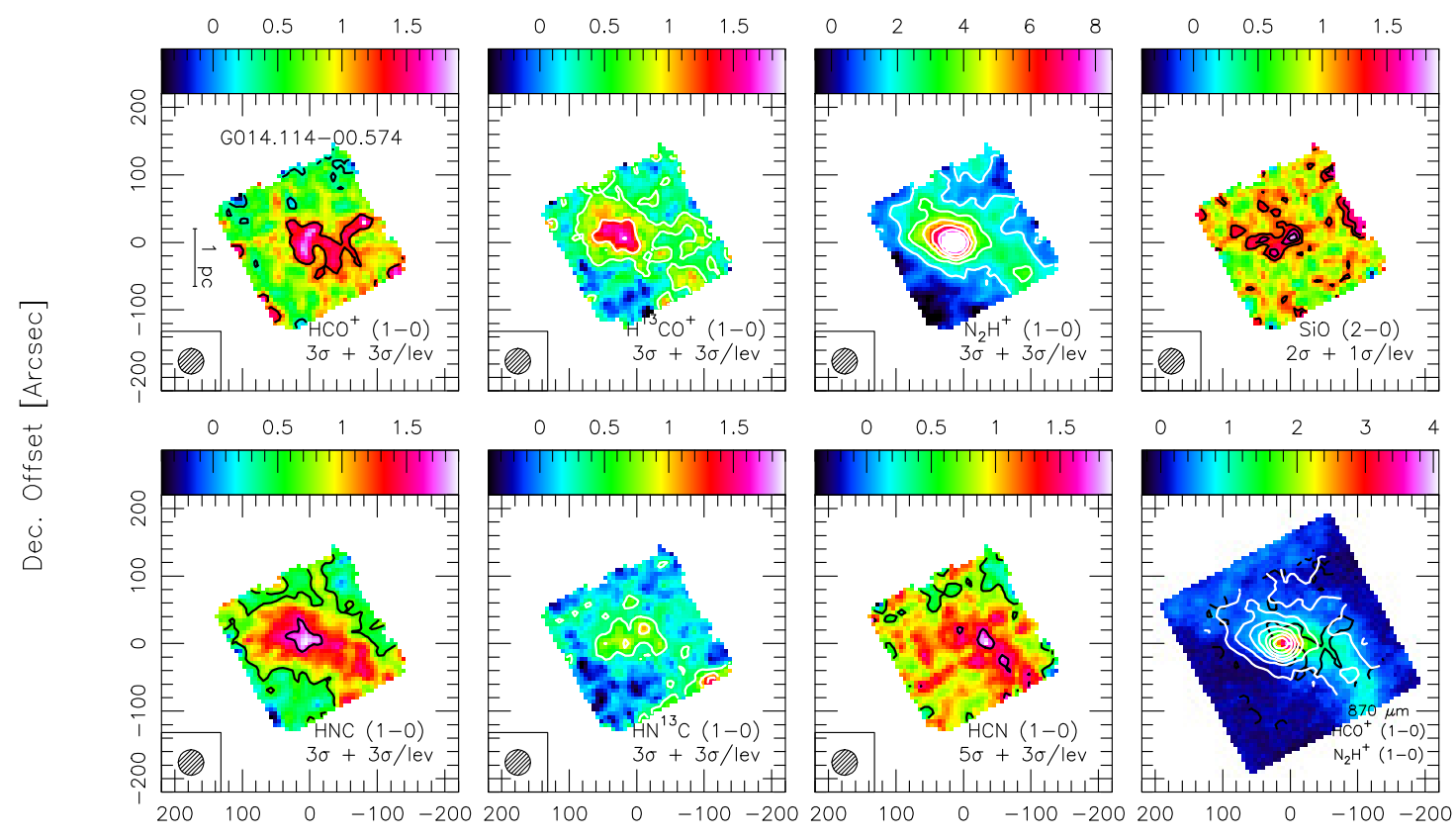

R.A. Offset [Arcsec]

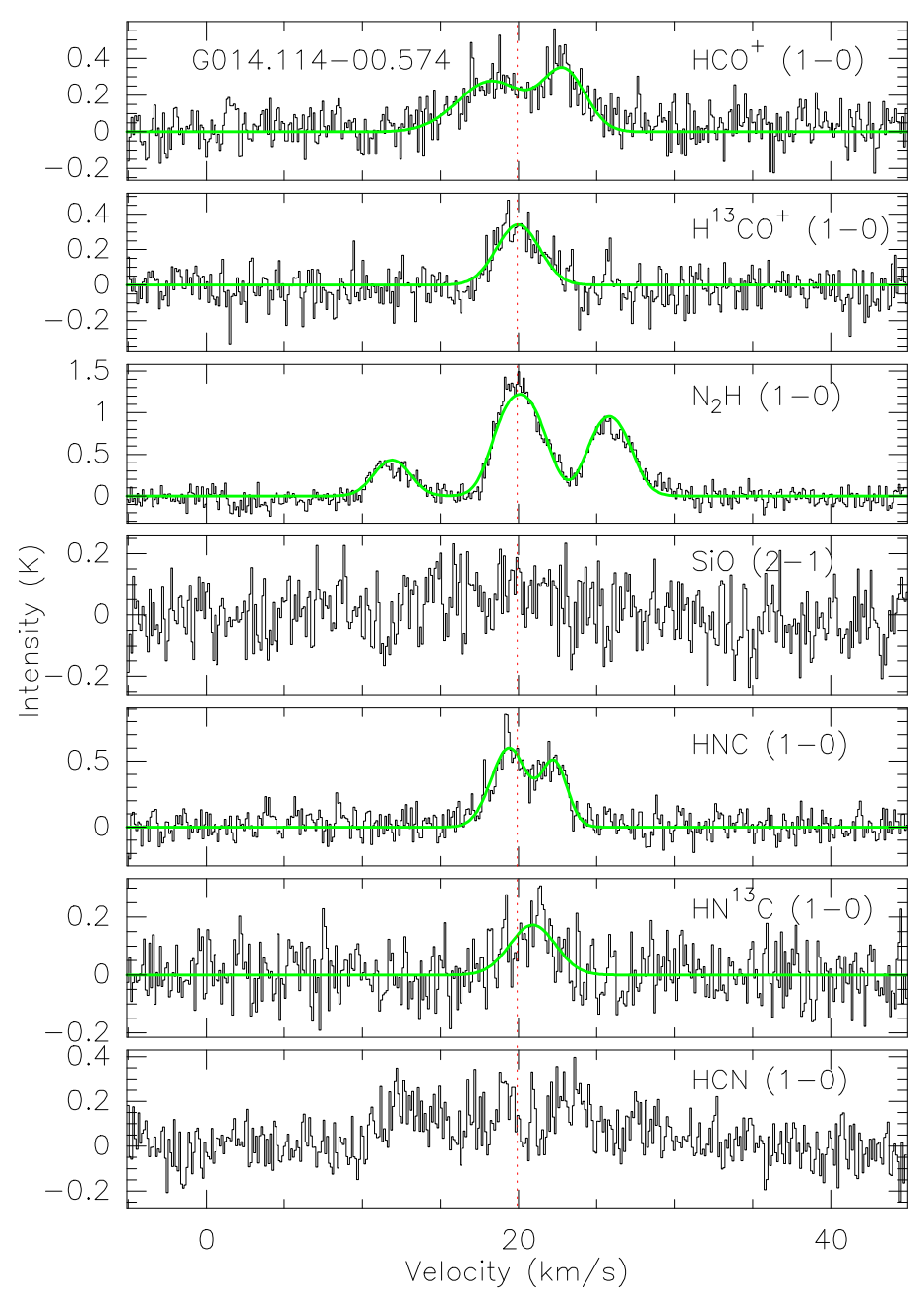

Fig. A.1. continued. 

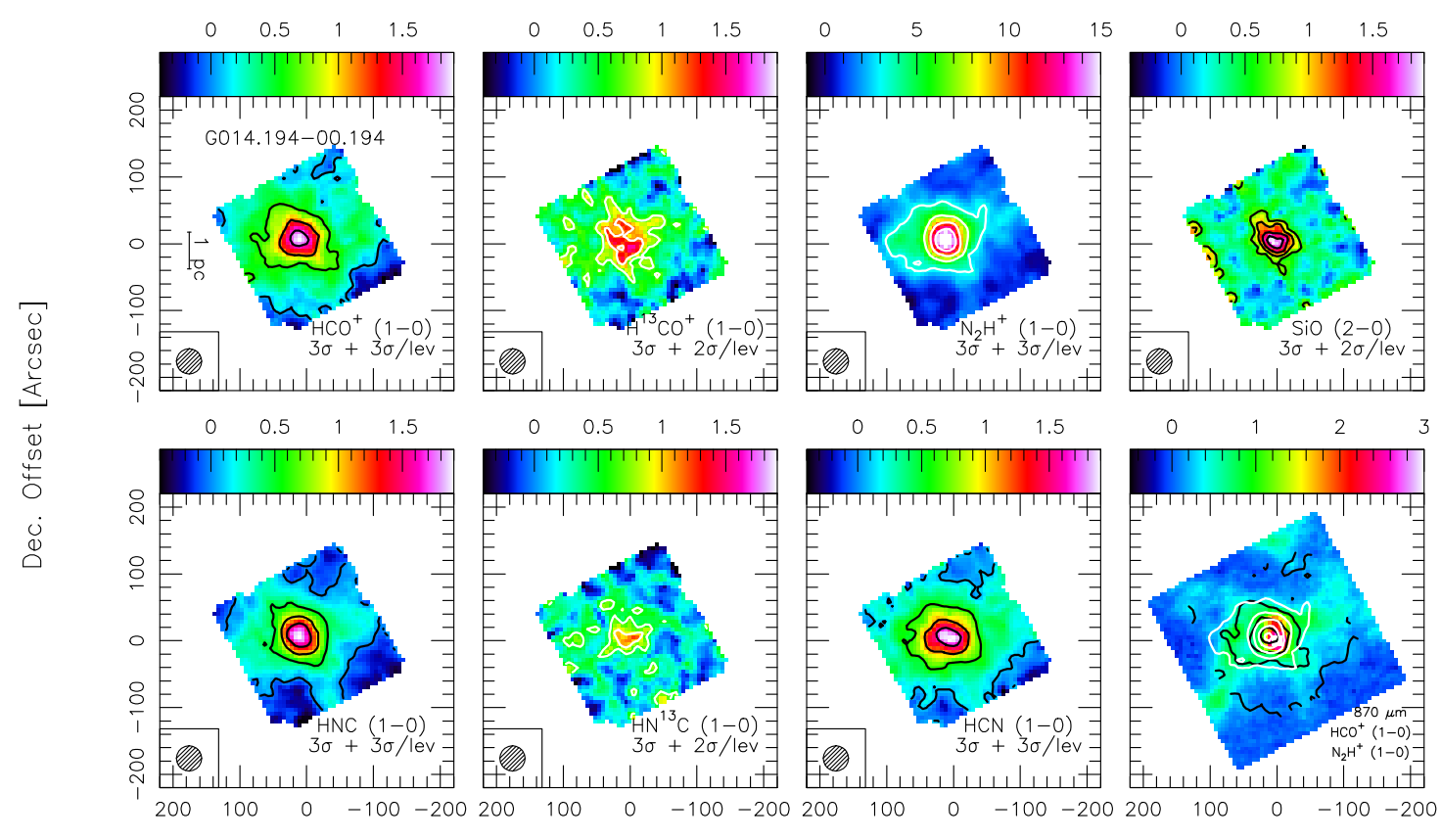

R.A. Offset [Arcsec]

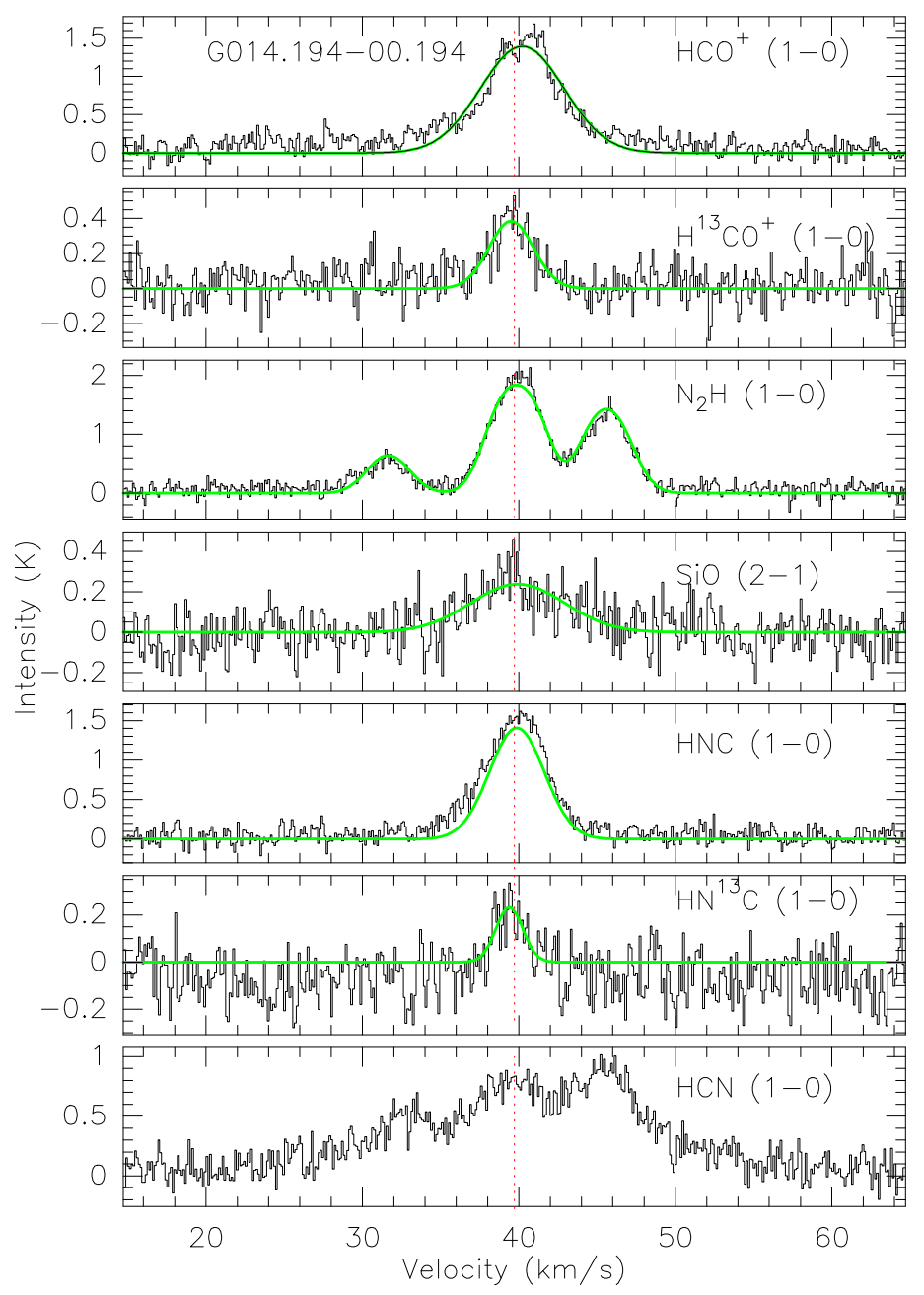

Fig. A.1. continued. 
G. Saral et al.: MALT90 molecular content on high-mass IR-dark clumps
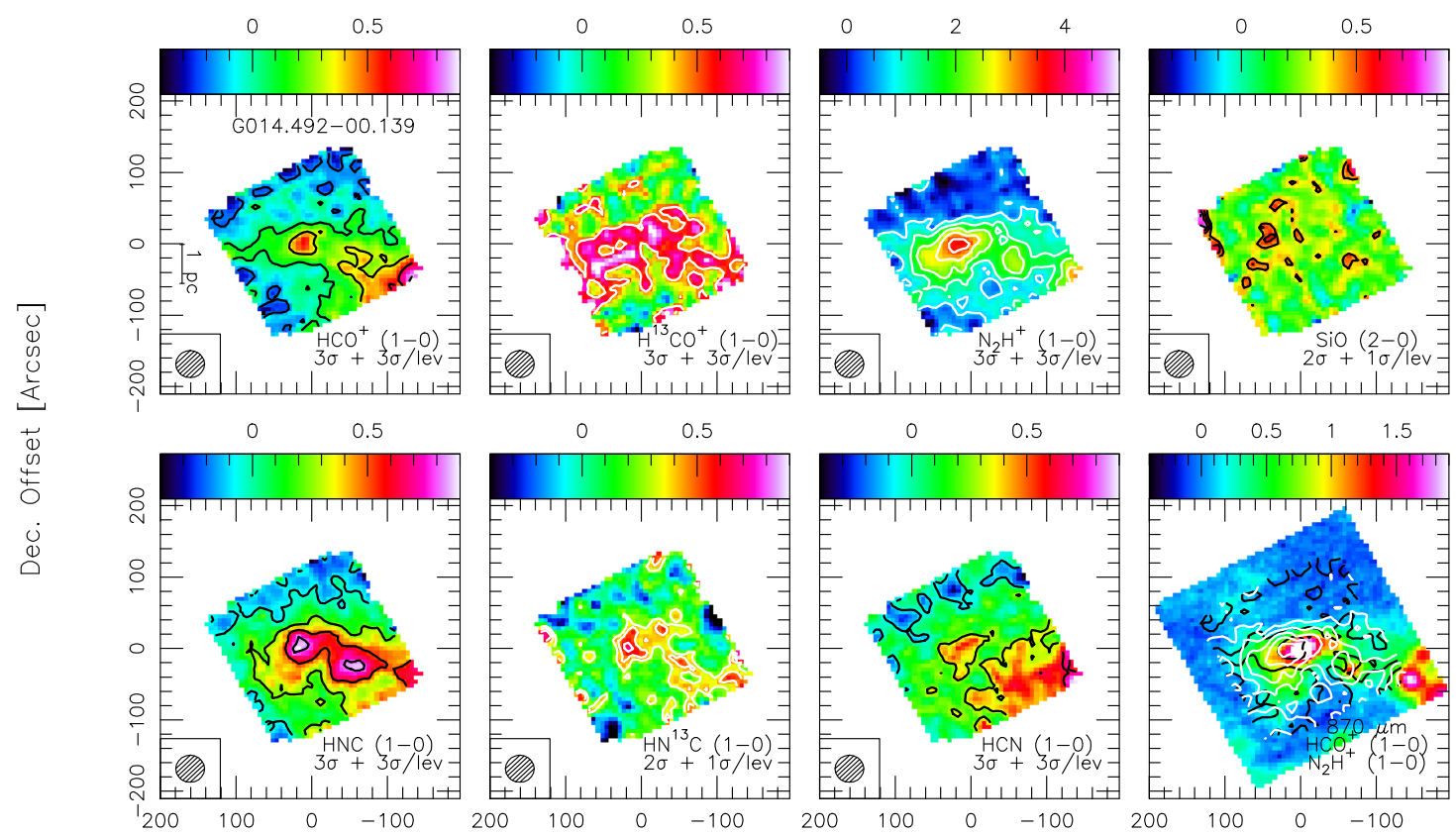

R.A. Offset $[$ Arcsec]

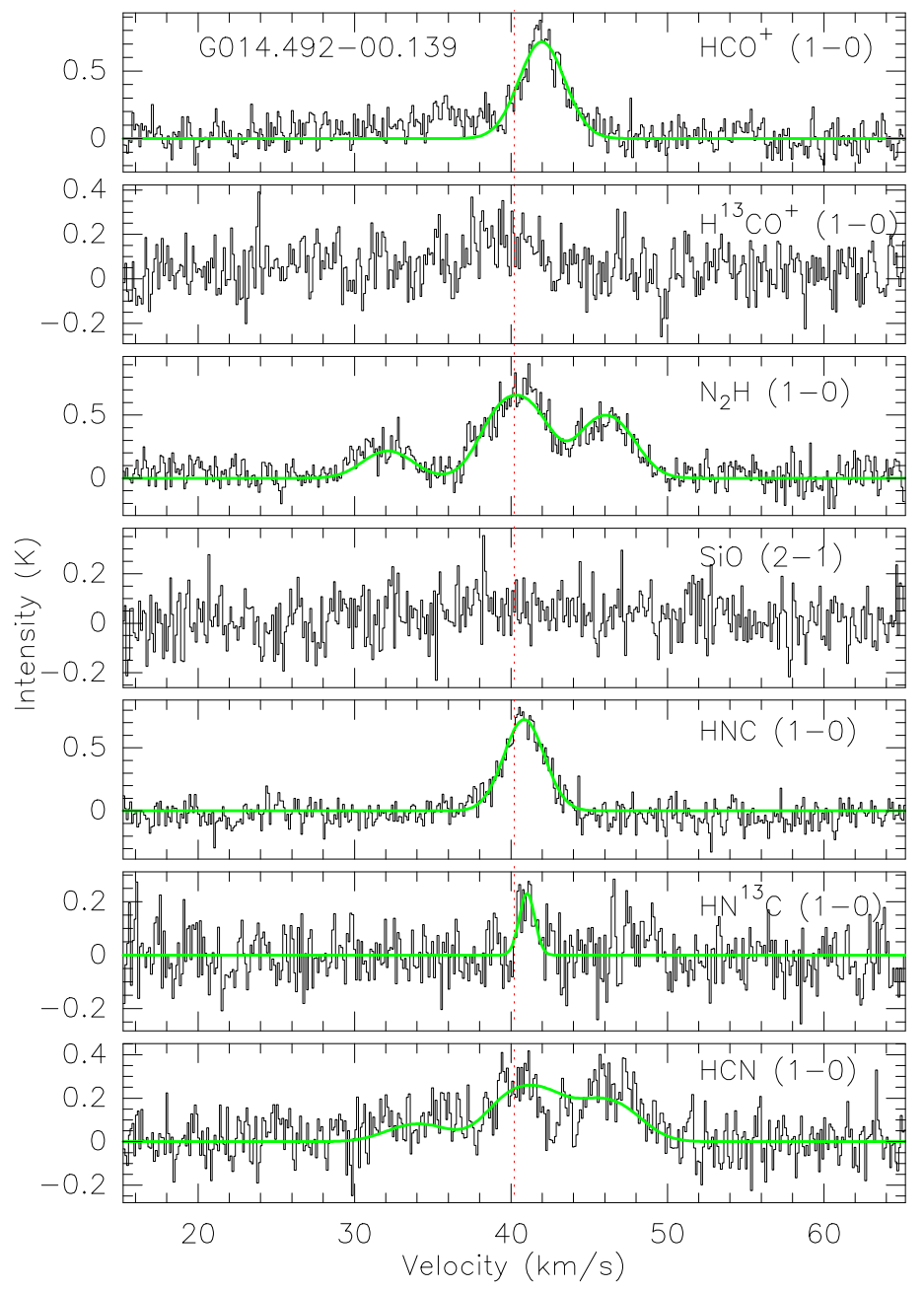

Fig. A.1. continued. 

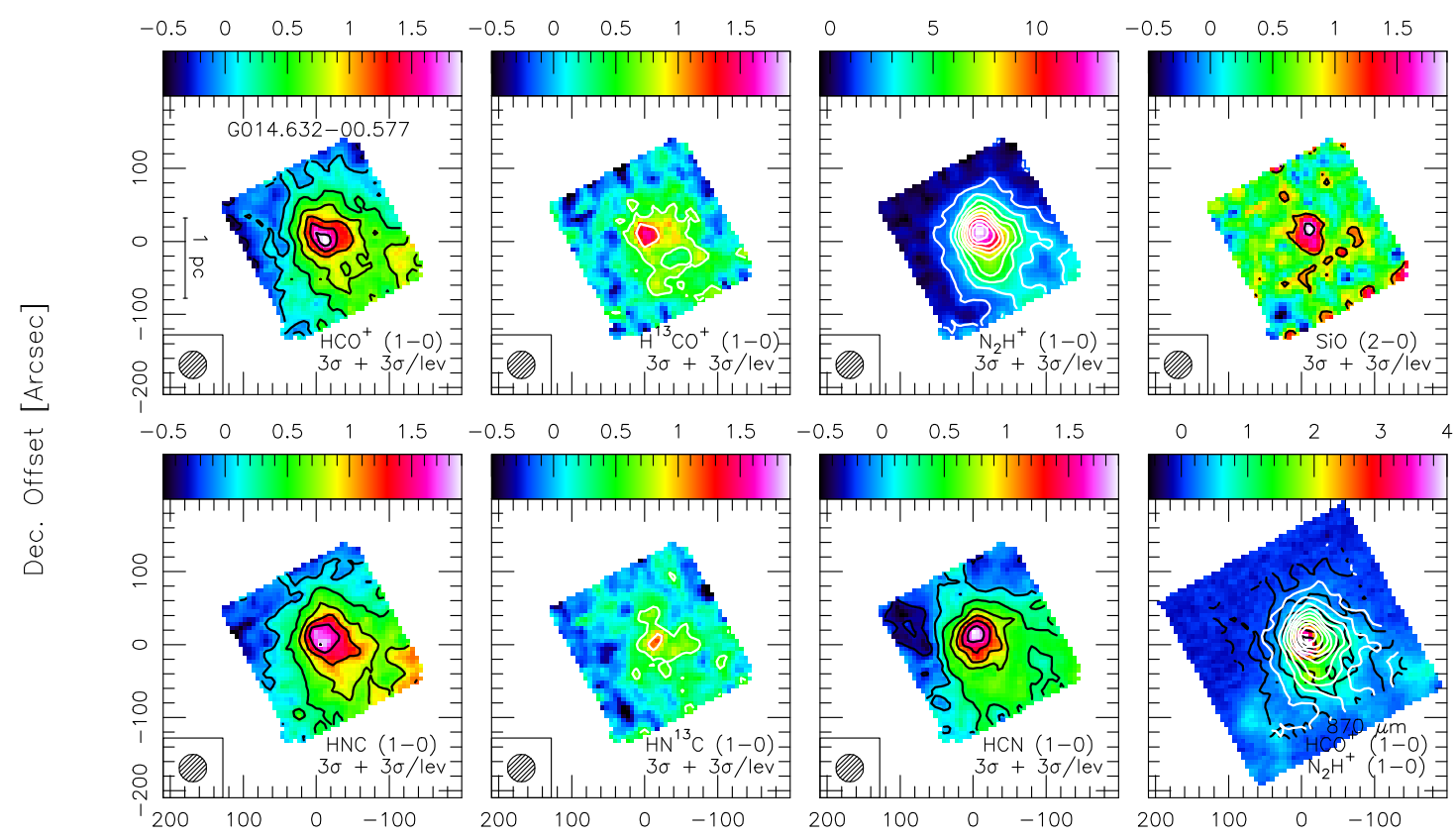

R.A. Offset [Arcsec]

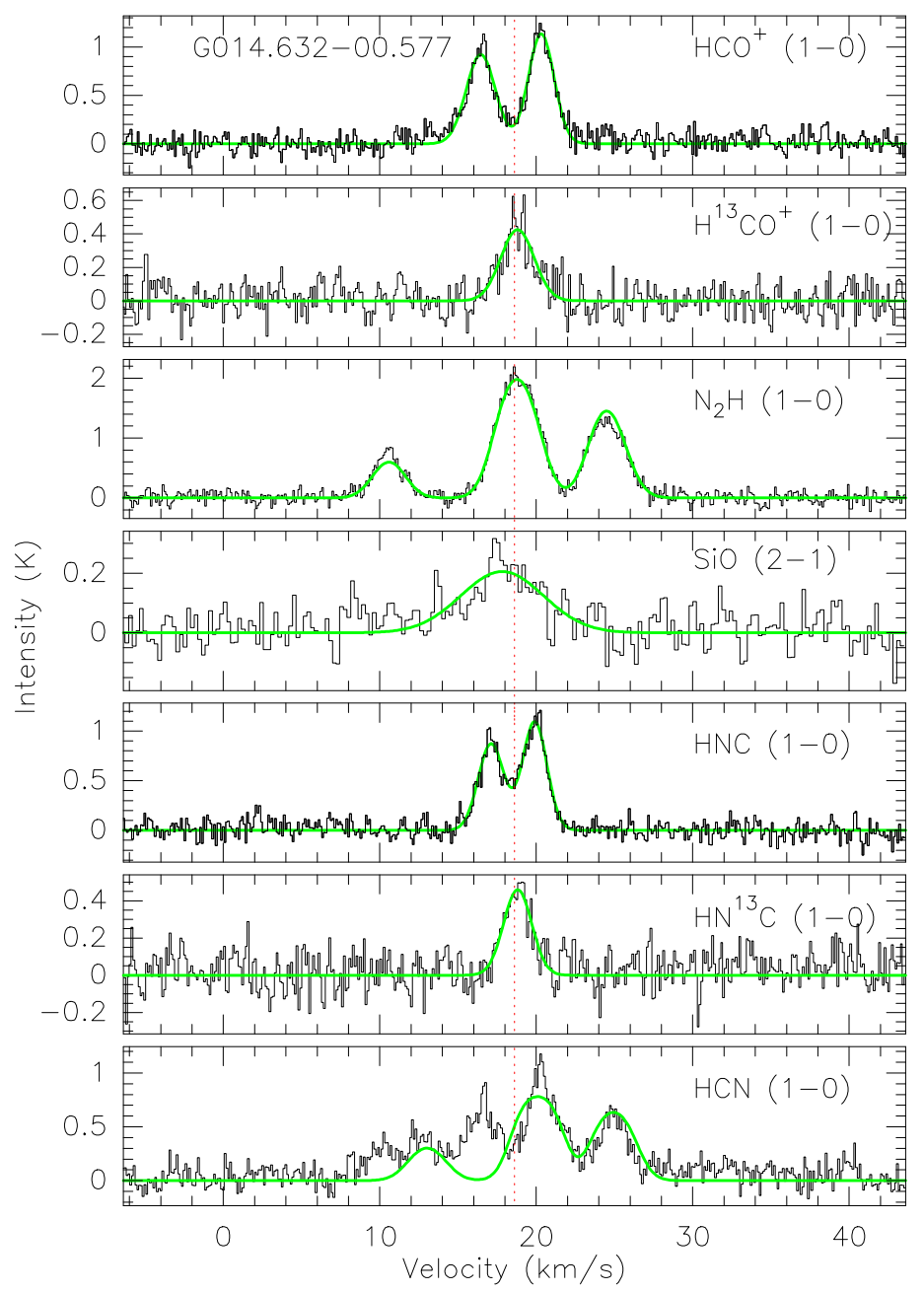

Fig. A.1. continued. 
G. Saral et al.: MALT90 molecular content on high-mass IR-dark clumps
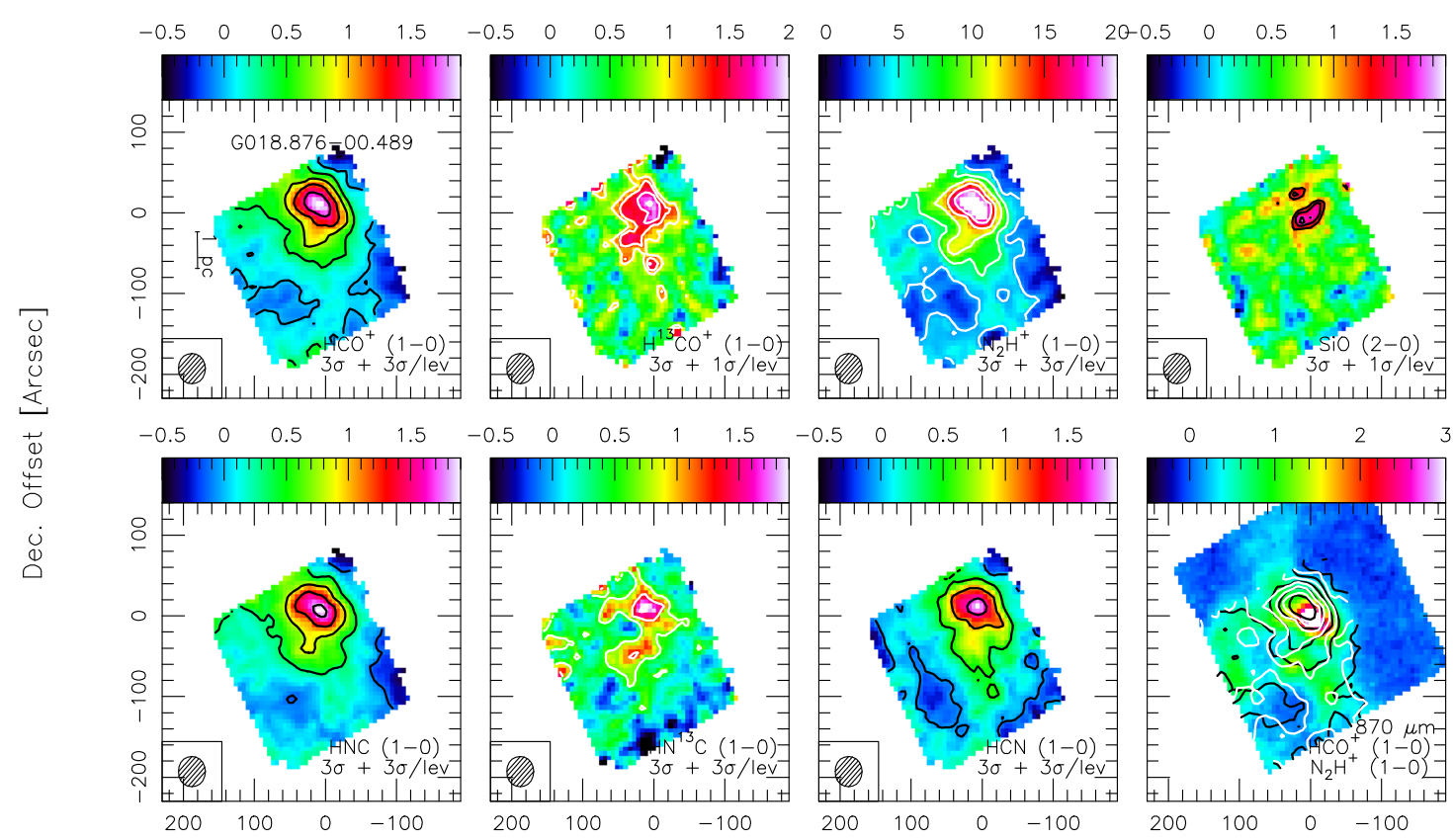

R.A. Offset [Arcsec]

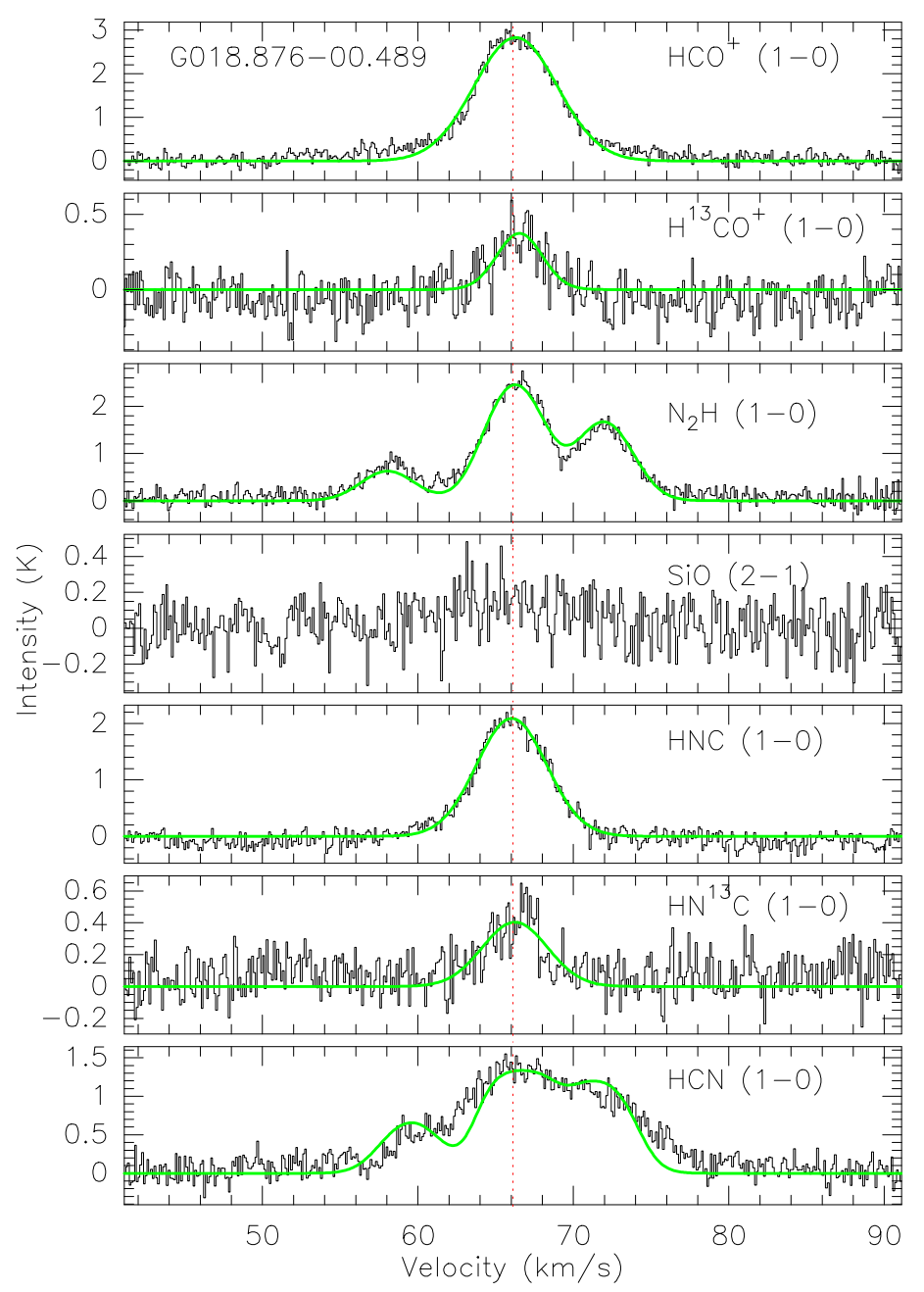

Fig. A.1. continued. 

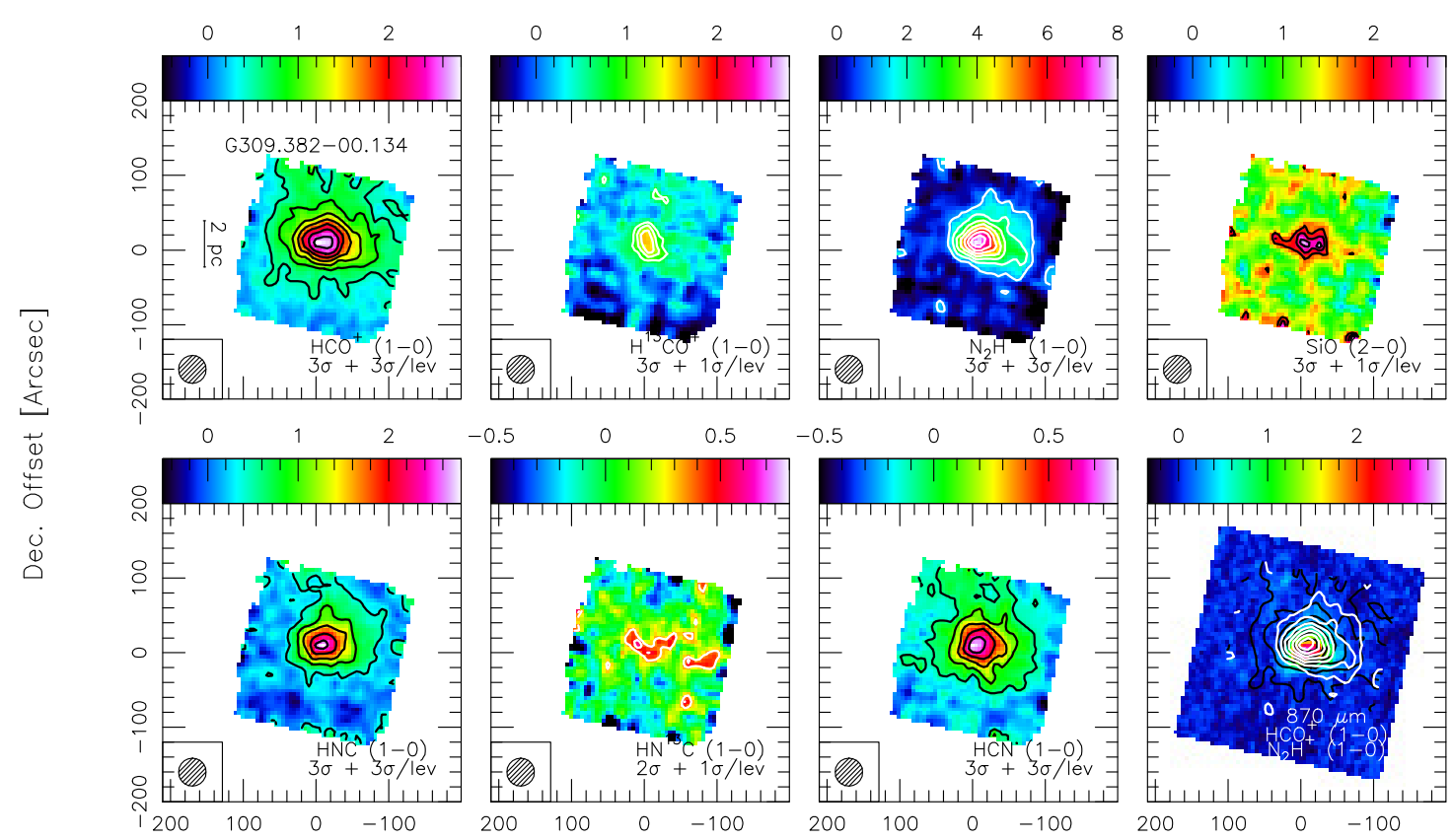

R.A. Offset [Arcsec]

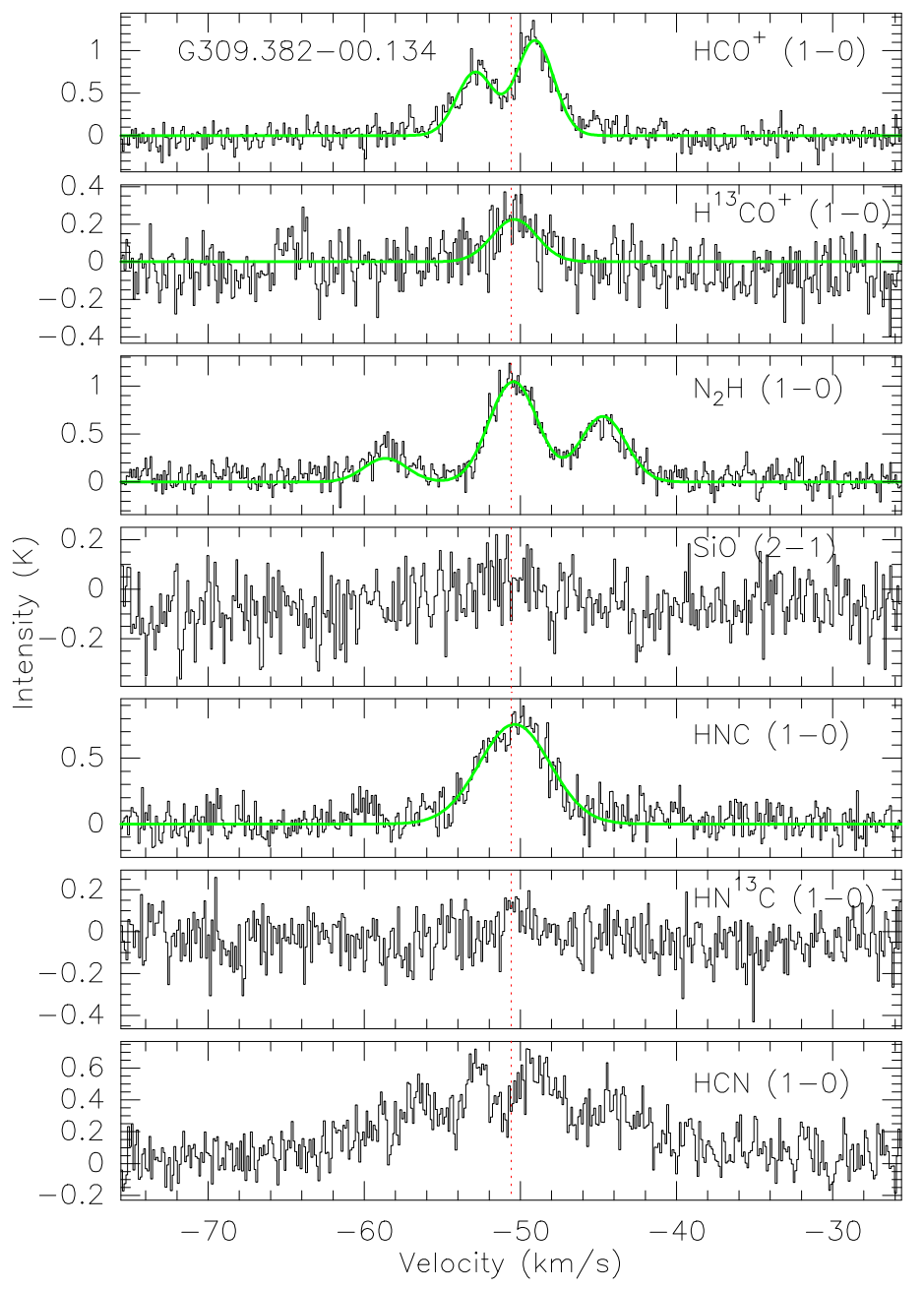

Fig. A.1. continued. 
G. Saral et al.: MALT90 molecular content on high-mass IR-dark clumps
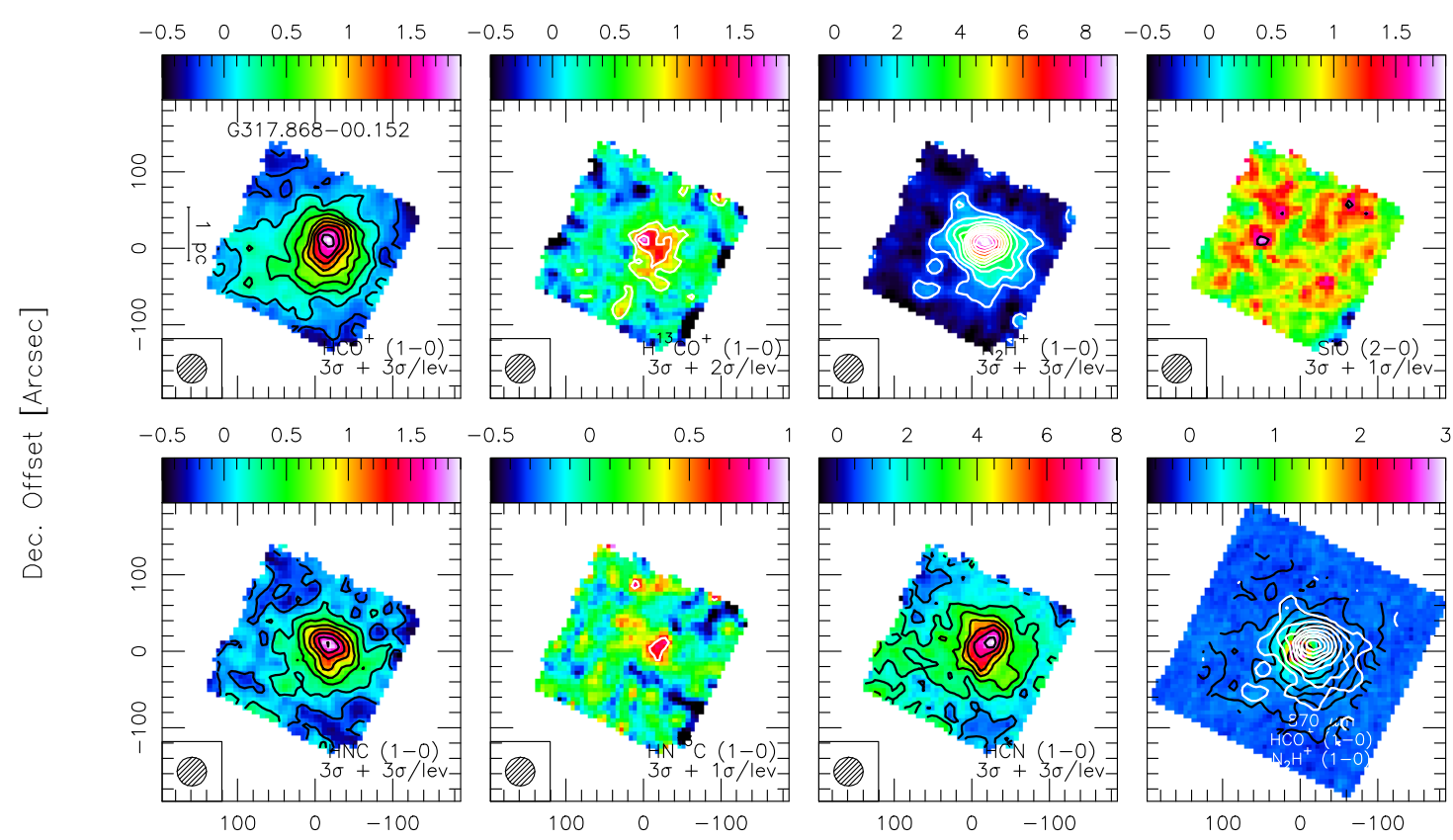

R.A. Offset [Arcsec]

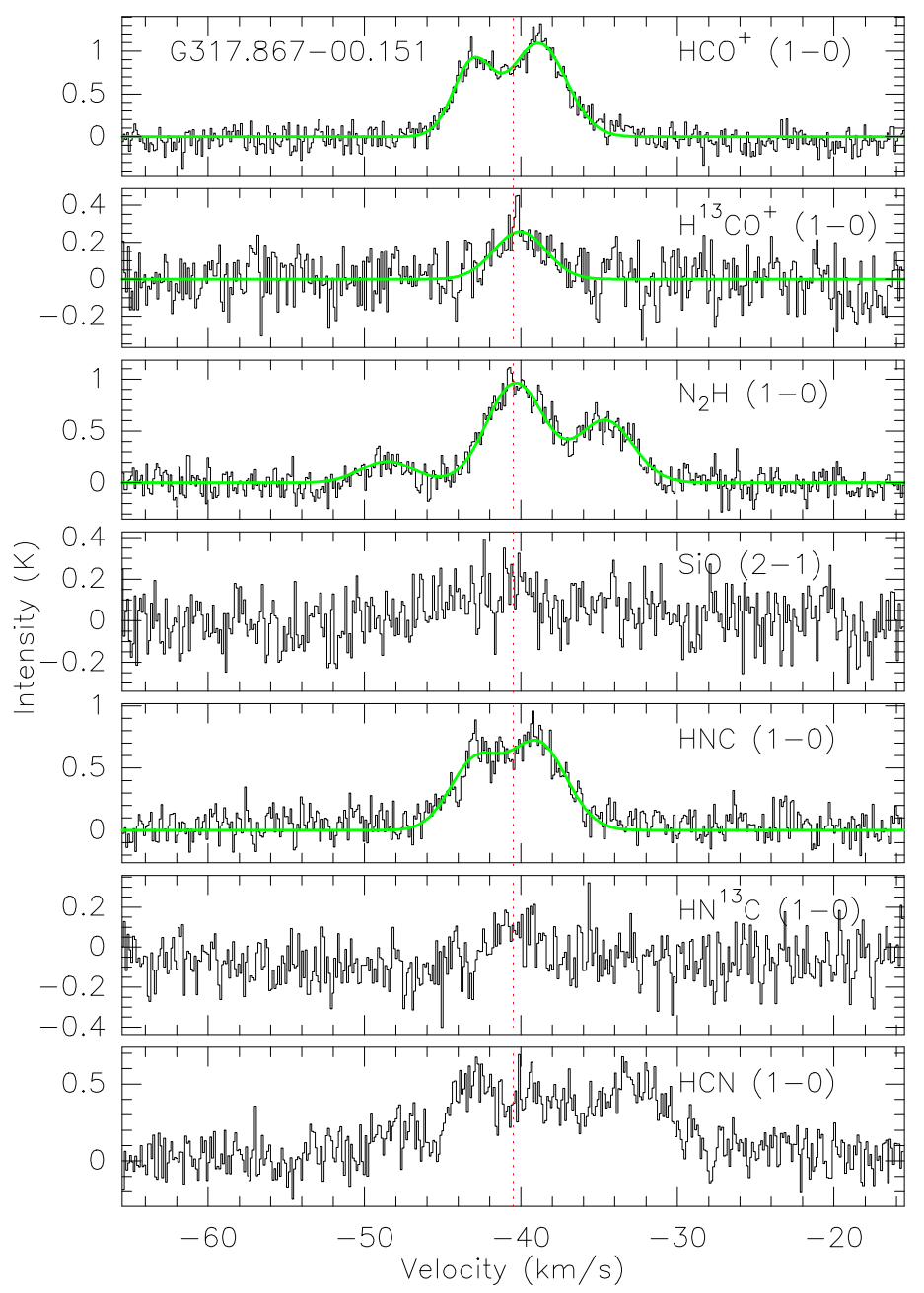

Fig. A.1. continued. 

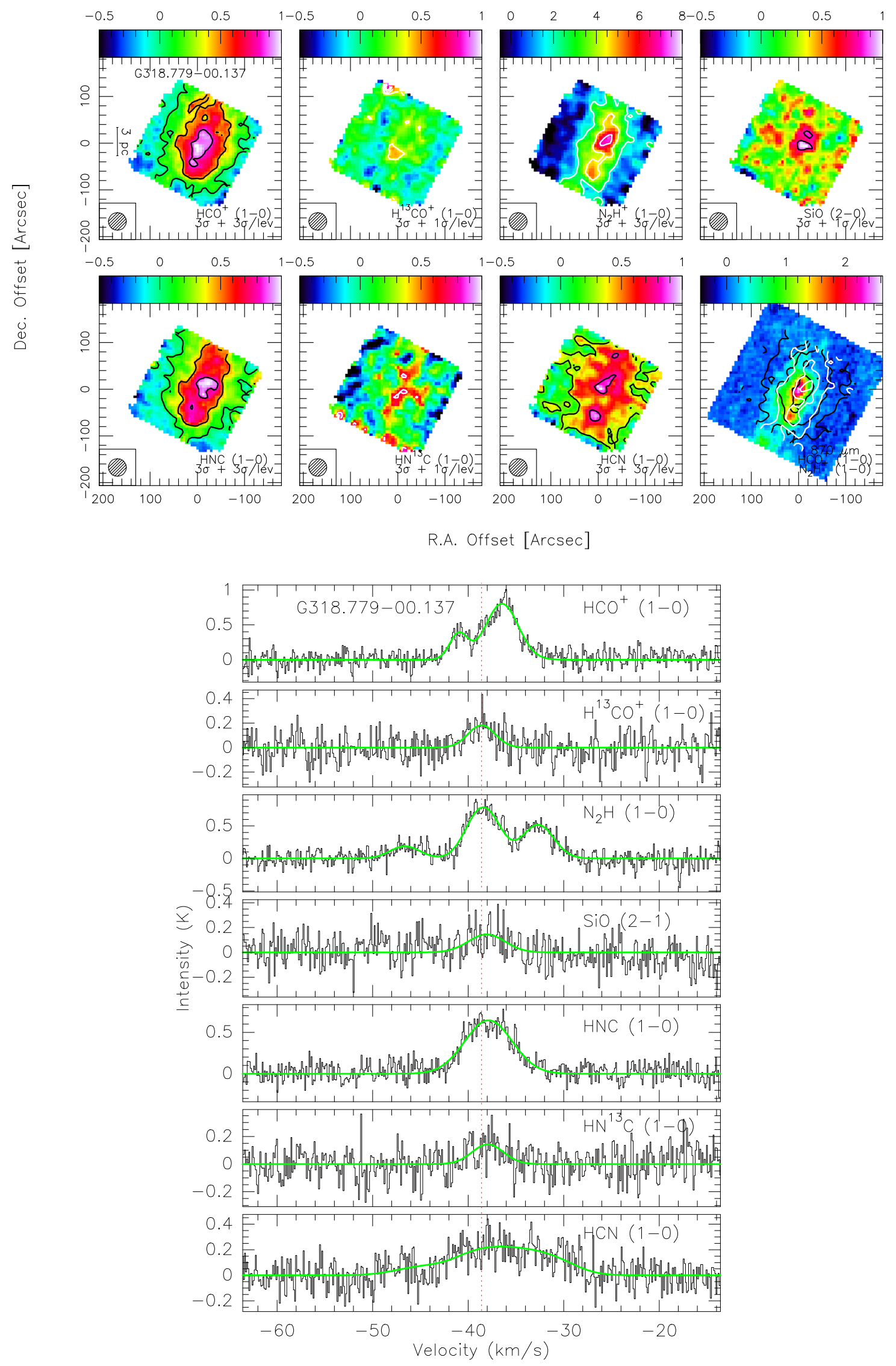

Fig. A.1. continued. 
G. Saral et al.: MALT90 molecular content on high-mass IR-dark clumps
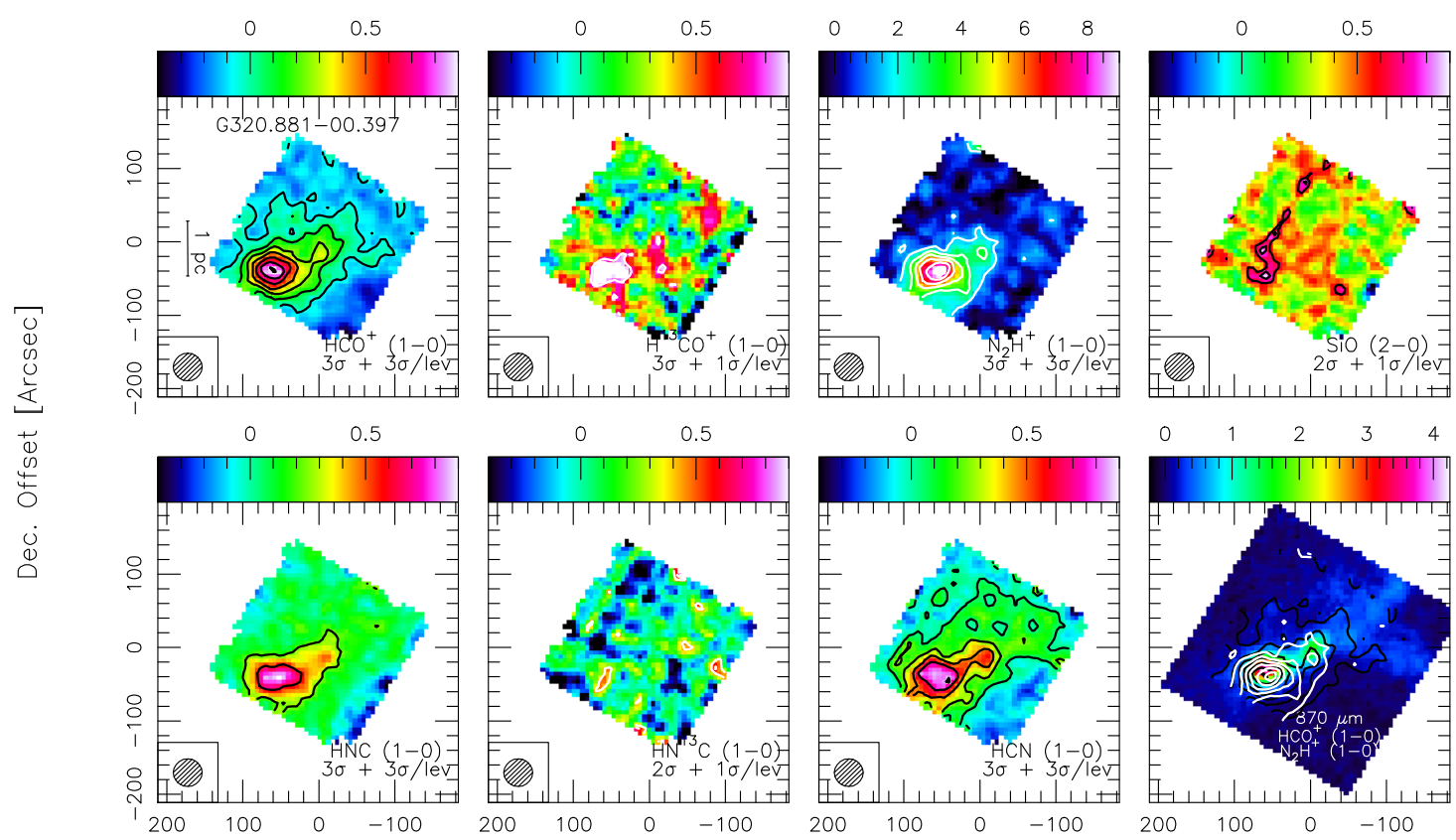

R.A. Offset [Arcsec]

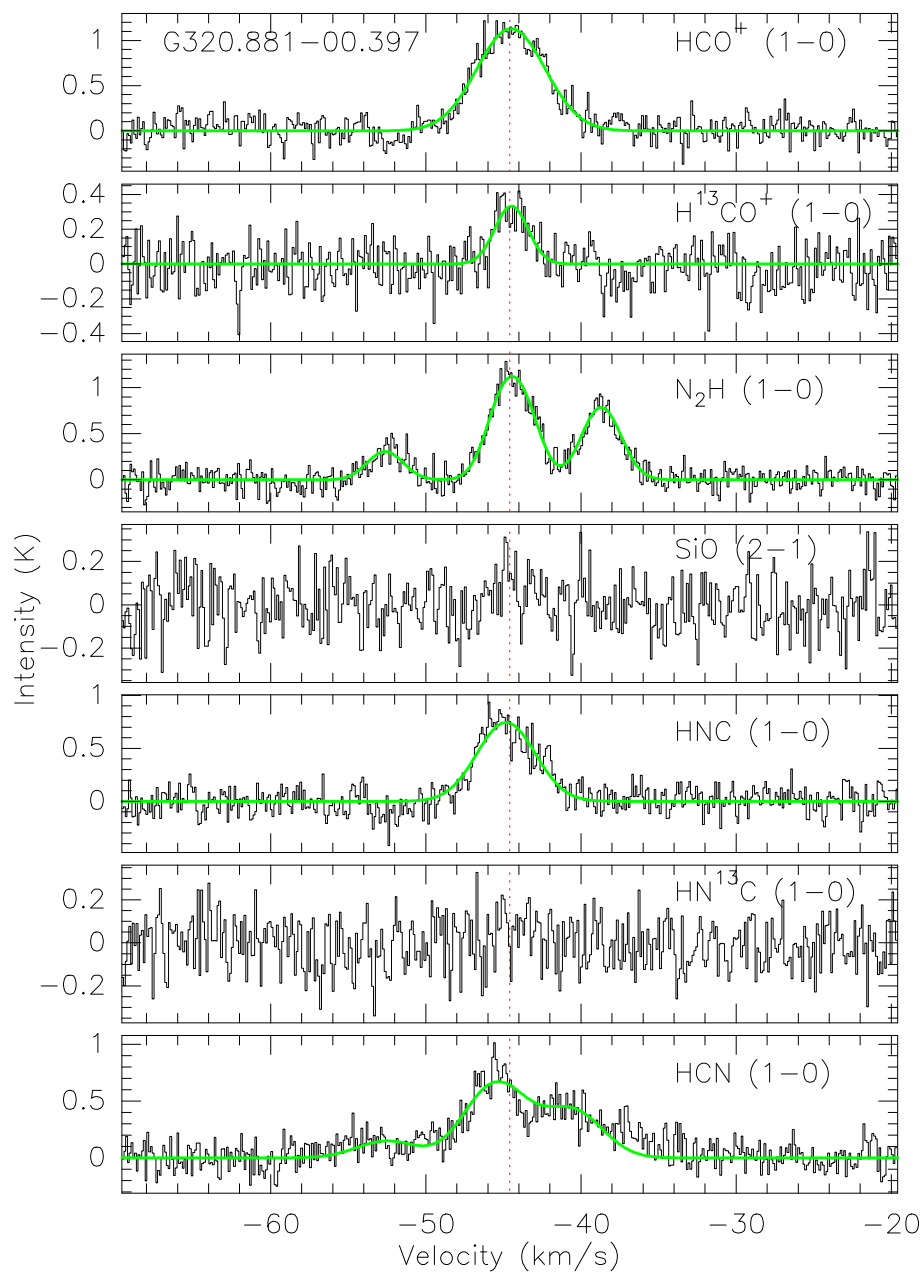

Fig. A.1. continued. 

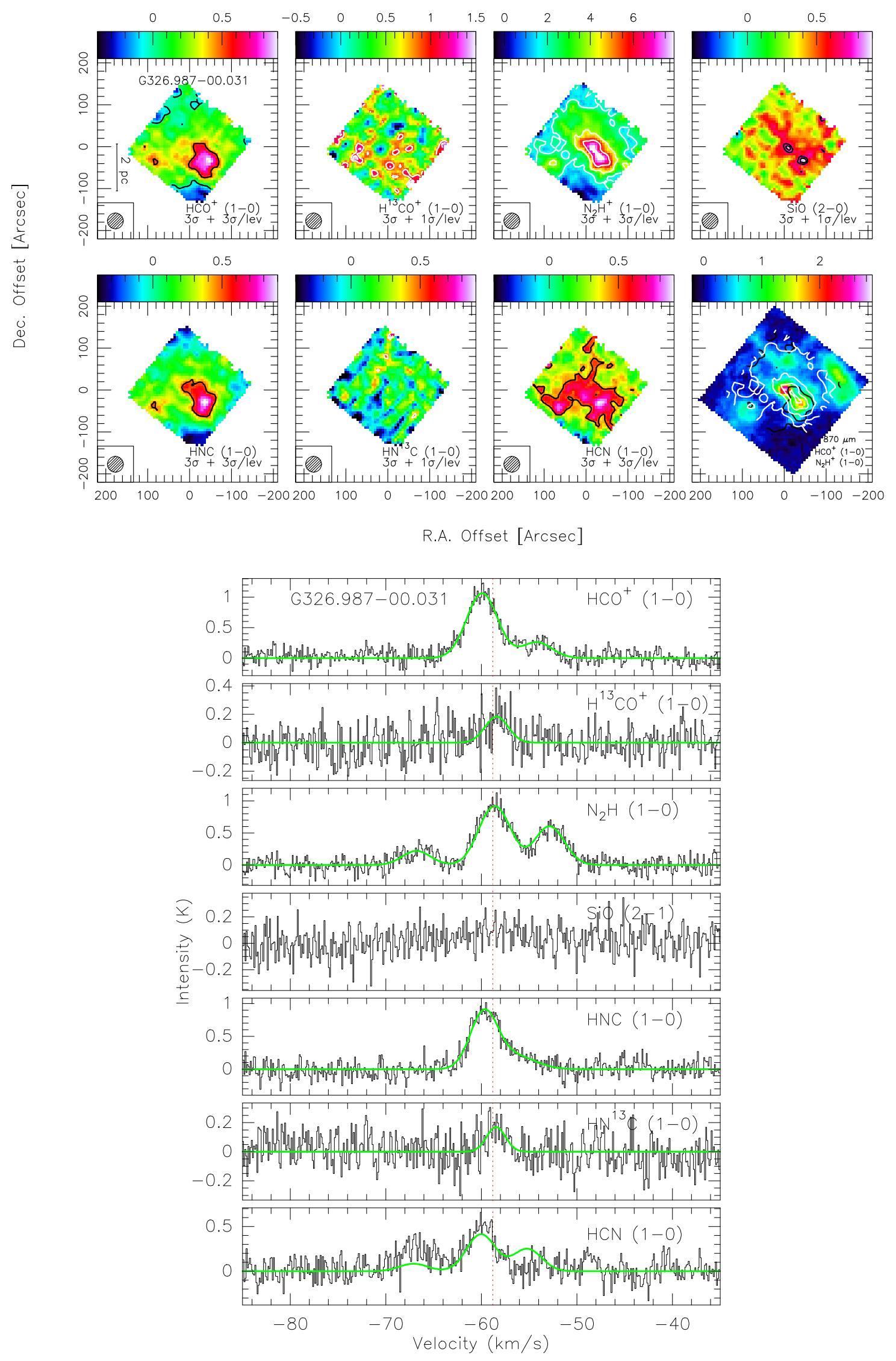

Fig. A.1. continued. 
G. Saral et al.: MALT90 molecular content on high-mass IR-dark clumps
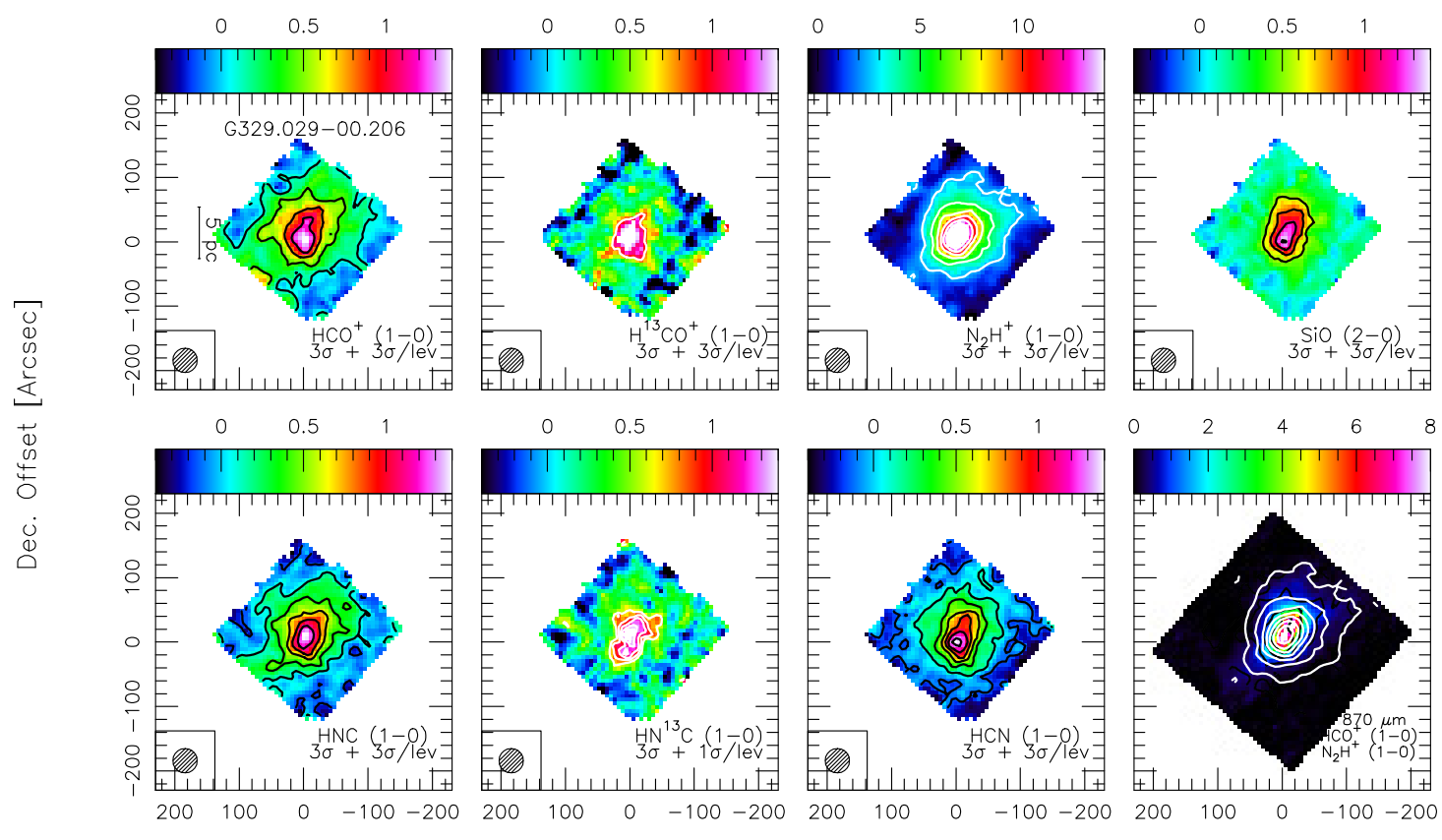

R.A. Offset [Arcsec]

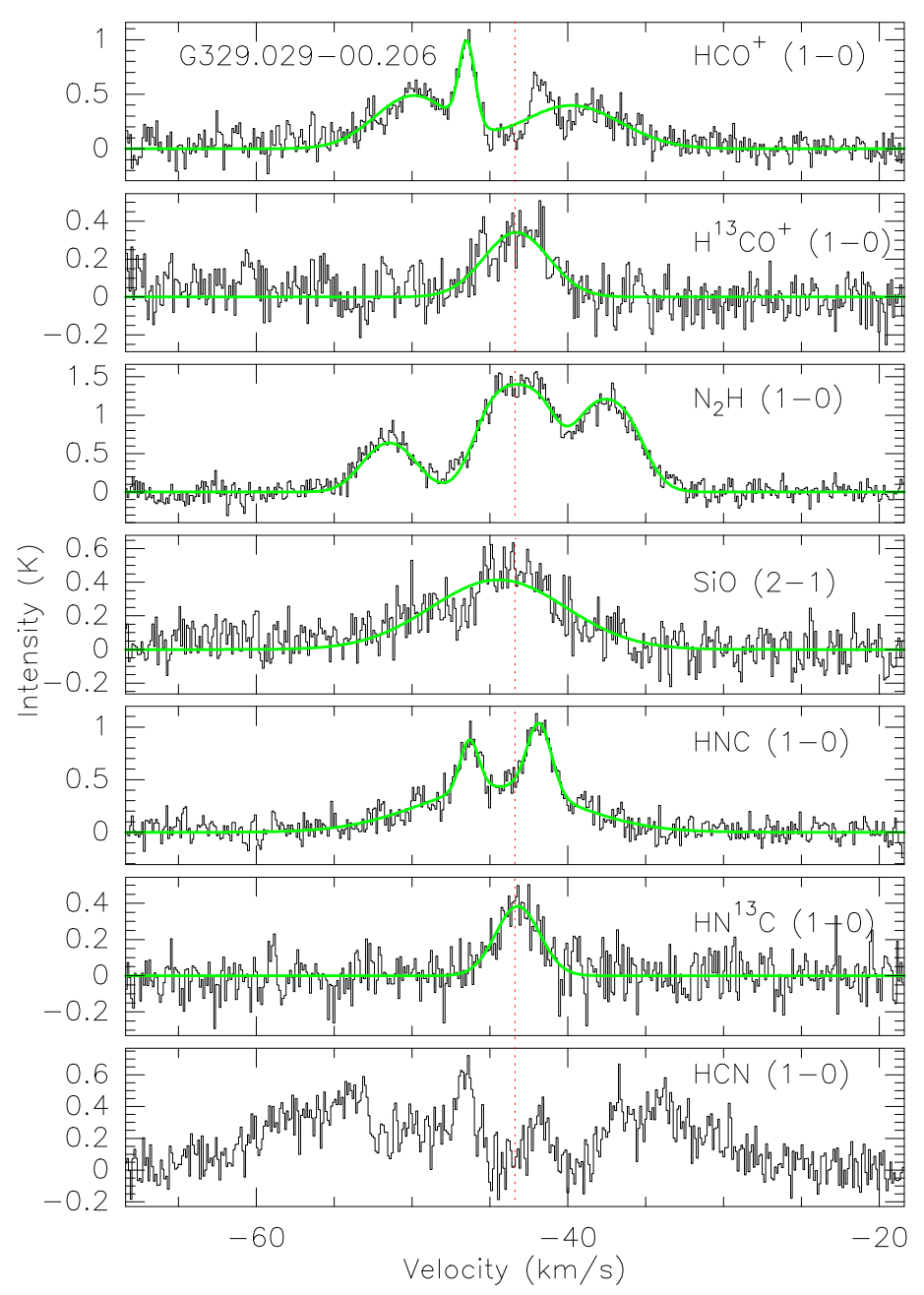

Fig. A.1. continued. 

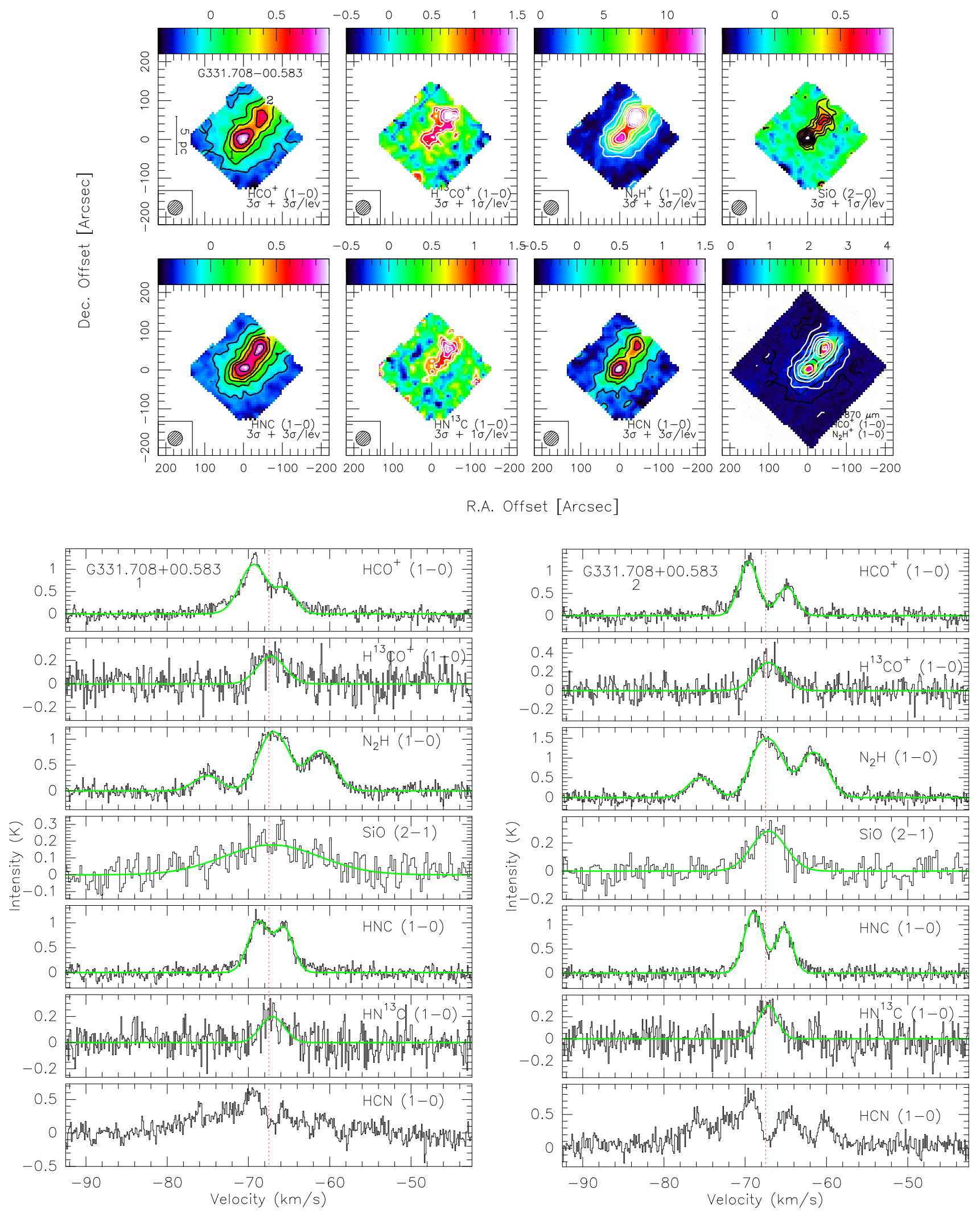

Fig. A.1. continued. 
G. Saral et al.: MALT90 molecular content on high-mass IR-dark clumps
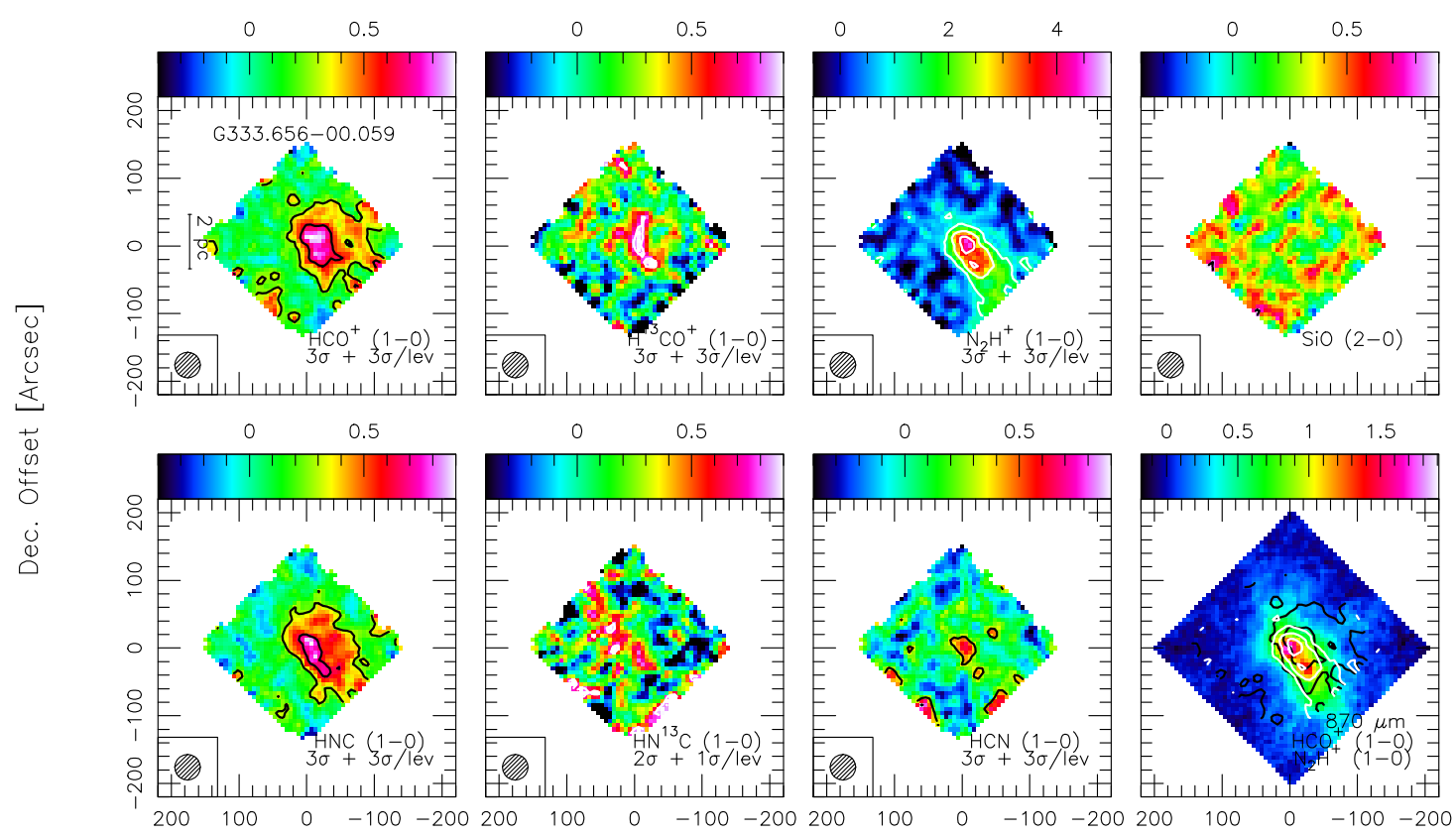

R.A. Offset [Arcsec]

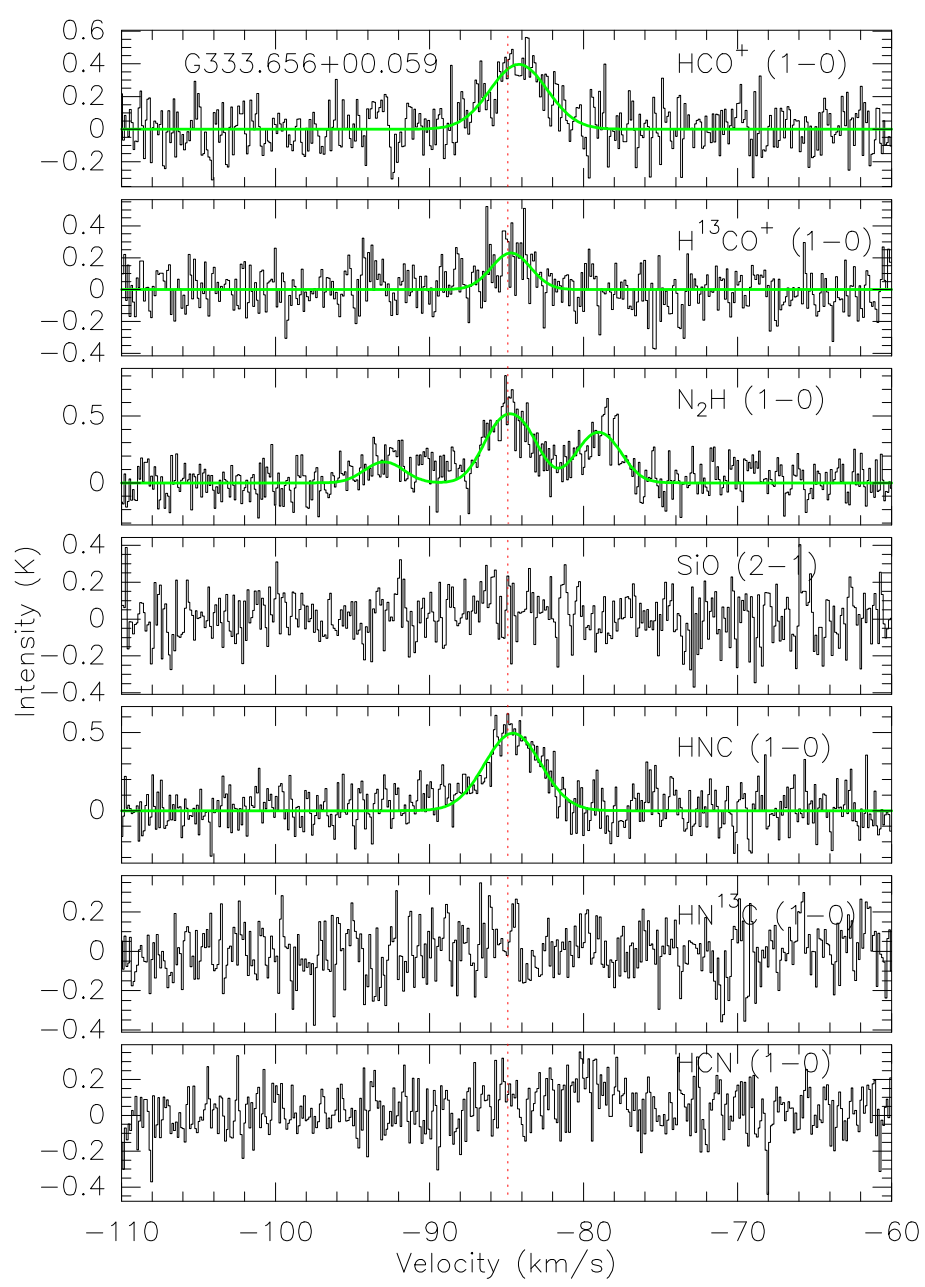

Fig. A.1. continued. 

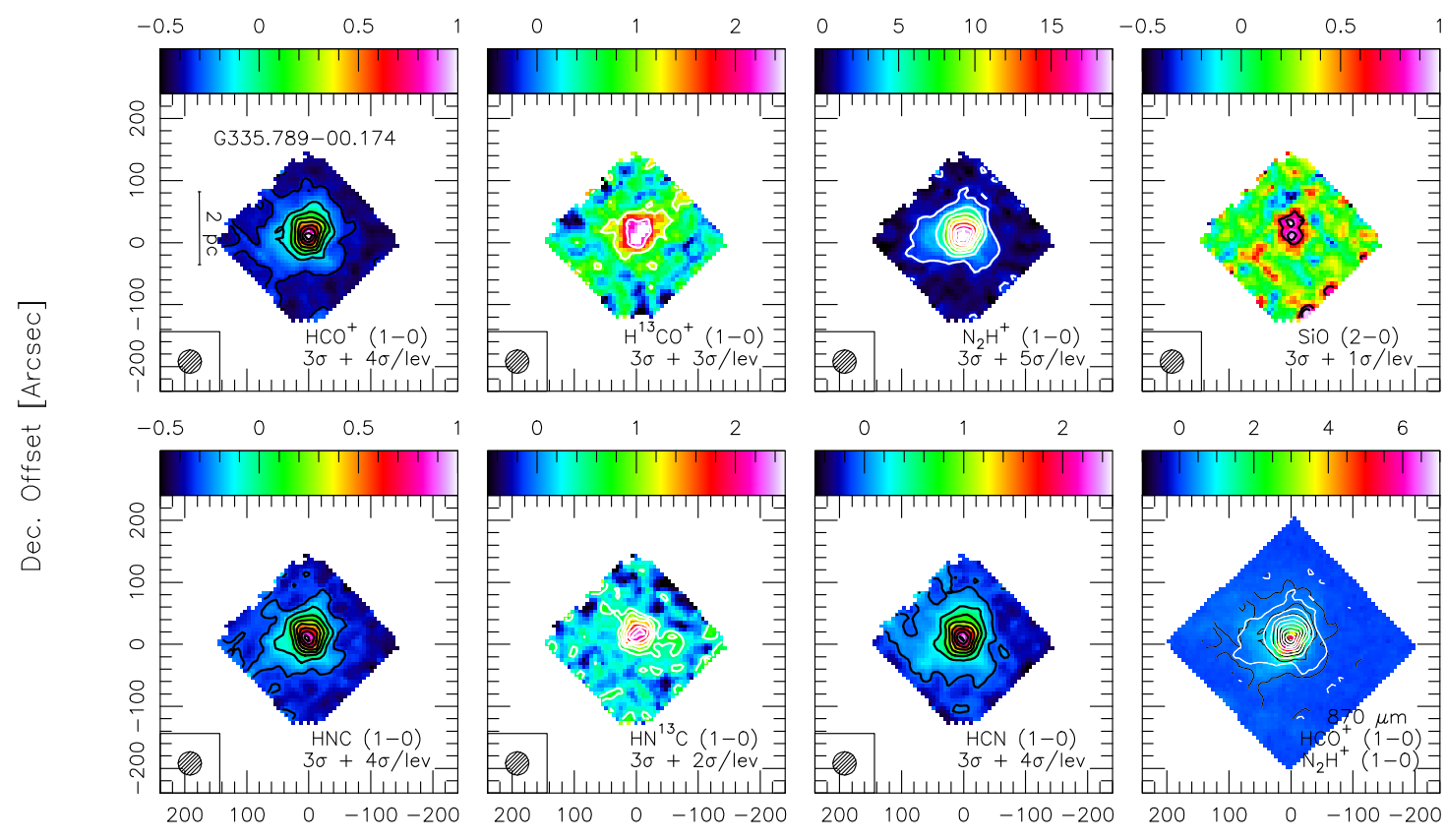

R.A. Offset [Arcsec]

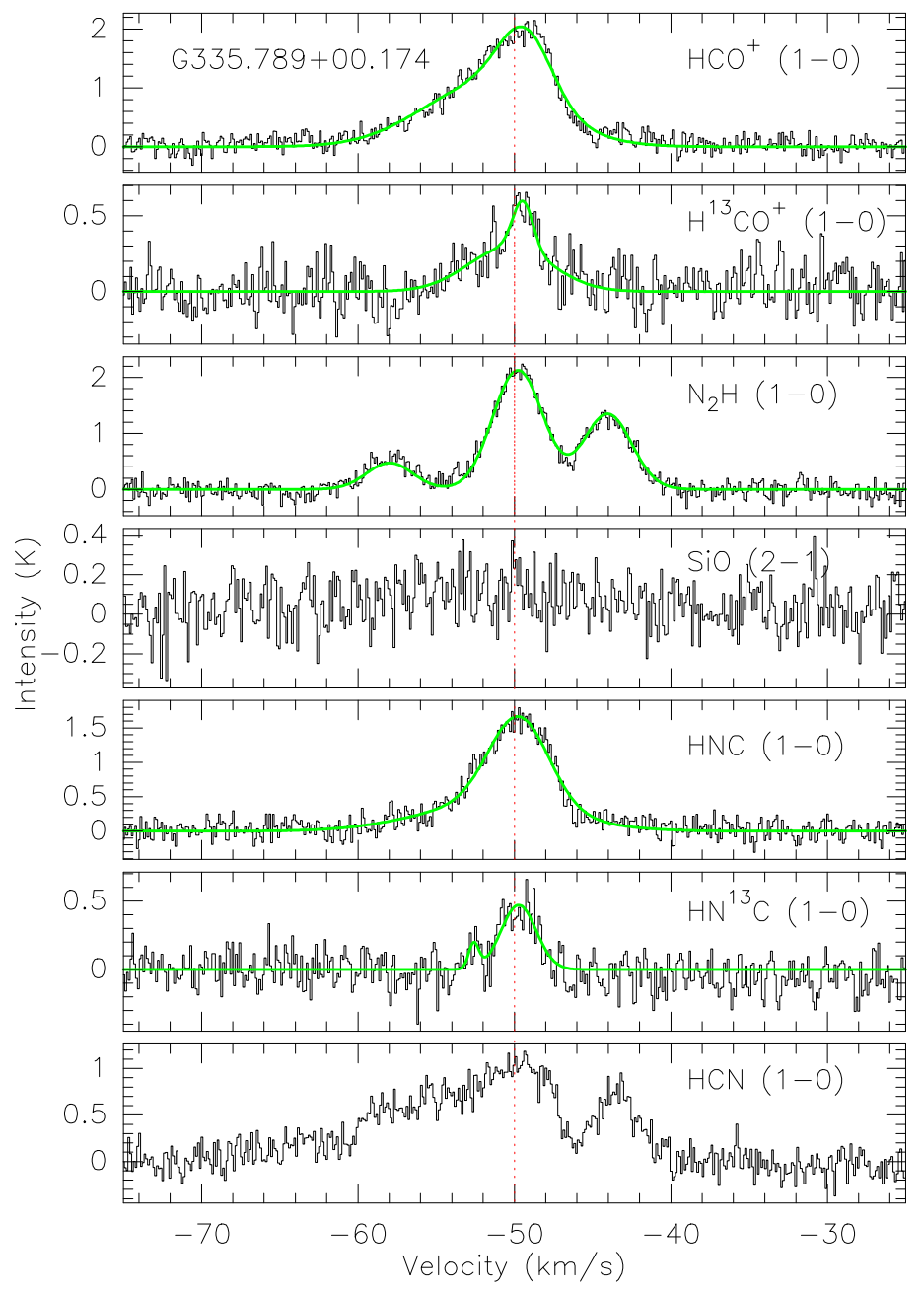

Fig. A.1. continued. 
G. Saral et al.: MALT90 molecular content on high-mass IR-dark clumps
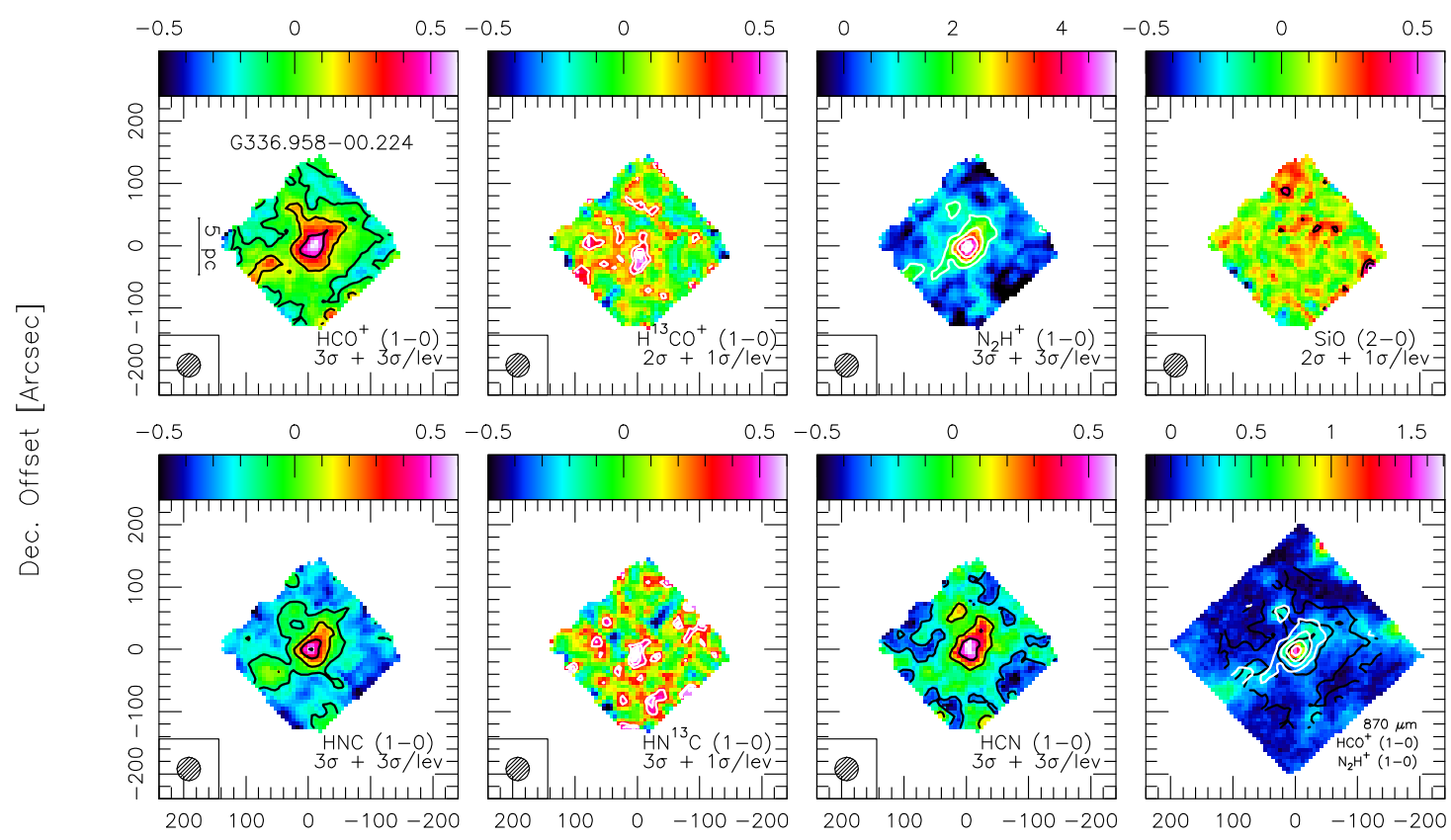

R.A. Offset [Arcsec]

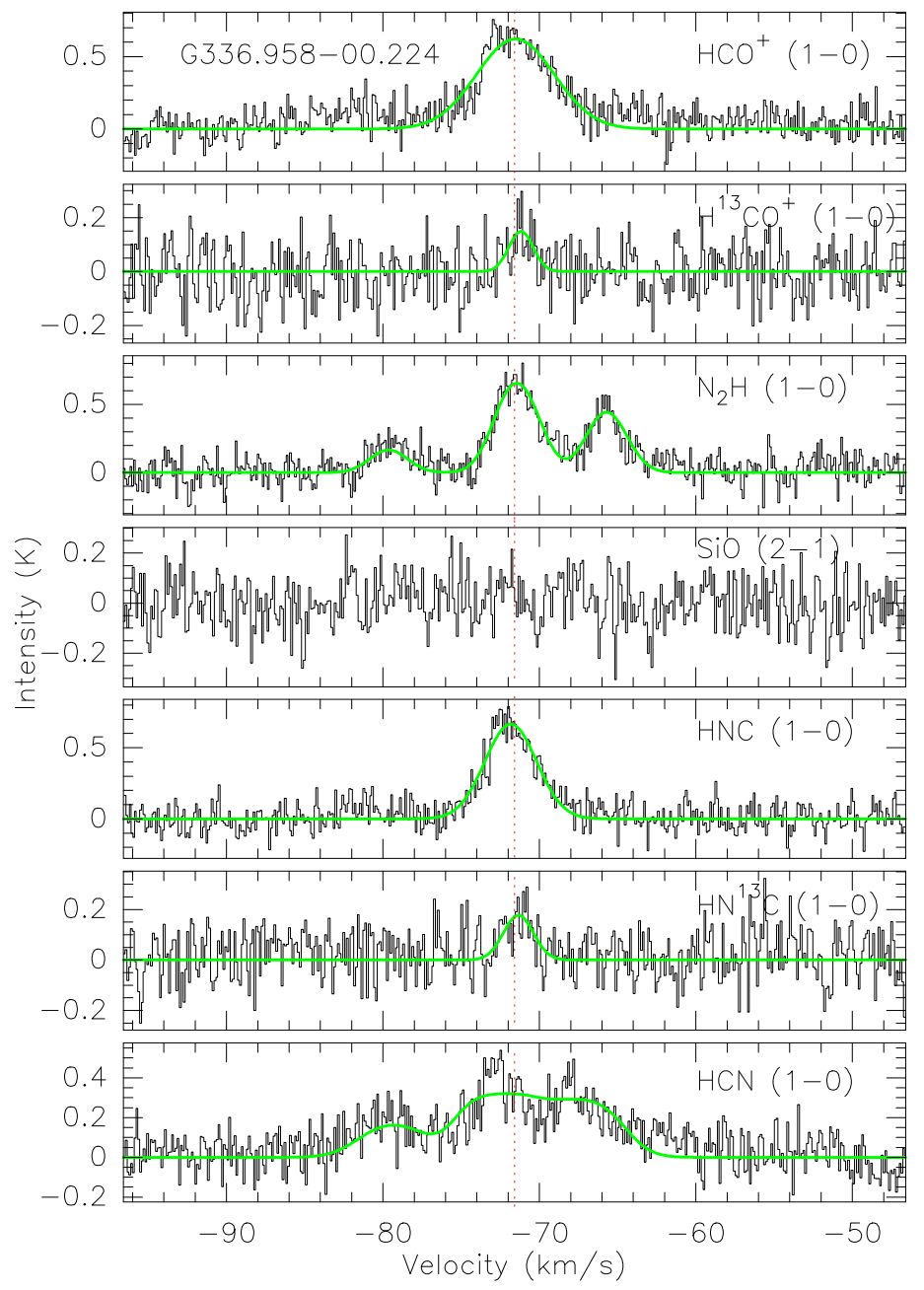

Fig. A.1. continued. 

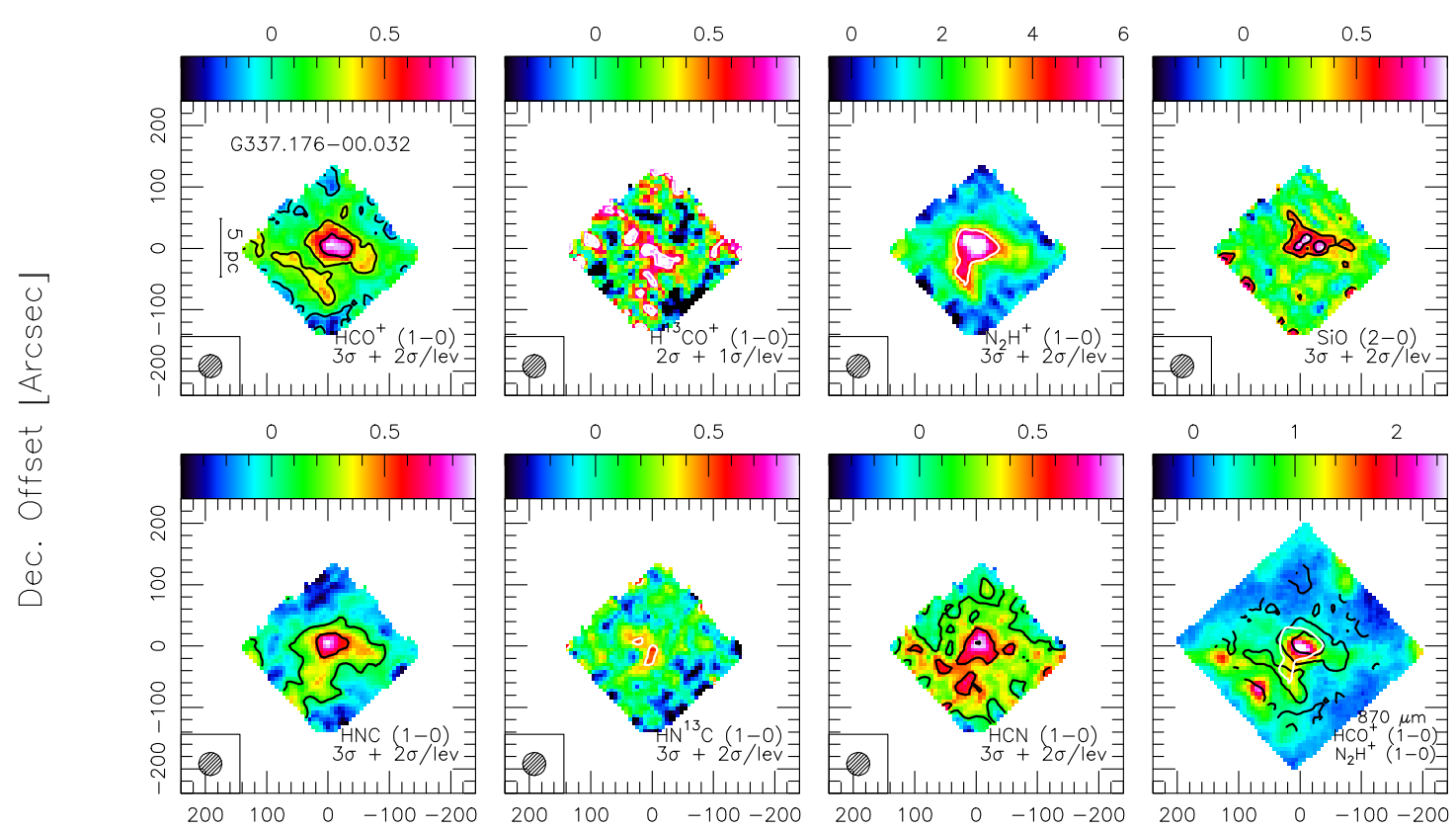

R.A. Offset [Arcsec]

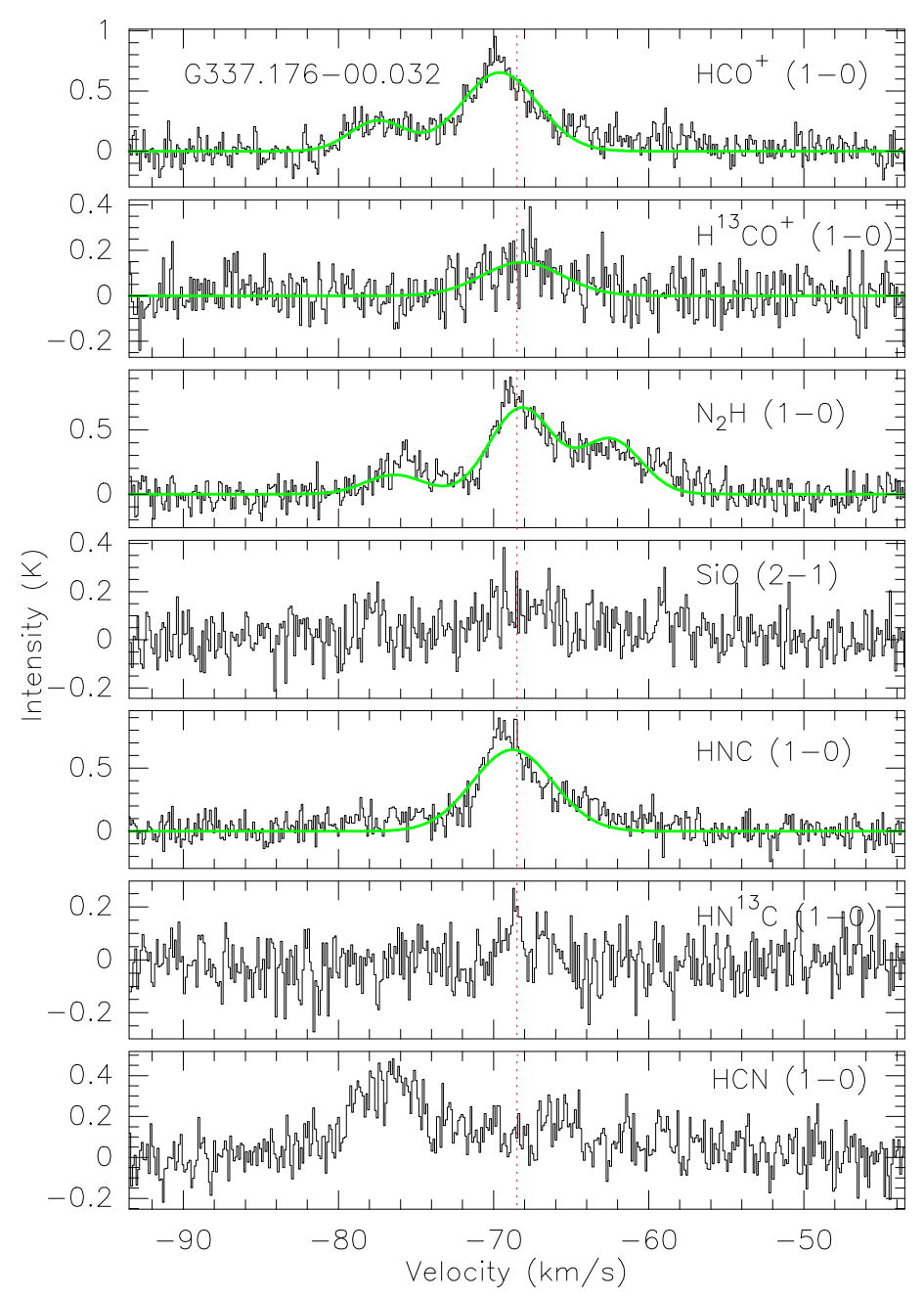

Fig. A.1. continued. 
G. Saral et al.: MALT90 molecular content on high-mass IR-dark clumps
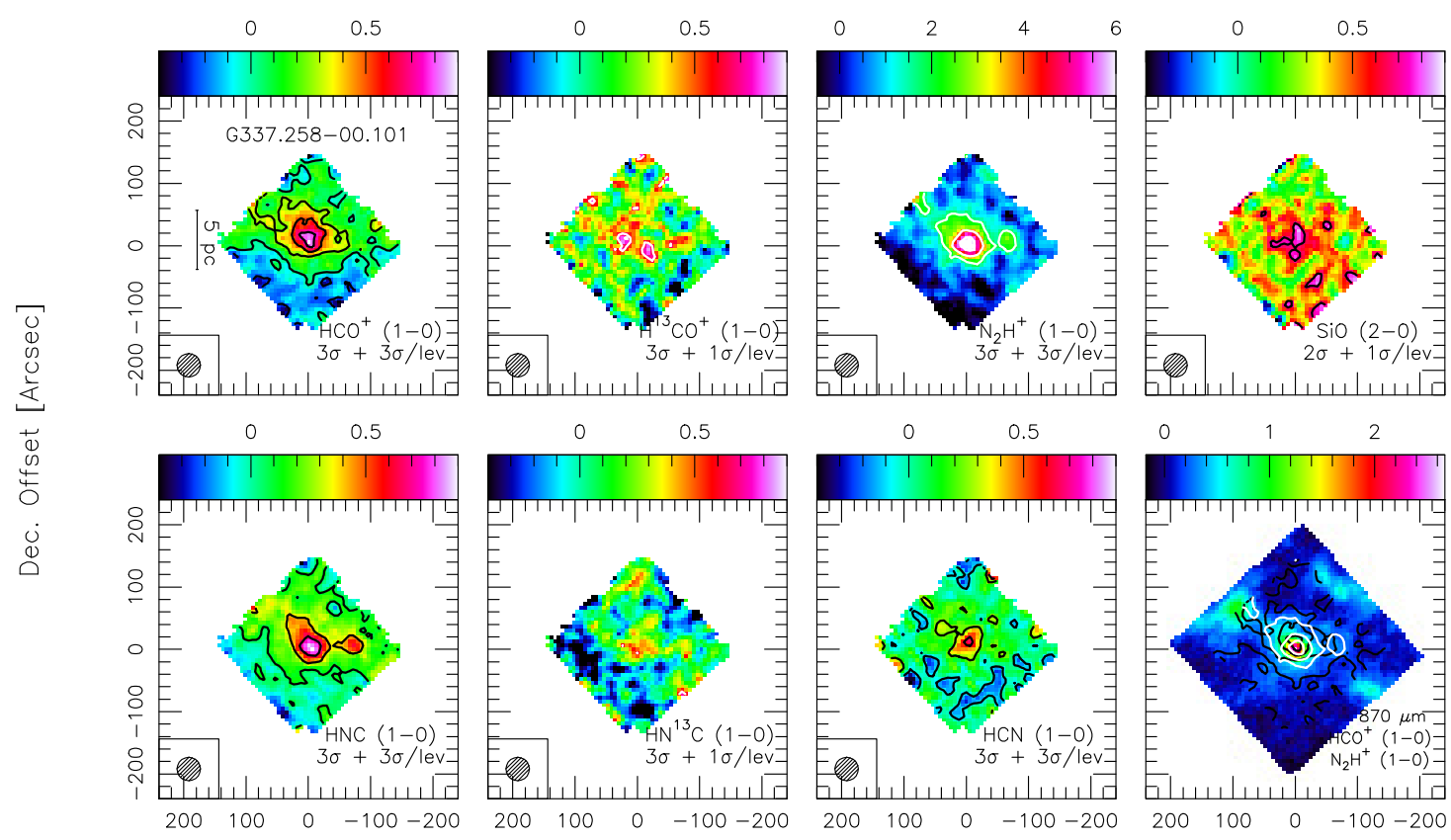

R.A. Offset [Arcsec]

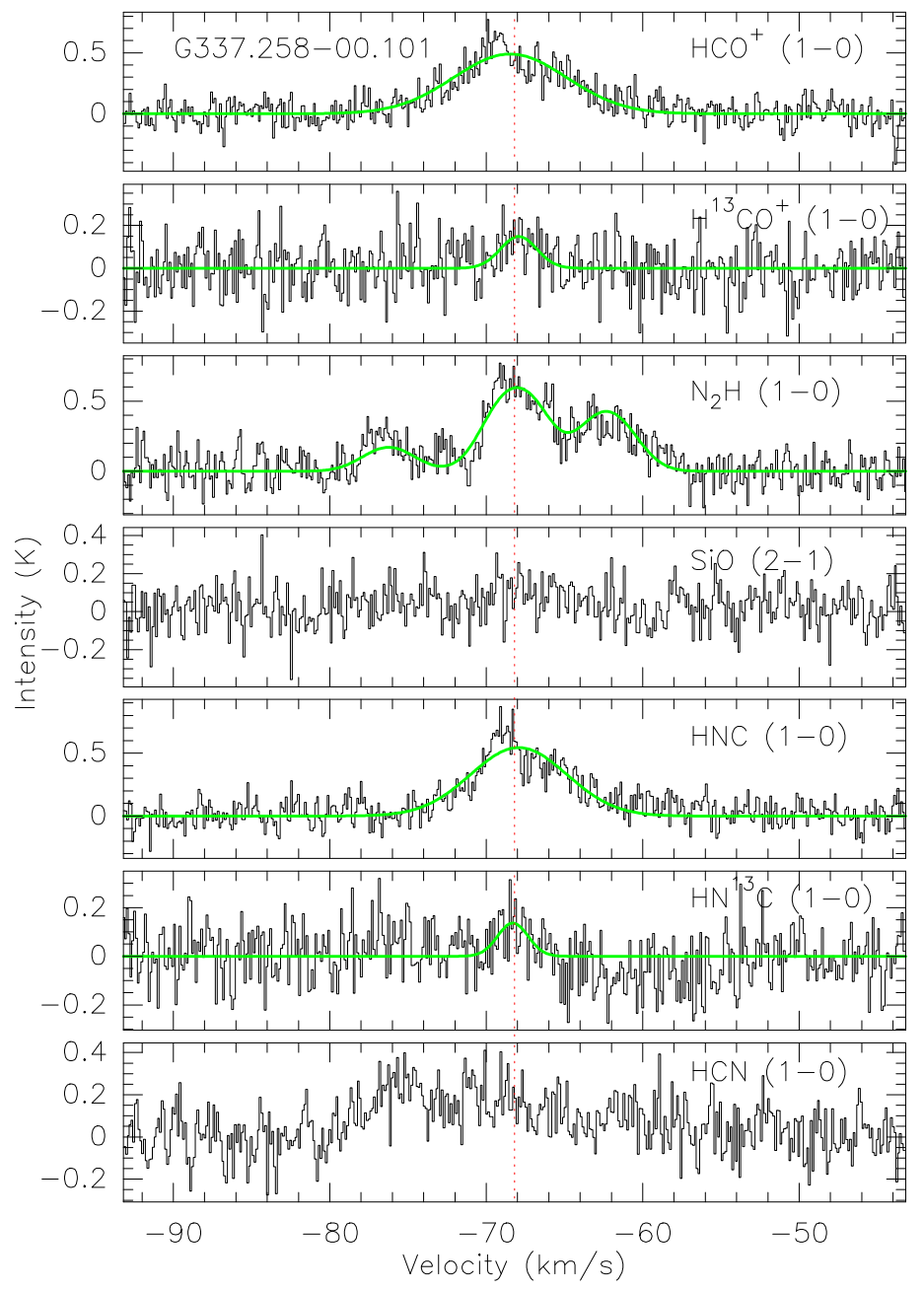

Fig. A.1. continued. 

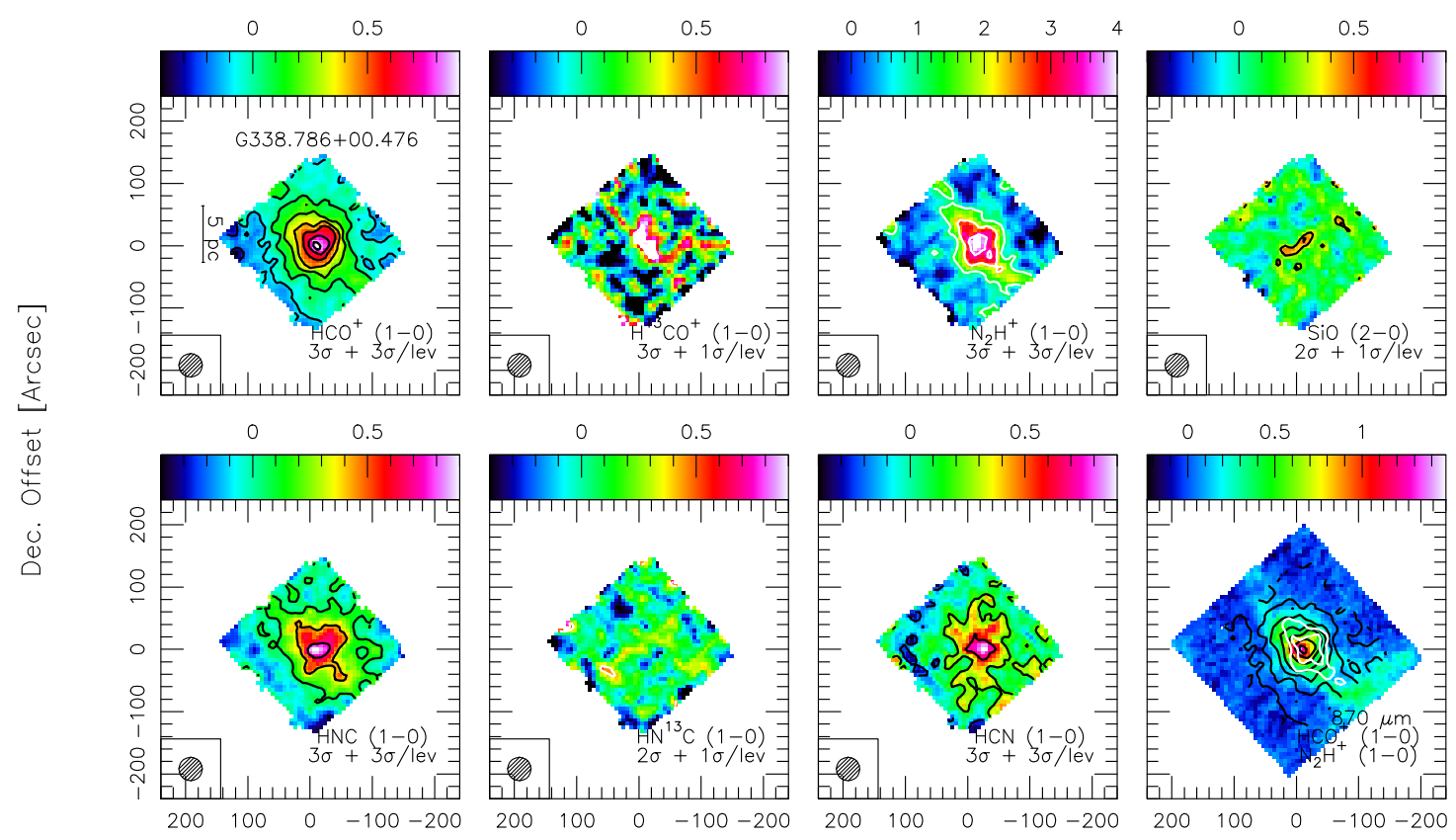

R.A. Offset [Arcsec]

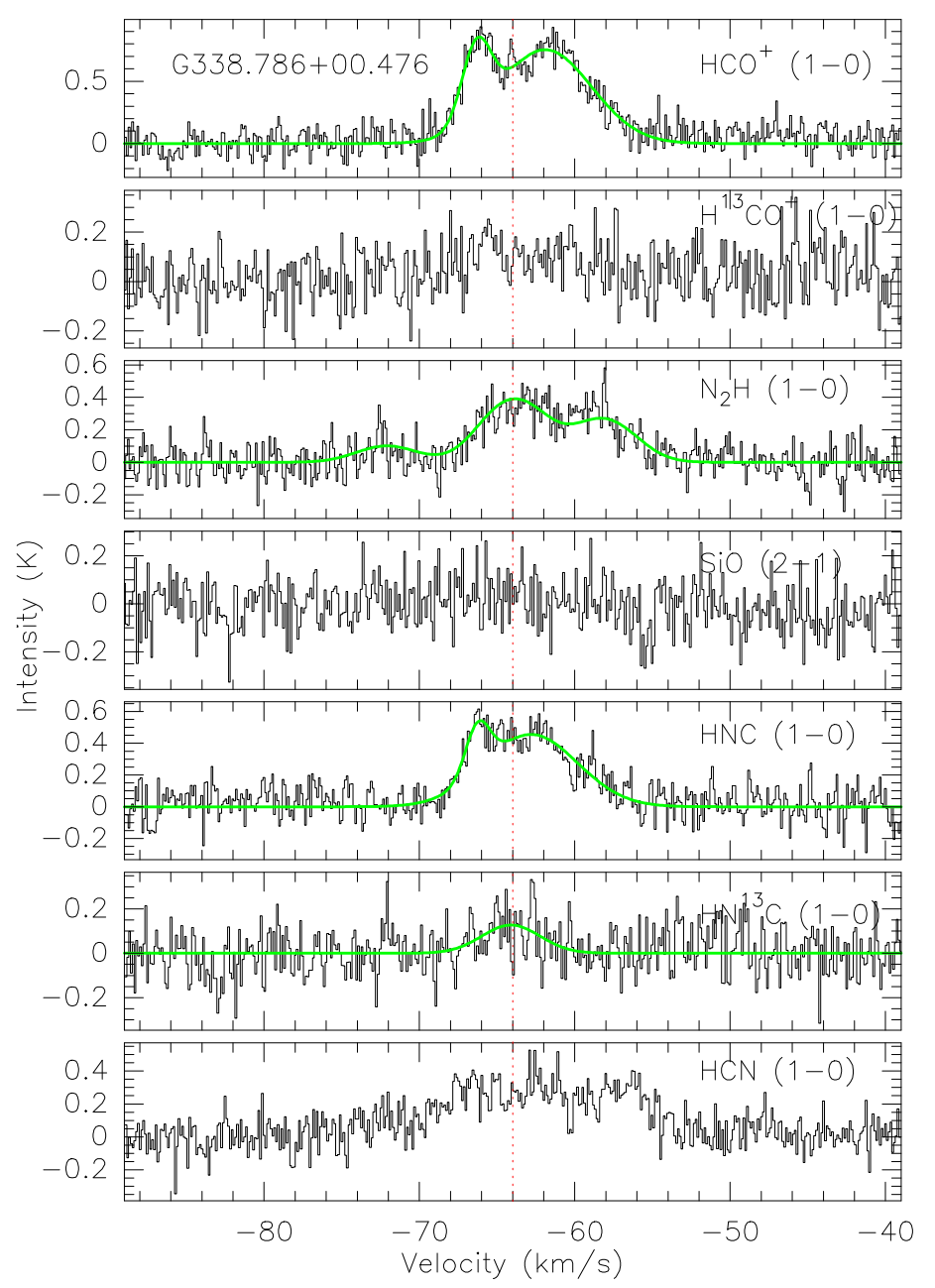

Fig. A.1. continued. 
G. Saral et al.: MALT90 molecular content on high-mass IR-dark clumps
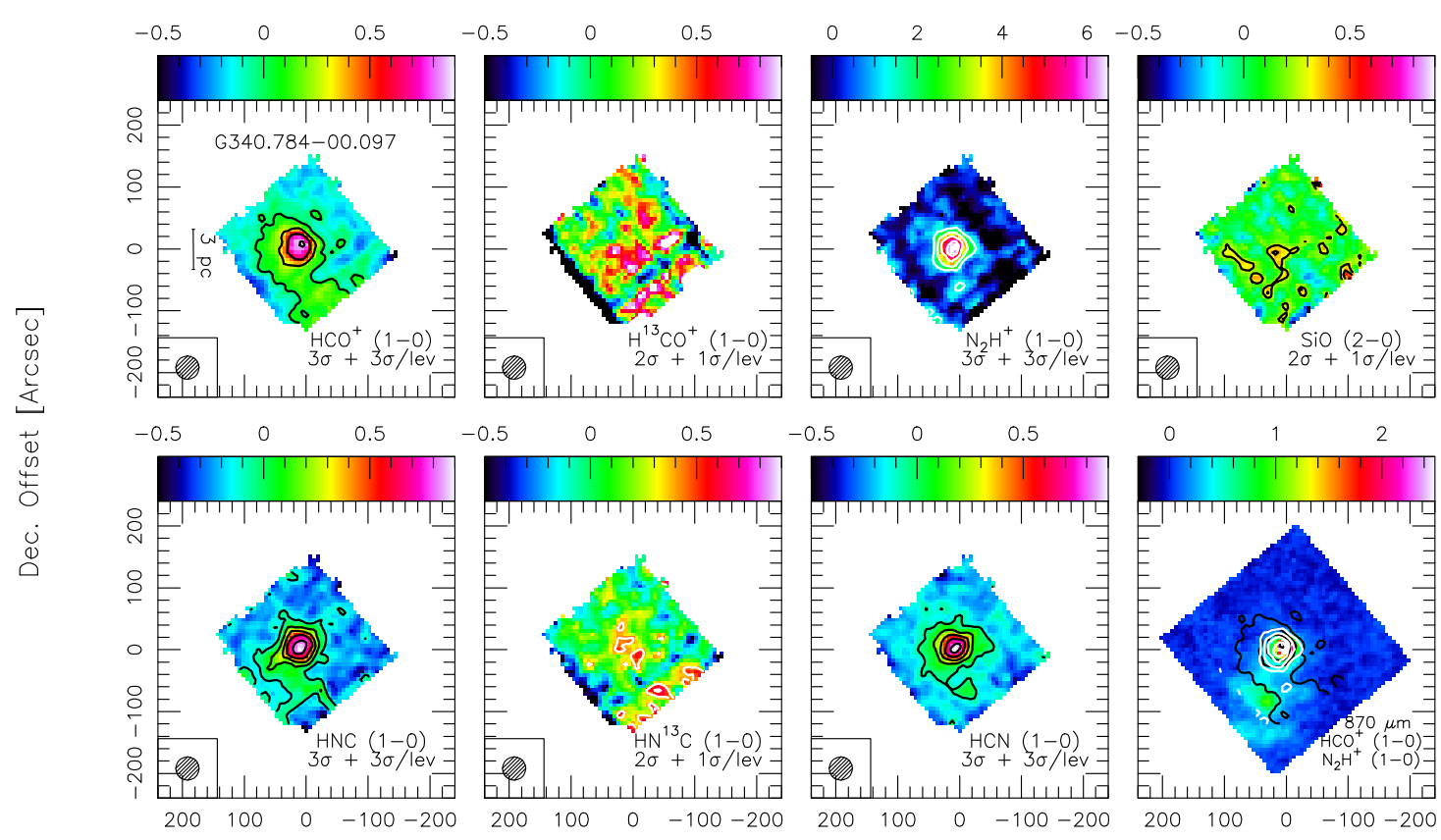

R.A. Offset [Arcsec]

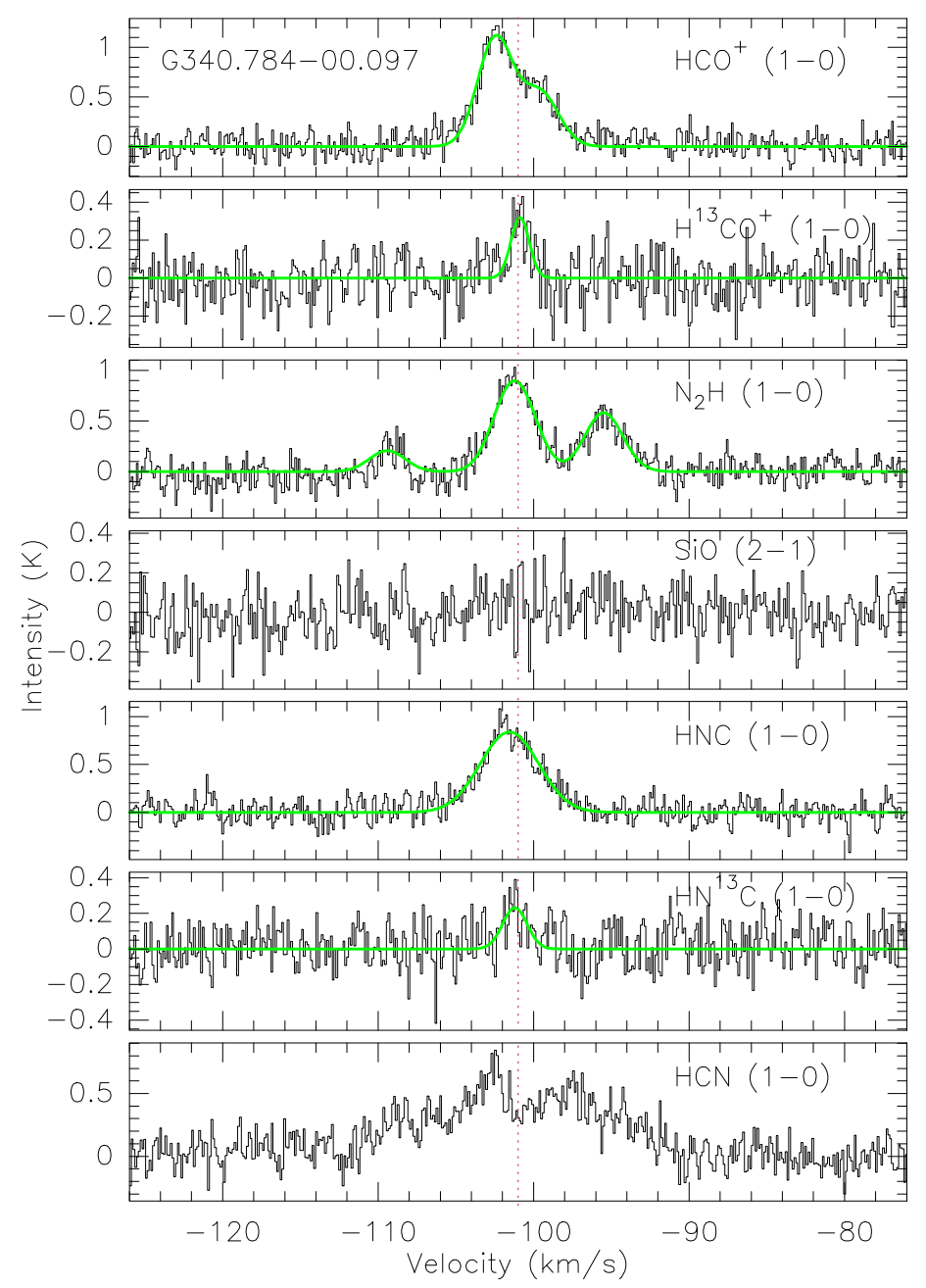

Fig. A.1. continued. 

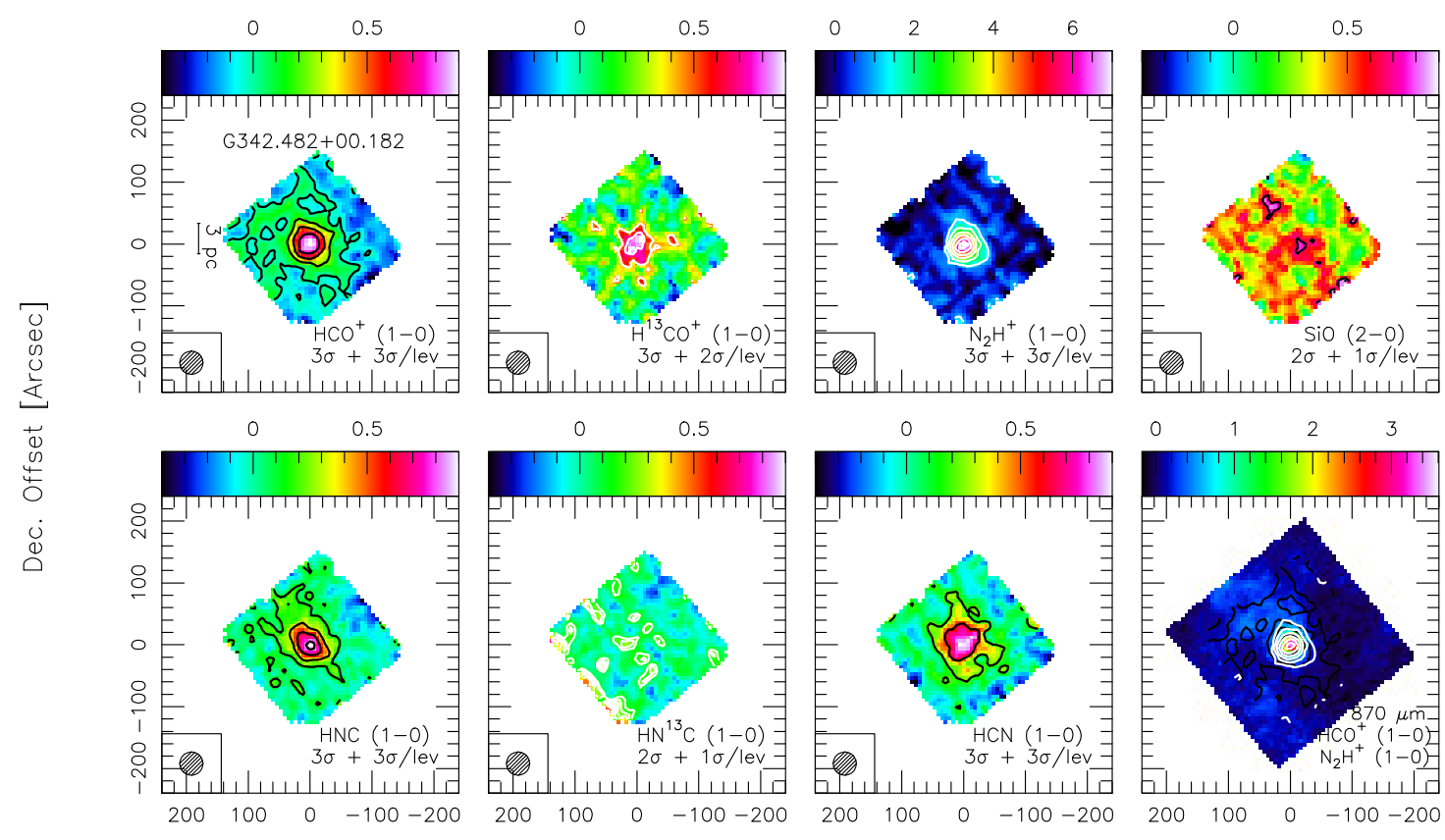

R.A. Offset [Arcsec]

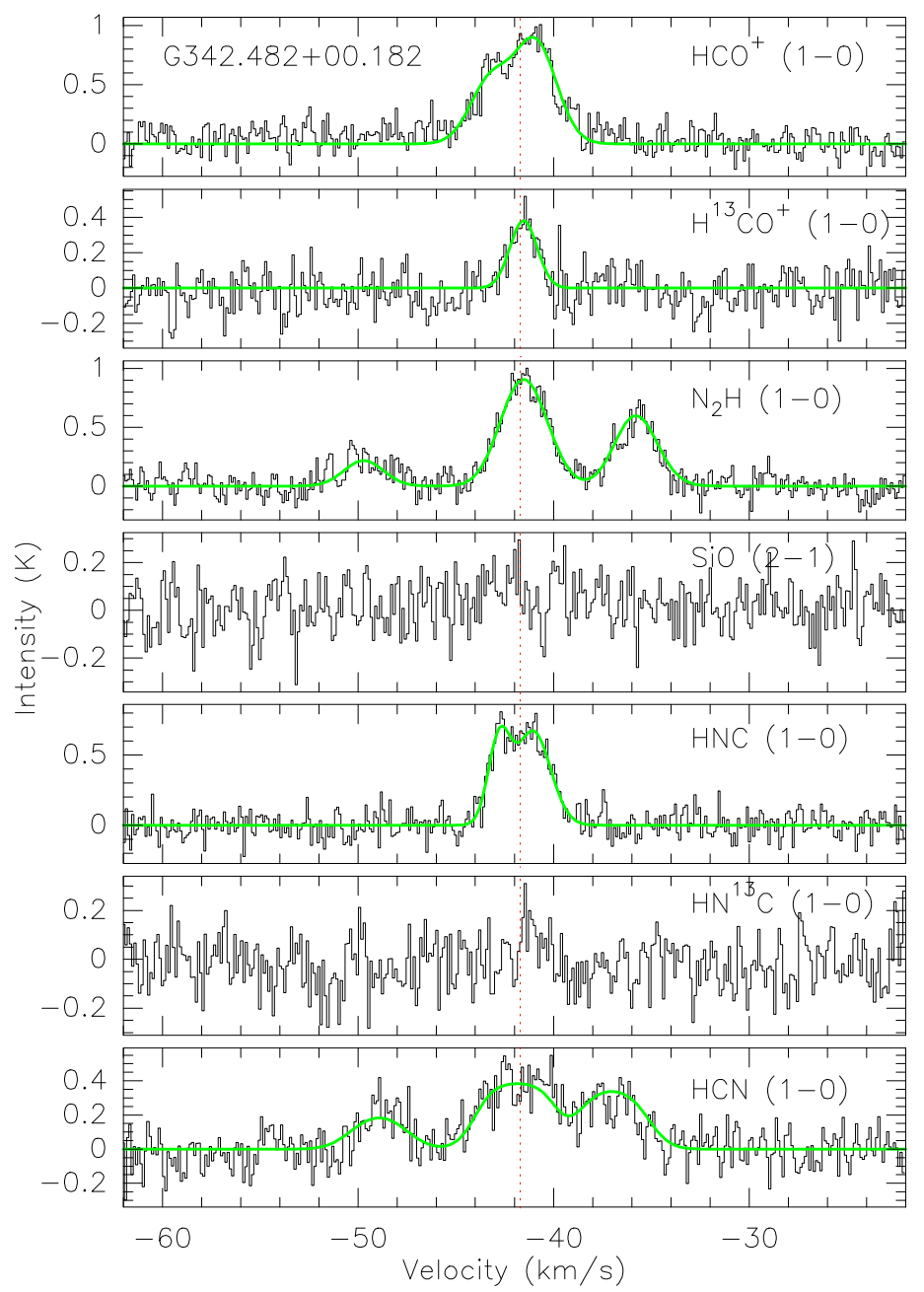

Fig. A.1. continued. 
G. Saral et al.: MALT90 molecular content on high-mass IR-dark clumps
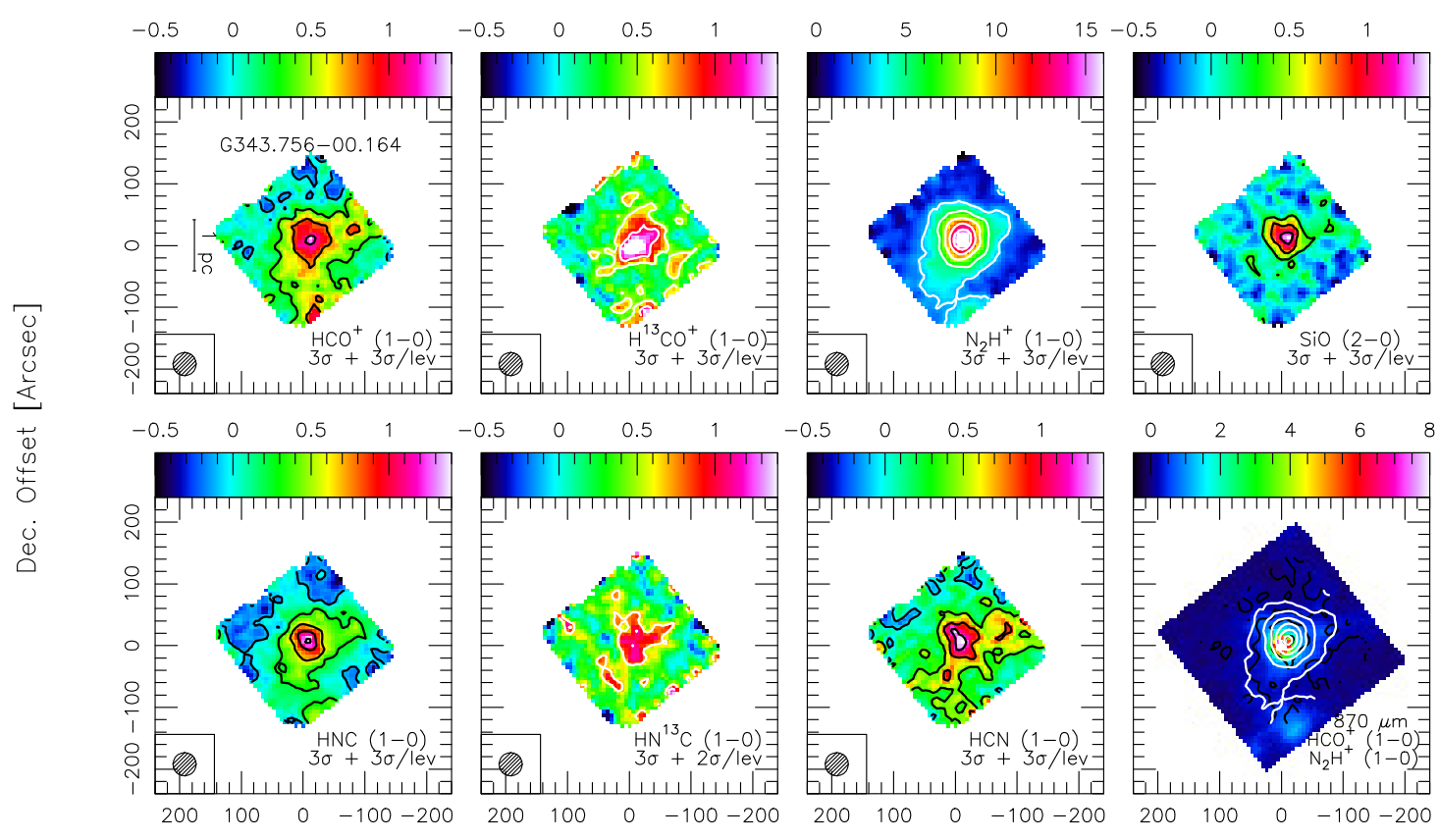

R.A. Offset [Arcsec]

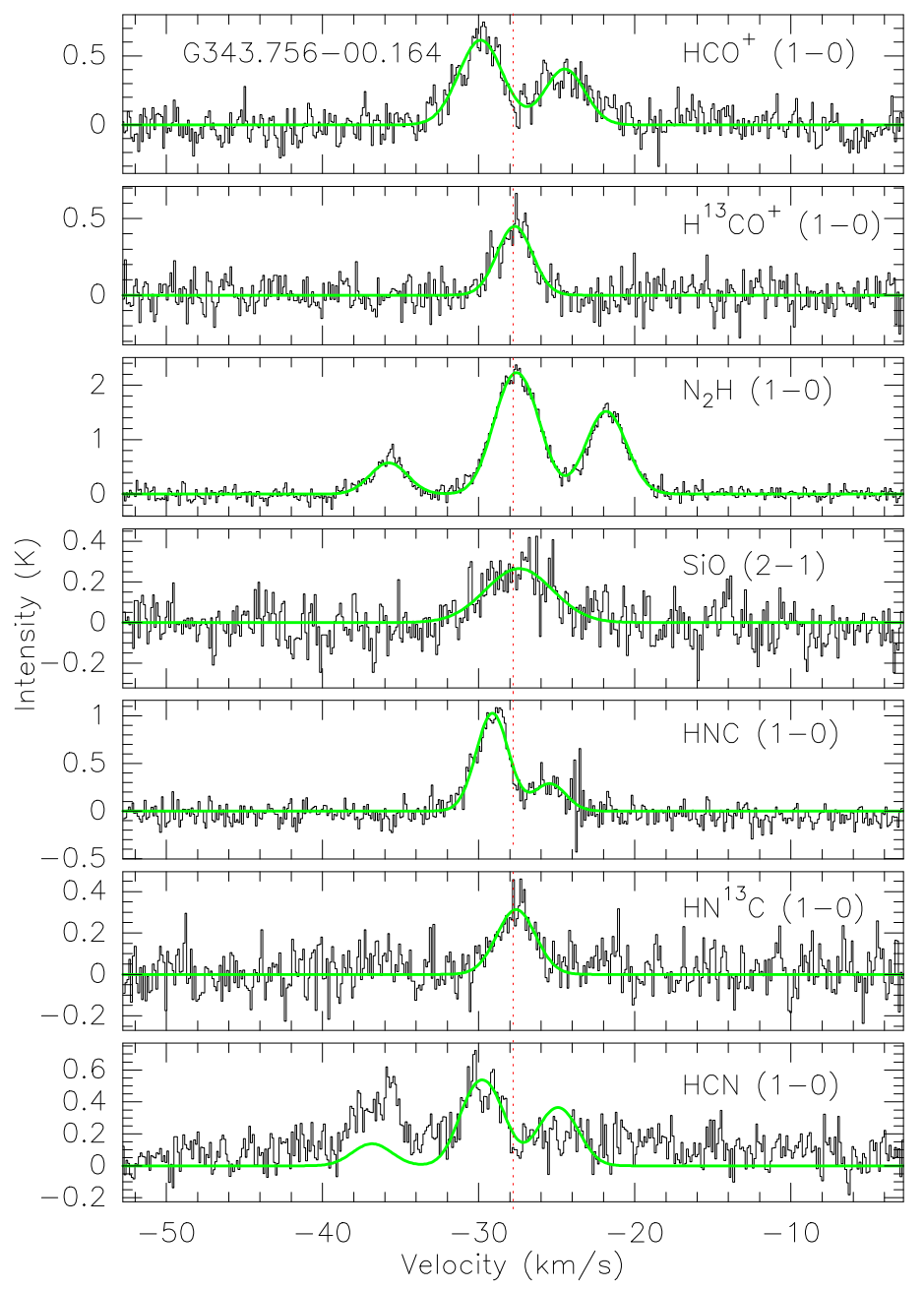

Fig. A.1. continued. 
A\&A 620, A158 (2018)
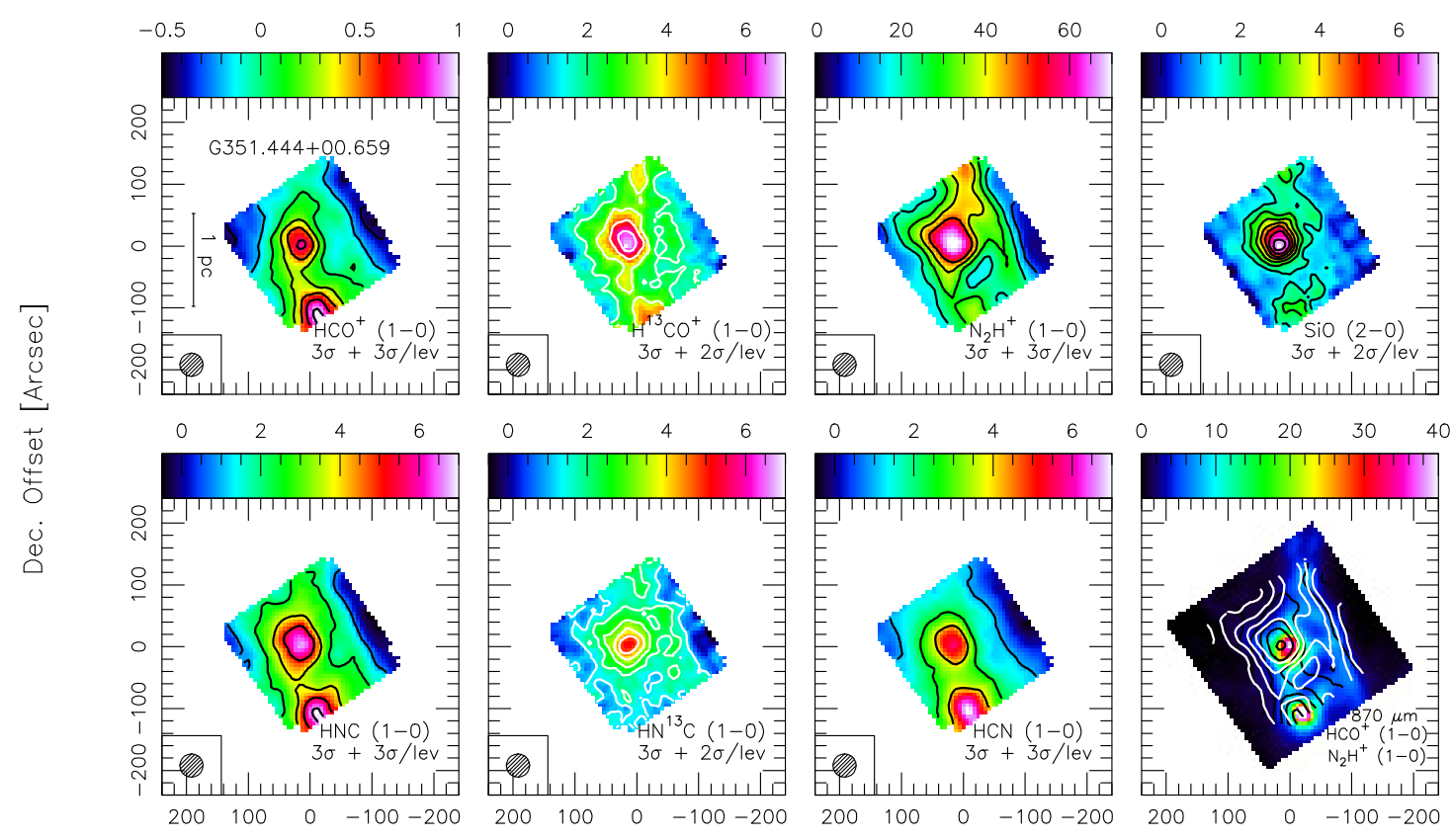

R.A. Offset [Arcsec]

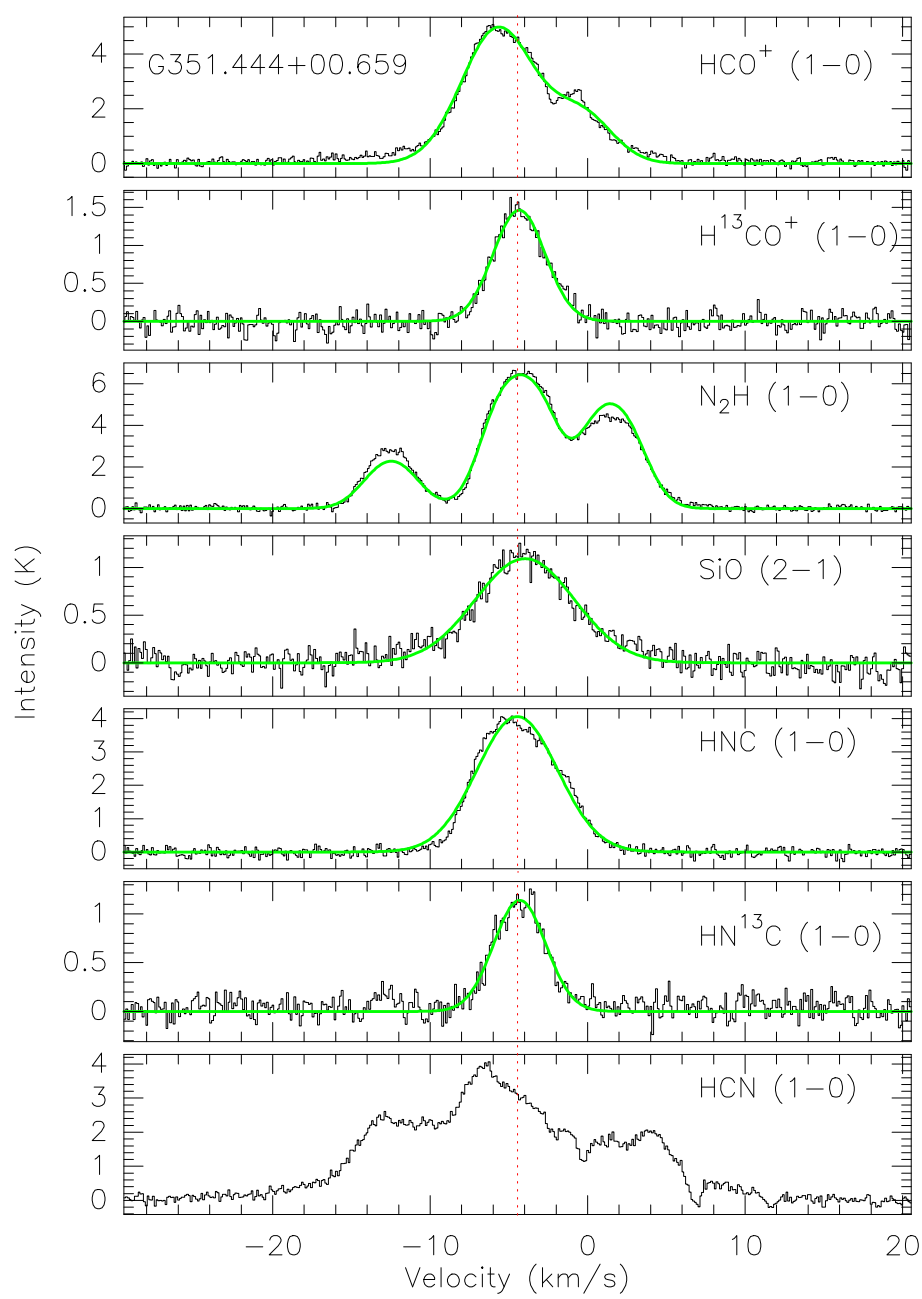

Fig. A.1. continued. 
G. Saral et al.: MALT90 molecular content on high-mass IR-dark clumps
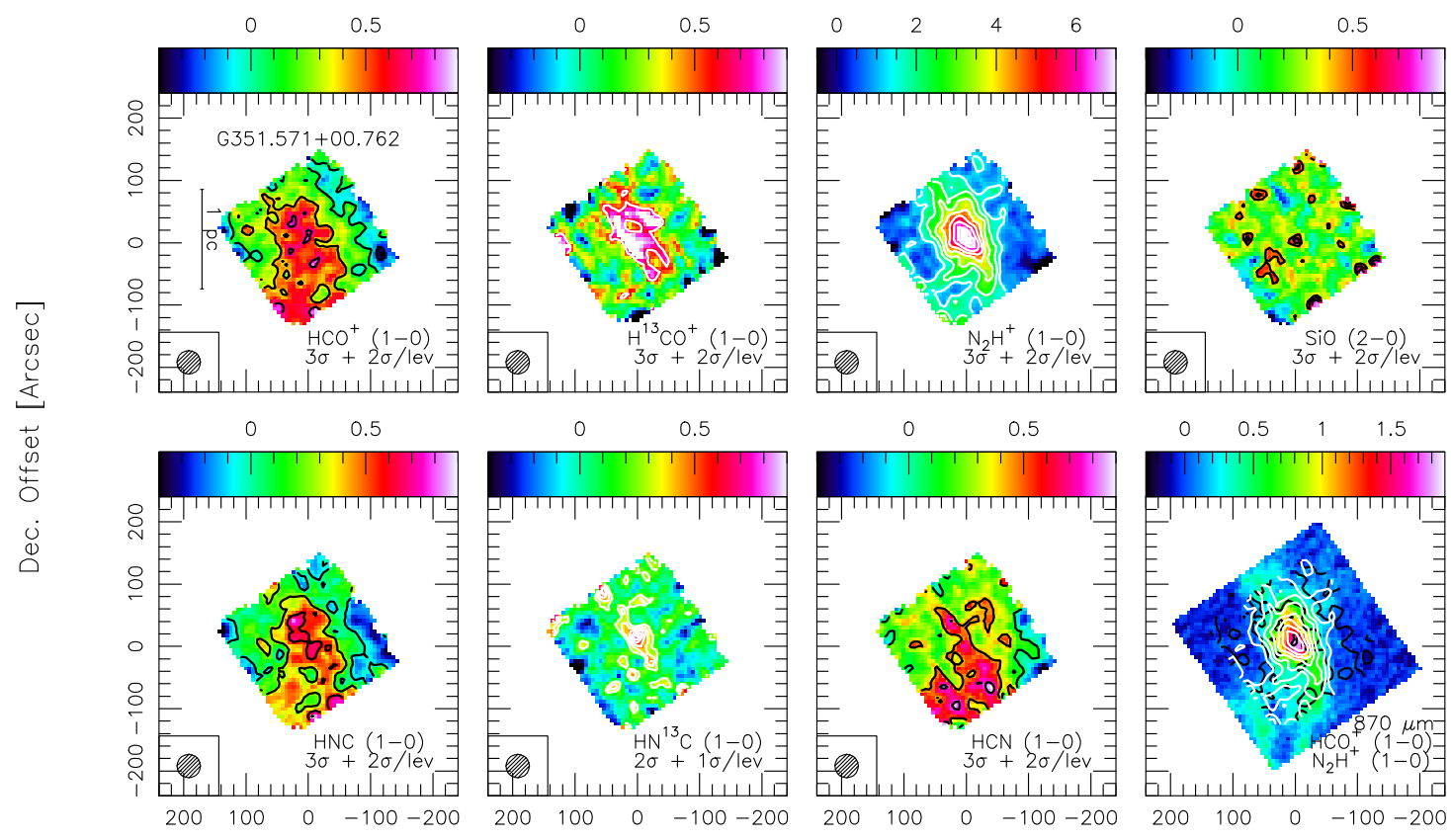

R.A. Offset [Arcsec]

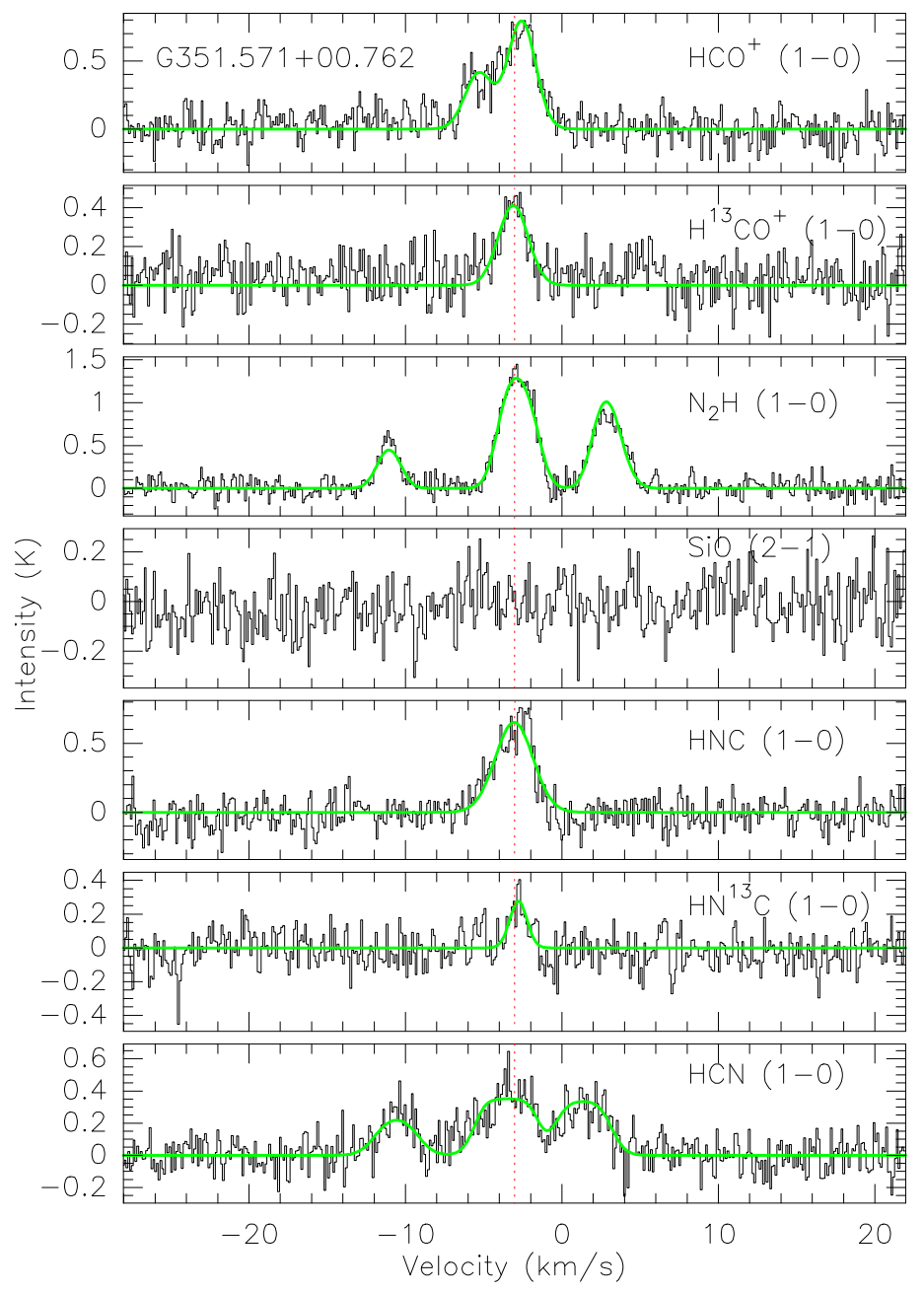

Fig. A.1. continued. 

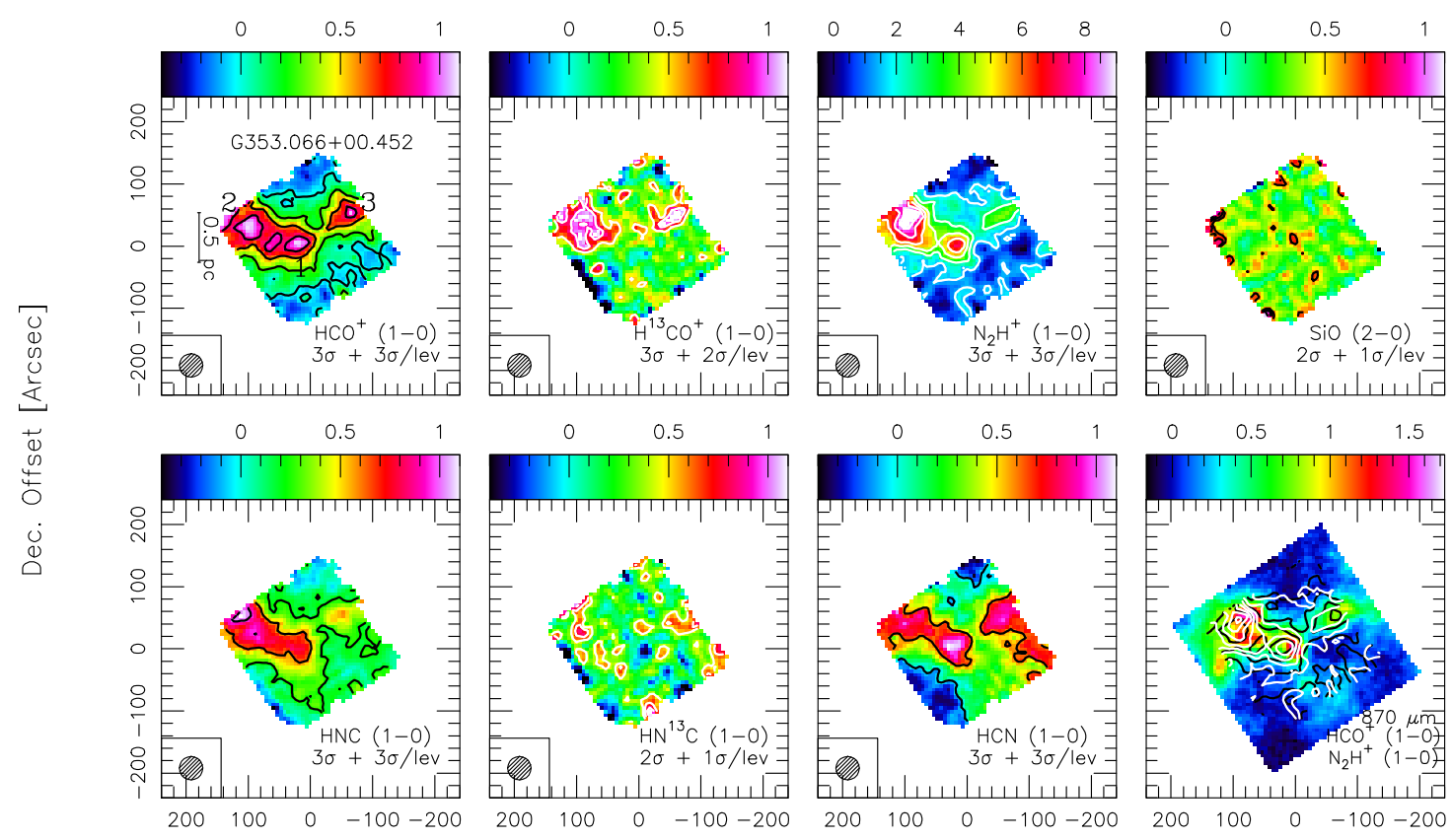

R.A. Offset [Arcsec]
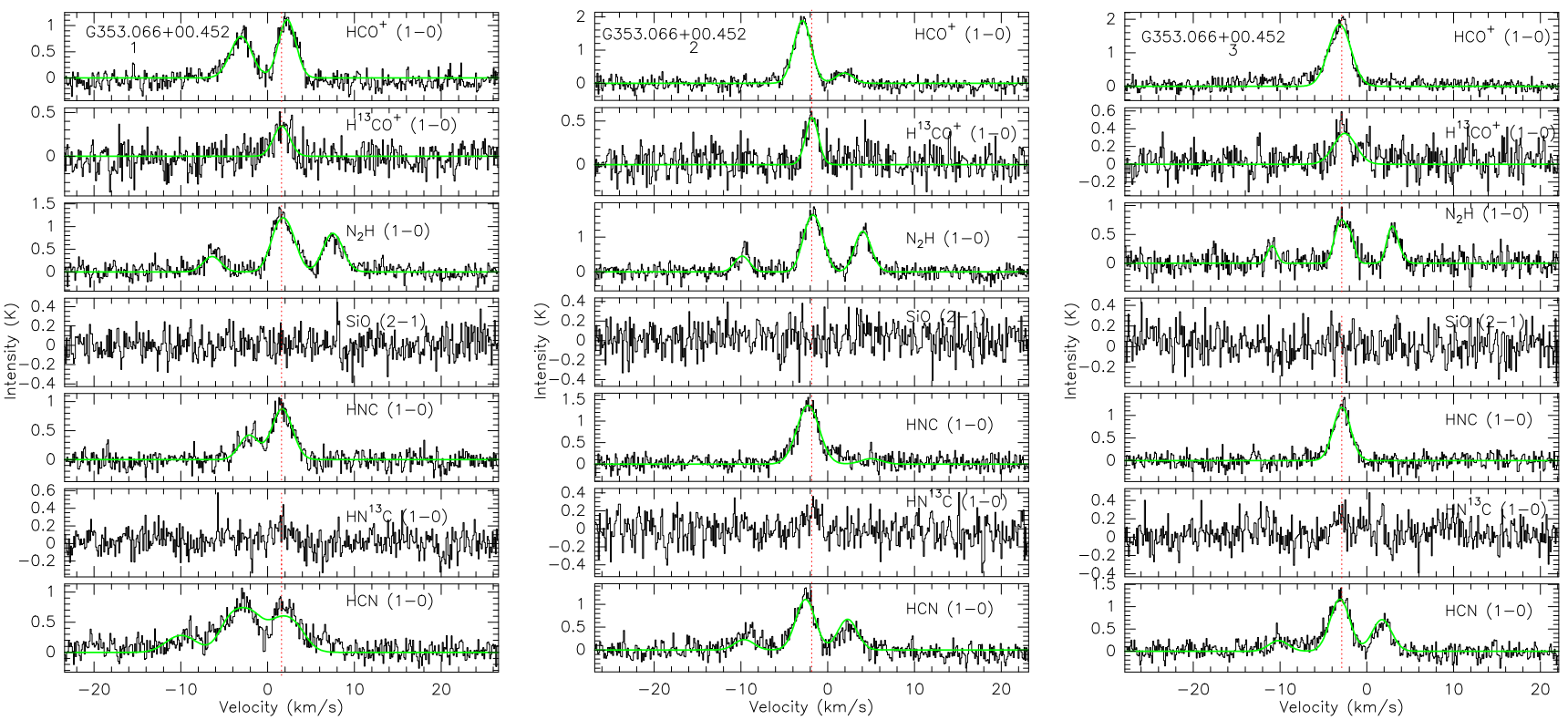

Fig. A.1. continued. 
G. Saral et al.: MALT90 molecular content on high-mass IR-dark clumps
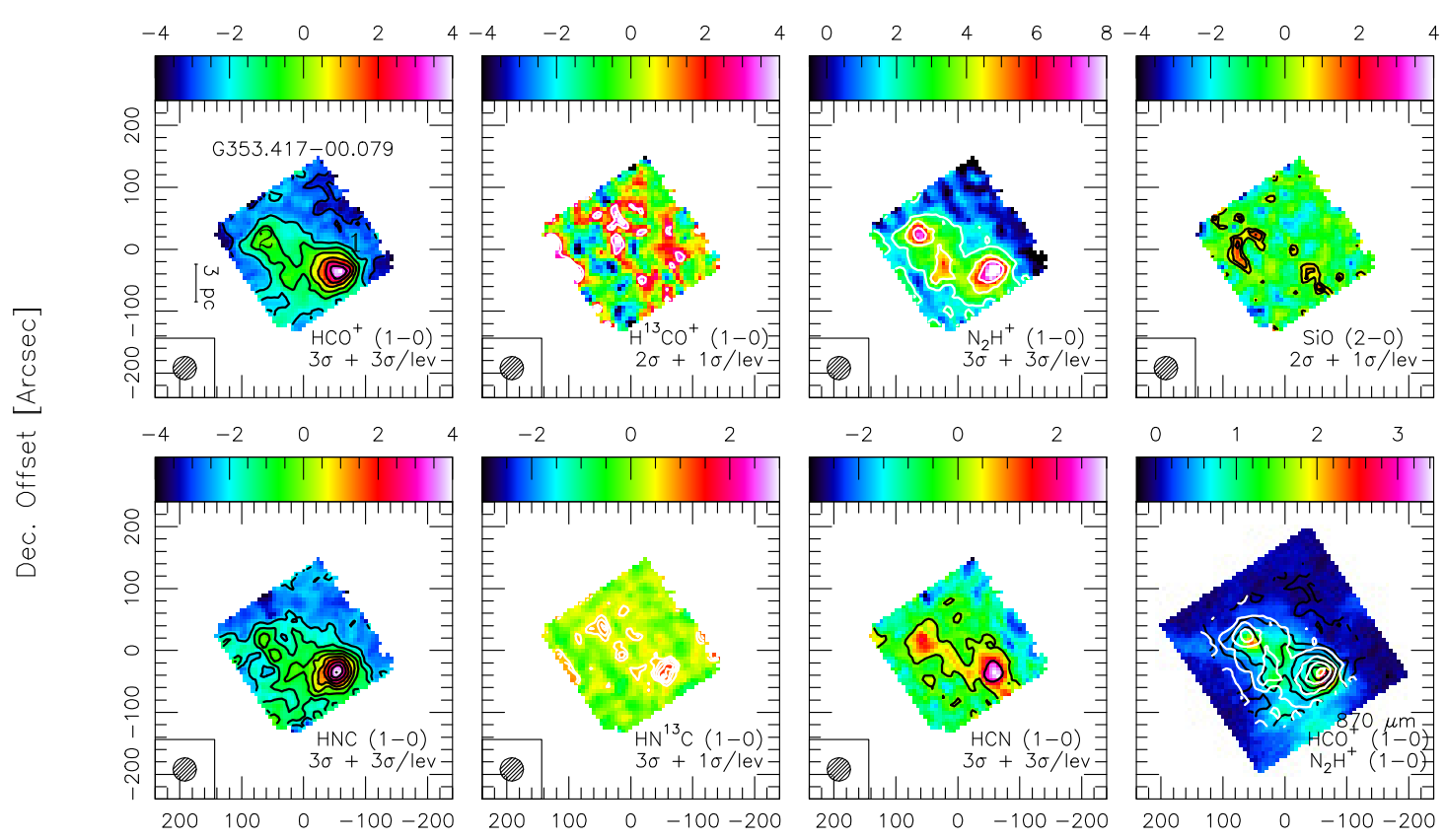

R.A. Offset [Arcsec]
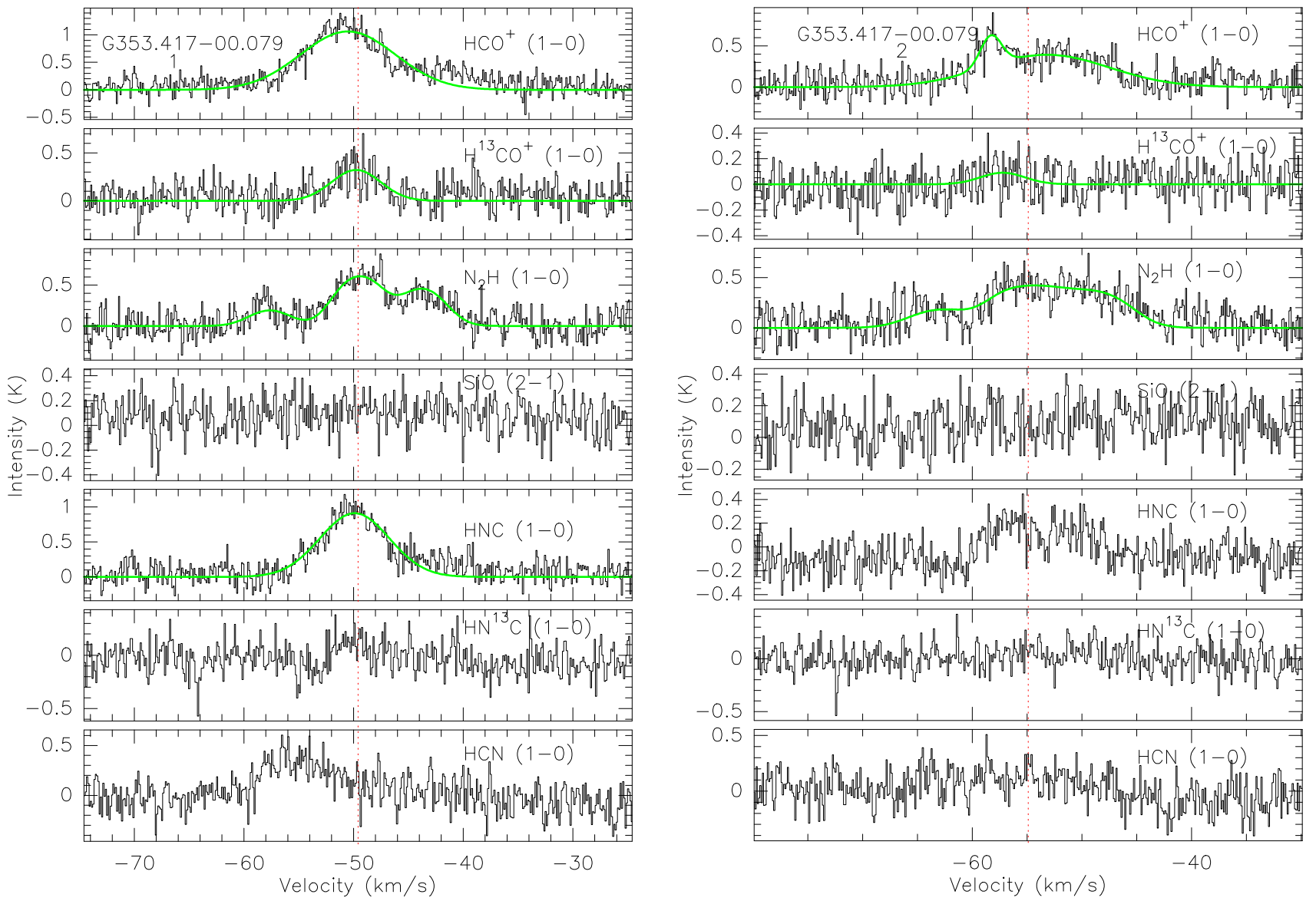

Fig. A.1. continued. 

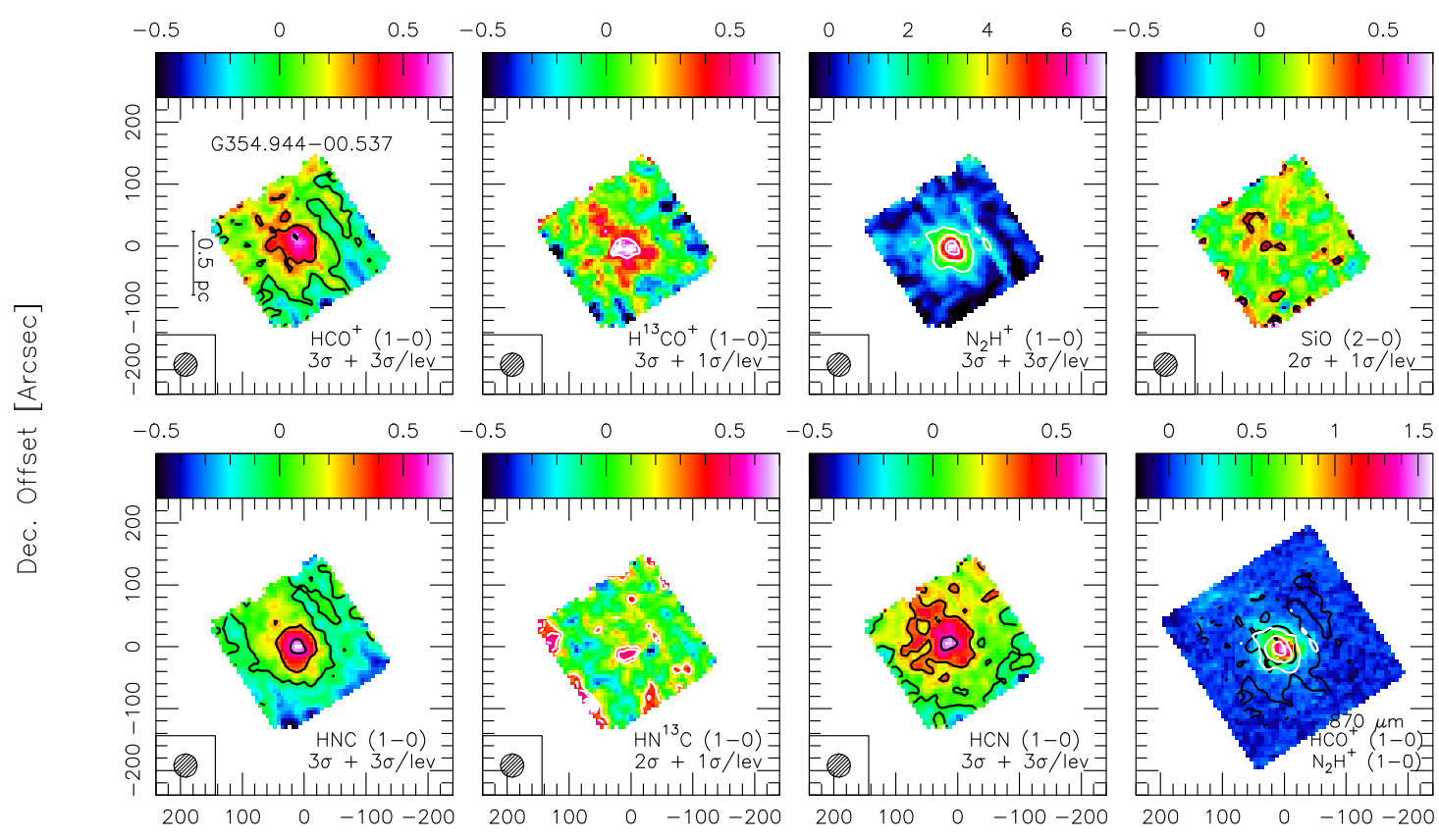

R.A. Offset [Arcsec]

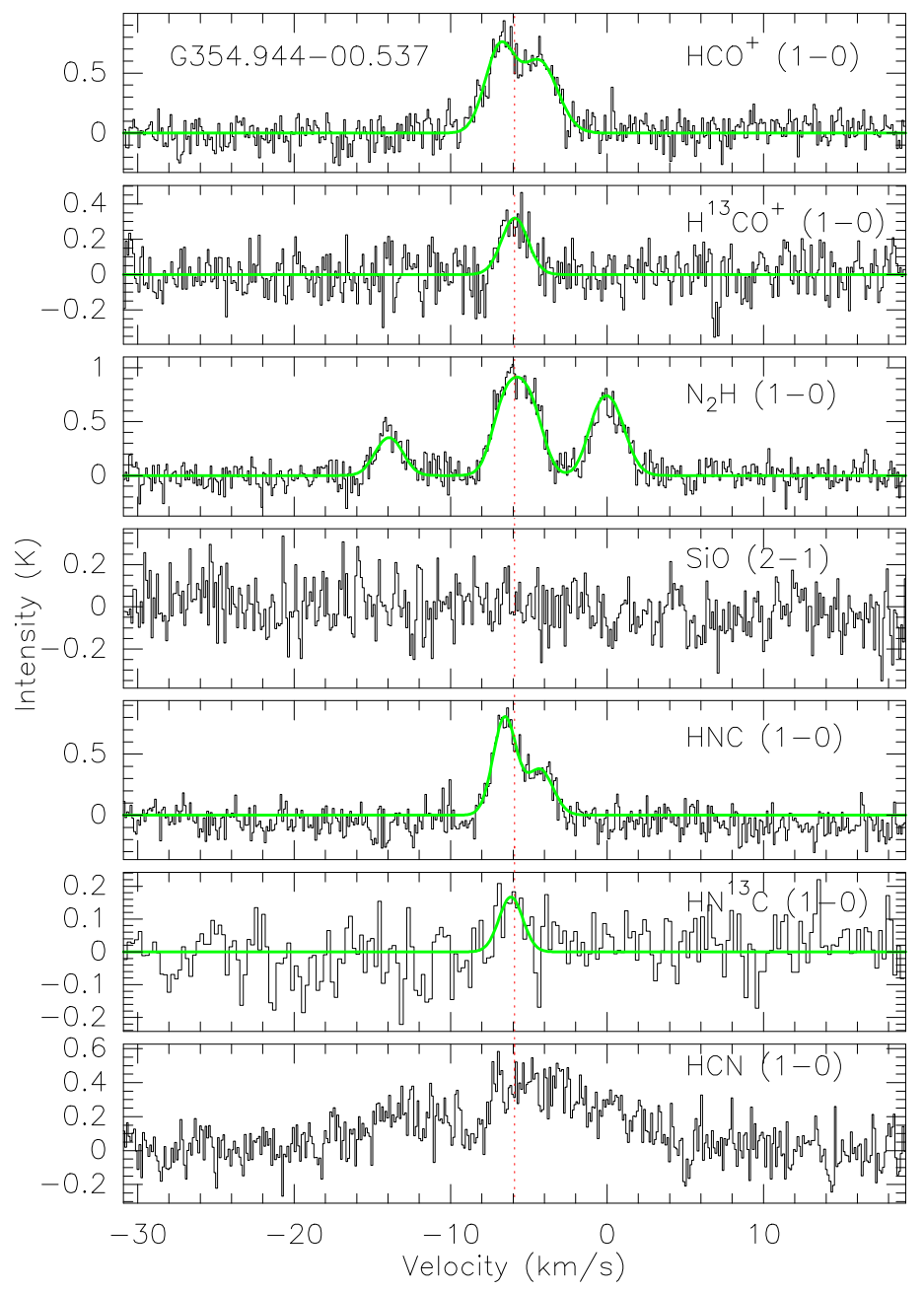

Fig. A.1. continued. 


\section{Appendix B: Additional tables}

Table B.1. Fitting results of the spectral lines.

\begin{tabular}{|c|c|c|c|c|c|c|}
\hline Source & Line properties & $\mathrm{HCO}^{+}(1-0)$ & $\mathrm{H}^{13} \mathrm{CO}^{+}(1-0)$ & $\begin{array}{r}\text { Lines } \\
\mathrm{SiO}(2-1) \\
\end{array}$ & $\mathrm{HNC}(1-0)$ & $\mathrm{HN}^{13} \mathrm{C}(1-0)$ \\
\hline \multirow{4}{*}{ G008.684-00.367 } & $\mathrm{W}\left(\mathrm{K} \mathrm{km} \mathrm{s}^{-1}\right)^{a}$ & $6.58(0.18)^{b}$ & $2.41(0.11)$ & $1.48(0.15)$ & $2.35(0.38)^{b}$ & $1.98(0.12)$ \\
\hline & $V_{\mathrm{LSR}}\left(\mathrm{km} \mathrm{s}^{-1}\right)$ & $34.04(0.04)^{b}$ & $36.94(0.10)$ & $37.71(0.42)$ & $34.22(0.06)^{b}$ & $37.40(0.17)$ \\
\hline & $\Delta V\left(\mathrm{~km} \mathrm{~s}^{-1}\right)$ & $3.70(0.10)^{b}$ & $4.46(0.23)$ & $8.09(0.94)$ & $2.72(0.24)^{b}$ & $5.33(0.35)$ \\
\hline & $T_{\mathrm{mb}}(\mathrm{K})$ & $1.67^{b}$ & 0.51 & 0.17 & $0.81^{b}$ & 0.35 \\
\hline \multirow{4}{*}{ G008.706-00.414 } & $\mathrm{W}\left(\mathrm{K} \mathrm{km} \mathrm{s}^{-1}\right)$ & $7.42(0.11)$ & $0.96(0.08)$ & $0.82(0.11)$ & $5.19(0.10)$ & $0.58(0.07)$ \\
\hline & $V_{\mathrm{LSR}}\left(\mathrm{km} \mathrm{s}^{-1}\right)$ & $38.56(0.04)$ & $39.09(0.12)$ & $39.35(0.32)$ & $38.65(0.04)$ & $39.38(0.11)$ \\
\hline & $\Delta V\left(\mathrm{~km} \mathrm{~s}^{-1}\right)$ & $5.30(0.09)$ & $2.82(0.27)$ & $4.38(0.77)$ & $4.20(0.10)$ & $1.81(0.22)$ \\
\hline & $T_{\mathrm{mb}}(\mathrm{K})$ & 1.32 & 0.32 & 0.18 & 1.16 & 0.30 \\
\hline \multirow{4}{*}{ G010.444-00.017 } & $\mathrm{W}\left(\mathrm{K} \mathrm{km} \mathrm{s}^{-1}\right)$ & $3.13(0.16)^{c}$ & - & - & $0.94(0.12)^{c}$ & - \\
\hline & $V_{\mathrm{LSR}}\left(\mathrm{km} \mathrm{s}^{-1}\right)$ & $74.00(0.14)^{c}$ & - & - & $66.17(0.22)^{c}$ & - \\
\hline & $\Delta V\left(\mathrm{~km} \mathrm{~s}^{-1}\right)$ & $6.69(0.47)^{c}$ & - & - & $4.11(0.61)^{c}$ & - \\
\hline & $T_{\mathrm{mb}}(\mathrm{K})$ & $0.44^{c}$ & - & - & $0.21^{c}$ & - \\
\hline \multirow{4}{*}{ G013.178+00.059 } & $\mathrm{W}\left(\mathrm{K} \mathrm{km} \mathrm{s}^{-1}\right)$ & $5.60(0.14)$ & $0.83(0.12)$ & $1.12(0.16)$ & $6.31(0.11)$ & $1.12(0.09)$ \\
\hline & $V_{\mathrm{LSR}}\left(\mathrm{km} \mathrm{s}^{-1}\right)$ & $48.31(0.05)$ & $49.06(0.17)$ & $48.54(0.44)$ & $49.13(0.04)$ & $49.45(0.15)$ \\
\hline & $\Delta V\left(\mathrm{~km} \mathrm{~s}^{-1}\right)$ & $4.41(0.13)$ & $2.95(0.55)$ & $7.32(1.53)$ & $5.10(0.10)$ & $3.68(0.37)$ \\
\hline & $T_{\mathrm{mb}}(\mathrm{K})$ & 1.19 & 0.26 & 0.14 & 1.16 & 0.29 \\
\hline \multirow{4}{*}{ G014.114-00.574 } & $\mathrm{W}\left(\mathrm{K} \mathrm{km} \mathrm{s}^{-1}\right)$ & $1.50(0.23)^{b}$ & $1.18(0.08)$ & - & $1.71(0.13)^{b}$ & $0.64(0.08)$ \\
\hline & $V_{\mathrm{LSR}}\left(\mathrm{km} \mathrm{s}^{-1}\right)$ & $18.27(0.29)^{b}$ & $19.96(0.12)$ & - & $19.38(0.08)^{b}$ & $20.90(0.21)$ \\
\hline & $\Delta V\left(\mathrm{~km} \mathrm{~s}^{-1}\right)$ & $5.12(0.85)^{b}$ & $3.25(0.24)$ & - & $2.68(0.25)^{b}$ & $3.45(0.51)$ \\
\hline & $T_{\mathrm{mb}}(\mathrm{K})$ & $0.28^{b}$ & 0.34 & - & $0.60^{b}$ & 0.17 \\
\hline \multirow{4}{*}{ G014.194-00.194 } & $\mathrm{W}\left(\mathrm{K} \mathrm{km} \mathrm{s}^{-1}\right)$ & $8.49(0.16)$ & $1.13(0.08)$ & $2.65(0.19)$ & $6.71(0.01)$ & $0.82(0.09)$ \\
\hline & $V_{\mathrm{LSR}}\left(\mathrm{km} \mathrm{s}^{-1}\right)$ & $40.23(0.05)$ & $39.52(0.10)$ & $40.26(0.31)$ & $39.85(0.03)$ & $39.47(0.14)$ \\
\hline & $\Delta V\left(\mathrm{~km} \mathrm{~s}^{-1}\right)$ & $5.77(0.14)$ & $2.86(0.26)$ & $10.24(1.07)$ & $4.35(0.06)$ & $2.84(0.37)$ \\
\hline & $T_{\mathrm{mb}}(\mathrm{K})$ & 1.38 & 0.37 & 0.24 & 1.44 & 0.27 \\
\hline \multirow{4}{*}{ G014.492-00.139 } & $\mathrm{W}\left(\mathrm{K} \mathrm{km} \mathrm{s}^{-1}\right)$ & $2.78(0.12)$ & - & - & $2.30(0.08)$ & $0.29(0.05)$ \\
\hline & $V_{\mathrm{LSR}}\left(\mathrm{km} \mathrm{s}^{-1}\right)$ & $41.96(0.07)$ & - & - & $40.84(0.05)$ & $41.02(0.11)$ \\
\hline & $\Delta V\left(\mathrm{~km} \mathrm{~s}^{-1}\right)$ & $3.64(0.23)$ & - & - & $3.00(0.13)$ & $1.17(0.24)$ \\
\hline & $T_{\mathrm{mb}}(\mathrm{K})$ & 0.72 & - & - & 0.72 & 0.23 \\
\hline \multirow{4}{*}{ G014.632-00.577 } & $\mathrm{W}\left(\mathrm{K} \mathrm{km} \mathrm{s}^{-1}\right)$ & $2.34(0.07)^{b}$ & $1.04(0.07)$ & $1.49(0.15)$ & $2.15(0.08)^{b}$ & $0.91(0.06)$ \\
\hline & $V_{\mathrm{LSR}}\left(\mathrm{km} \mathrm{s}^{-1}\right)$ & $20.32(0.03)^{b}$ & $18.79(0.08)$ & $17.76(0.30)$ & $19.90(0.03)^{b}$ & $18.81(0.07)$ \\
\hline & $\Delta V\left(\mathrm{~km} \mathrm{~s}^{-1}\right)$ & $1.94(0.07)^{b}$ & $2.34(0.21)$ & $6.86(1.03)$ & $1.85(0.08)^{b}$ & $1.94(0.16)$ \\
\hline & $T_{\mathrm{mb}}(\mathrm{K})$ & $1.13^{b}$ & 0.42 & 0.20 & $1.09^{b}$ & 0.44 \\
\hline \multirow{4}{*}{ G018.876-00.489 } & $\mathrm{W}\left(\mathrm{K} \mathrm{km} \mathrm{s}^{-1}\right)$ & $18.63(0.22)$ & $1.60(0.12)$ & - & $12.87(0.14)$ & $1.56(0.12)$ \\
\hline & $V_{\mathrm{LSR}}\left(\mathrm{km} \mathrm{s}^{-1}\right)$ & $66.29(0.04)$ & $66.52(0.14)$ & - & $66.67(0.03)$ & $66.30(0.14)$ \\
\hline & $\Delta V\left(\mathrm{~km} \mathrm{~s}^{-1}\right)$ & $6.22(0.09)$ & $3.84(0.35)$ & - & $5.64(0.08)$ & $3.73(0.37)$ \\
\hline & $T_{\mathrm{mb}}(\mathrm{K})$ & 2.81 & 0.39 & - & 2.14 & 0.39 \\
\hline \multirow{4}{*}{ G309.382-00.134 } & $\mathrm{W}\left(\mathrm{K} \mathrm{km} \mathrm{s}^{-1}\right)$ & $3.36(0.13)^{c}$ & $0.81(0.12)$ & - & $4.30(0.13)$ & - \\
\hline & $V_{\mathrm{LSR}}\left(\mathrm{km} \mathrm{s}^{-1}\right)$ & $-49.06(0.05)^{c}$ & $-50.38(0.22)$ & - & $-50.37(0.07)$ & - \\
\hline & $\Delta V\left(\mathrm{~km} \mathrm{~s}^{-1}\right)$ & $2.82(0.13)^{c}$ & $3.39(0.68)$ & - & $5.34(0.19)$ & - \\
\hline & $T_{\mathrm{mb}}(\mathrm{K})$ & $0.74^{c}$ & 0.23 & - & 0.76 & - \\
\hline \multirow{4}{*}{ G317.867-00.151 } & $\mathrm{W}\left(\mathrm{K} \mathrm{km} \mathrm{s}^{-1}\right)$ & $4.85(0.25)^{c}$ & $1.07(0.12)$ & - & $3.18(0.52)^{c}$ & - \\
\hline & $V_{\mathrm{LSR}}\left(\mathrm{km} \mathrm{s}^{-1}\right)$ & $-38.87(0.09)^{c}$ & $-40.07(0.21)$ & - & $-38.92(0.29)^{c}$ & - \\
\hline & $\Delta V\left(\mathrm{~km} \mathrm{~s}^{-1}\right)$ & $4.18(0.25)^{c}$ & $3.93(0.51)$ & - & $4.30(0.51)^{c}$ & - \\
\hline & $T_{\mathrm{mb}}(\mathrm{K})$ & $1.09^{c}$ & 0.26 & - & $0.70^{c}$ & - \\
\hline \multirow{4}{*}{ G318.779-00.137 } & $\mathrm{W}\left(\mathrm{K} \mathrm{km} \mathrm{s}^{-1}\right)$ & $3.44(0.14)^{c}$ & $0.65(0.10)$ & $0.70(0.13)$ & $4.02(0.12)$ & $0.59(0.10)$ \\
\hline & $V_{\mathrm{LSR}}\left(\mathrm{km} \mathrm{s}^{-1}\right)$ & $-36.45(0.08)^{c}$ & $-38.59(0.25)$ & $-38.06(0.41)$ & $-37.87(0.09)$ & $-37.94(0.33)$ \\
\hline & $\Delta V\left(\mathrm{~km} \mathrm{~s}^{-1}\right)$ & $4.03(0.22)^{c}$ & $3.36(0.62)$ & $4.54(0.99)$ & $5.86(0.21)$ & $3.89(0.74)$ \\
\hline & $T_{\mathrm{mb}}(\mathrm{K})$ & $0.80^{c}$ & 0.18 & 0.14 & 0.65 & 0.14 \\
\hline
\end{tabular}

Notes. ${ }^{(a)}$ Integrated intensity of the line. ${ }^{(b)}$ Spectral lines show two line components. The properties of the first component with the velocity close to the $V_{\mathrm{LSR}}$ are given here. ${ }^{(c)}$ Spectral lines show two line components. The properties of the second component with the velocity close to the $V_{\mathrm{LSR}}$ are given here. ${ }^{(d)}$ Spectral line could not be fitted with single or double Gaussian distribution due to self absorption. The properties of the third component are given here. 
Table B.1. continued.

\begin{tabular}{|c|c|c|c|c|c|c|}
\hline Source & Line properties & \multicolumn{5}{|c|}{ Lines } \\
\hline \multirow{4}{*}{ G320.881-00.397 } & $\mathrm{W}\left(\mathrm{K} \mathrm{km} \mathrm{s}^{-1}\right)$ & $6.27(0.14)$ & $0.93(0.10)$ & - & $3.53(0.12)$ & - \\
\hline & $V_{\mathrm{LSR}}\left(\mathrm{km} \mathrm{s}^{-1}\right)$ & $-44.51(0.06)$ & $-44.48(0.13)$ & - & $-44.83(0.08)$ & - \\
\hline & $\Delta V\left(\mathrm{~km} \mathrm{~s}^{-1}\right)$ & $5.21(0.13)$ & $2.58(0.31)$ & - & $4.45(0.17)$ & - \\
\hline & $T_{\mathrm{mb}}(\mathrm{K})$ & 1.13 & 0.34 & - & 0.75 & - \\
\hline \multirow{4}{*}{ G326.987-00.031 } & $\mathrm{W}\left(\mathrm{K} \mathrm{km} \mathrm{s}^{-1}\right)$ & $4.32(0.13)^{b}$ & $0.56(0.10)$ & - & $3.87(0.12)^{b}$ & $3.07(0.72)$ \\
\hline & $V_{\mathrm{LSR}}\left(\mathrm{km} \mathrm{s}^{-1}\right)$ & $-59.91(0.05)^{b}$ & $-58.39(0.27)$ & - & $-59.39(0.06)^{b}$ & $-59.72(0.19)$ \\
\hline & $\Delta V\left(\mathrm{~km} \mathrm{~s}^{-1}\right)$ & $3.82(0.15)^{b}$ & $2.88(0.70)$ & - & $4.16(0.17)^{b}$ & $3.38(0.28)$ \\
\hline & $T_{\mathrm{mb}}(\mathrm{K})$ & $1.06^{b}$ & 0.18 & - & $0.87^{b}$ & 0.85 \\
\hline \multirow{4}{*}{ G329.029-00.206 } & $\mathrm{W}\left(\mathrm{K} \mathrm{km} \mathrm{s}^{-1}\right)$ & $3.16(0.17)^{d}$ & $1.78(0.11)$ & $4.49(0.18)$ & $0.71(0.08)^{c}$ & $1.36(0.10)$ \\
\hline & $V_{\mathrm{LSR}}\left(\mathrm{km} \mathrm{s}^{-1}\right)$ & $-39.78(0.19)^{d}$ & $-43.30(0.16)$ & $-44.45(0.19)$ & $-46.31(0.05)^{c}$ & $-43.21(0.11)$ \\
\hline & $\Delta V\left(\mathrm{~km} \mathrm{~s}^{-1}\right)$ & $7.49(0.48)^{d}$ & $4.92(0.36)$ & $10.18(0.53)$ & $1.40(0.13)^{c}$ & $3.33(0.29)$ \\
\hline & $T_{\mathrm{mb}}(\mathrm{K})$ & $0.40^{d}$ & 0.34 & 0.41 & $0.45^{c}$ & 0.38 \\
\hline \multirow{4}{*}{ G331.708+00.583-1 } & $\mathrm{W}\left(\mathrm{K} \mathrm{km} \mathrm{s}^{-1}\right)$ & $4.67(0.19)^{b}$ & $1.01(0.10)$ & $2.64(0.18)$ & $3.27(0.26)^{b}$ & $0.73(0.09)$ \\
\hline & $V_{\mathrm{LSR}}\left(\mathrm{km} \mathrm{s}^{-1}\right)$ & $-69.33(0.06)^{b}$ & $-67.20(0.21)$ & $-67.14(0.45)$ & $-68.75(0.10)^{b}$ & $-67.05(0.20)$ \\
\hline & $\Delta V\left(\mathrm{~km} \mathrm{~s}^{-1}\right)$ & $3.96(0.21)^{b}$ & $3.98(0.45)$ & 14.01(1.24) & $3.03(0.22)^{b}$ & $3.44(0.51)$ \\
\hline & $T_{\mathrm{mb}}(\mathrm{K})$ & $1.11^{b}$ & 0.24 & 0.18 & $1.02^{b}$ & 0.20 \\
\hline \multirow{4}{*}{ G331.708+00.583-2 } & $\mathrm{W}\left(\mathrm{K} \mathrm{km} \mathrm{s}^{-1}\right)$ & $3.60(0.10) \mathrm{v}$ & $1.30(0.09)$ & $1.51(0.11)$ & $3.65(0.09)^{b}$ & $0.84(0.08)$ \\
\hline & $V_{\mathrm{LSR}}\left(\mathrm{km} \mathrm{s}^{-1}\right)$ & $-69.57(0.03)^{b}$ & $-67.14(0.15)$ & $-67.11(0.17)$ & $-68.95(0.03)^{b}$ & $-67.17(0.14)$ \\
\hline & $\Delta V\left(\mathrm{~km} \mathrm{~s}^{-1}\right)$ & $2.80(0.10)^{b}$ & $4.08(0.33)$ & $4.99(0.44)$ & $2.72(0.08)^{b}$ & $2.63(0.29)$ \\
\hline & $T_{\mathrm{mb}}(\mathrm{K})$ & $1.21^{b}$ & 0.30 & 0.28 & $1.26^{b}$ & 0.30 \\
\hline \multirow{4}{*}{ G333.656+00.059 } & $\mathrm{W}\left(\mathrm{K} \mathrm{km} \mathrm{s}^{-1}\right)$ & $1.85(0.14)$ & $0.73(0.11)$ & - & $2.26(0.13)$ & - \\
\hline & $V_{\mathrm{LSR}}\left(\mathrm{km} \mathrm{s}^{-1}\right)$ & $-84.20(0.16)$ & $-84.71(0.23)$ & - & $-84.59(0.12)$ & - \\
\hline & $\Delta V\left(\mathrm{~km} \mathrm{~s}^{-1}\right)$ & $4.38(0.39)$ & $2.98(0.57)$ & - & $4.29(0.30)$ & - \\
\hline & $T_{\mathrm{mb}}(\mathrm{K})$ & 0.40 & 0.23 & - & 0.50 & - \\
\hline \multirow{4}{*}{ G335.789+00.174 } & $\mathrm{W}\left(\mathrm{K} \mathrm{km} \mathrm{s}^{-1}\right)$ & $4.82(0.41)^{c}$ & $0.51(0.14)^{c}$ & - & $6.21(0.22)^{c}$ & $1.32(0.10)^{c}$ \\
\hline & $V_{\mathrm{LSR}}\left(\mathrm{km} \mathrm{s}^{-1}\right)$ & $-49.30(0.02)^{c}$ & $-49.45(0.13)^{c}$ & - & $-49.71(0.07)^{c}$ & $-49.73(0.10)^{c}$ \\
\hline & $\Delta V\left(\mathrm{~km} \mathrm{~s}^{-1}\right)$ & $3.95(0.16)^{c}$ & $1.51(0.31)^{c}$ & - & $4.48(0.12)^{c}$ & $2.63(0.20)^{c}$ \\
\hline & $T_{\mathrm{mb}}(\mathrm{K})$ & $1.15^{c}$ & $0.32^{c}$ & - & $1.12^{c}$ & $0.20^{c}$ \\
\hline \multirow{4}{*}{ G336.958-00.224 } & $\mathrm{W}\left(\mathrm{K} \mathrm{km} \mathrm{s}^{-1}\right)$ & $3.75(0.14)$ & $0.28(0.07)$ & - & $2.87(0.10)$ & $0.40(0.07)$ \\
\hline & $V_{\mathrm{LSR}}\left(\mathrm{km} \mathrm{s}^{-1}\right)$ & $-71.54(0.09)$ & $-71.18(0.20)$ & - & $-71.83(0.06)$ & $-71.34(0.20)$ \\
\hline & $\Delta V\left(\mathrm{~km} \mathrm{~s}^{-1}\right)$ & $5.69(0.28)$ & $1.80(0.53)$ & - & $4.01(0.17)$ & $2.18(0.45)$ \\
\hline & $T_{\mathrm{mb}}(\mathrm{K})$ & 0.62 & 0.15 & - & 0.67 & 0.17 \\
\hline \multirow{4}{*}{ G337.176-00.032 } & $\mathrm{W}\left(\mathrm{K} \mathrm{km} \mathrm{s}^{-1}\right)$ & $4.04(0.17)^{d}$ & $0.96(0.10)$ & - & $4.19(0.14)^{c}$ & - \\
\hline & $V_{\mathrm{LSR}}\left(\mathrm{km} \mathrm{s}^{-1}\right)$ & $-69.59(0.09)$ & $-68.15(0.32)$ & - & $-68.77(0.09)^{c}$ & - \\
\hline & $\Delta V\left(\mathrm{~km} \mathrm{~s}^{-1}\right)$ & $5.82(0.34)$ & $6.12(0.79)^{c}$ & - & $6.11(0.30)$ & - \\
\hline & $T_{\mathrm{mb}}(\mathrm{K})$ & 0.65 & 0.15 & - & $0.65^{c}$ & - \\
\hline \multirow{4}{*}{ G337.258-00.101 } & $\mathrm{W}\left(\mathrm{K} \mathrm{km} \mathrm{s}^{-1}\right)$ & $4.43(0.15)$ & $0.43(0.10)$ & - & $4.20(0.14)$ & $0.33(0.09)$ \\
\hline & $V_{\mathrm{LSR}}\left(\mathrm{km} \mathrm{s}^{-1}\right)$ & $-68.47(0.14)$ & $-67.93(0.29)$ & - & $-67.92(0.12)$ & $-68.30(0.29)$ \\
\hline & $\Delta V\left(\mathrm{~km} \mathrm{~s}^{-1}\right)$ & $8.51(0.34)$ & $2.74(0.79)$ & - & $7.25(0.31)$ & $2.30(0.71)$ \\
\hline & $T_{\mathrm{mb}}(\mathrm{K})$ & 0.49 & 0.15 & - & 0.54 & 0.14 \\
\hline \multirow{4}{*}{ G338.786+00.476 } & $\mathrm{W}\left(\mathrm{K} \mathrm{km} \mathrm{s}^{-1}\right)$ & $1.40(0.28)^{c}$ & - & - & $0.59(0.15)^{c}$ & $0.60(0.11)$ \\
\hline & $V_{\mathrm{LSR}}\left(\mathrm{km} \mathrm{s}^{-1}\right)$ & $-66.33(0.06)^{c}$ & - & - & $-66.26(0.11)^{c}$ & $-64.11(0.40)$ \\
\hline & $\Delta V\left(\mathrm{~km} \mathrm{~s}^{-1}\right)$ & $2.21(0.27)^{c}$ & - & - & $1.85(0.39)^{c}$ & $4.44(0.86)$ \\
\hline & $T_{\mathrm{mb}}(\mathrm{K})$ & $0.59^{c}$ & - & - & $0.30^{c}$ & 0.13 \\
\hline \multirow{4}{*}{ G340.784-00.097 } & $\mathrm{W}\left(\mathrm{K} \mathrm{km} \mathrm{s}^{-1}\right)$ & $3.12(0.43)^{b}$ & $0.47(0.08)$ & - & $3.94(0.12)$ & $0.55(0.19)$ \\
\hline & $V_{\mathrm{LSR}}\left(\mathrm{km} \mathrm{s}^{-1}\right)$ & $-102.46(0.15)^{b}$ & $-100.87(0.10)$ & - & $-101.55(0.06)$ & $-101.16(0.25)$ \\
\hline & $\Delta V\left(\mathrm{~km} \mathrm{~s}^{-1}\right)$ & $2.69(0.25)^{b}$ & $1.9(0.32)$ & - & $4.41(0.17)$ & $2.73(1.72)$ \\
\hline & $T_{\mathrm{mb}}(\mathrm{K})$ & $1.09^{b}$ & 0.32 & - & 0.84 & 0.19 \\
\hline \multirow{4}{*}{ G342.484+00.182 } & $\mathrm{W}\left(\mathrm{K} \mathrm{km} \mathrm{s}^{-1}\right)$ & $2.29(0.14)^{c}$ & $0.66(0.07)$ & - & $1.49(0.01)^{c}$ & - \\
\hline & $V_{\mathrm{LSR}}\left(\mathrm{km} \mathrm{s}^{-1}\right)$ & $-40.92(0.07)^{c}$ & $-41.53(0.08)$ & - & $-41.04(0.01)^{c}$ & - \\
\hline & $\Delta V\left(\mathrm{~km} \mathrm{~s}^{-1}\right)$ & $2.53(0.23)^{c}$ & $1.64(0.19)$ & - & $2.09(0.08)^{c}$ & - \\
\hline & $T_{\mathrm{mb}}(\mathrm{K})$ & $0.52^{c}$ & 0.38 & - & $0.67^{c}$ & - \\
\hline
\end{tabular}


Table B.1. continued.

\begin{tabular}{|c|c|c|c|c|c|c|}
\hline Source & Line properties & $\mathrm{HCO}^{+}(1-0)$ & $\mathrm{H}^{13} \mathrm{CO}^{+}(1-0)$ & $\begin{array}{c}\text { Lines } \\
\mathrm{SiO}(2-1)\end{array}$ & $\mathrm{HNC}(1-0)$ & $\mathrm{HN}^{13} \mathrm{C}(1-0)$ \\
\hline \multirow{4}{*}{ G343.756-00.164 } & $\mathrm{W}\left(\mathrm{K} \mathrm{km} \mathrm{s}^{-1}\right)$ & $2.17(0.09)^{b}$ & $1.27(0.08)$ & $1.43(0.10)$ & $2.66(0.08)^{b}$ & $0.98(0.09)$ \\
\hline & $V_{\mathrm{LSR}}\left(\mathrm{km} \mathrm{s}^{-1}\right)$ & $-29.87(0.07)^{b}$ & $-27.69(0.08)$ & $-27.41(0.18)$ & $-29.12(0.03)^{b}$ & $-27.60(0.12)$ \\
\hline & $\Delta V\left(\mathrm{~km} \mathrm{~s}^{-1}\right)$ & $3.32(0.18)^{b}$ & $2.66(0.19)$ & $5.05(0.42)$ & $2.43(0.09)^{b}$ & $2.92(0.33)$ \\
\hline & $T_{\mathrm{mb}}(\mathrm{K})$ & $0.61^{b}$ & 0.45 & 0.27 & $1.03^{b}$ & 0.31 \\
\hline \multirow{4}{*}{ G351.444+00.659 } & $\mathrm{W}\left(\mathrm{K} \mathrm{km} \mathrm{s}^{-1}\right)$ & $28.79(0.20)^{b}$ & $6.06(0.10)$ & $8.62(0.12)$ & $26.10(0.12)$ & $4.73(0.11)$ \\
\hline & $V_{\mathrm{LSR}}\left(\mathrm{km} \mathrm{s}^{-1}\right)$ & $-5.69(0.02)^{b}$ & $-4.35(0.03)$ & $-3.98(0.06)$ & $-4.47(0.01)$ & $-4.31(0.04)$ \\
\hline & $\Delta V\left(\mathrm{~km} \mathrm{~s}^{-1}\right)$ & $5.46(0.05)^{b}$ & $3.89(0.07)$ & $7.42(0.16)$ & $6.03(0.03)$ & $3.89(0.10)$ \\
\hline & $T_{\mathrm{mb}}(\mathrm{K})$ & $4.95^{b}$ & 1.46 & 1.09 & 4.06 & 1.14 \\
\hline \multirow{4}{*}{ G351.571+00.762 } & $\mathrm{W}\left(\mathrm{K} \mathrm{km} \mathrm{s}^{-1}\right)$ & $1.77(0.06)^{c}$ & $0.99(0.10)$ & - & $1.97(0.08)$ & $0.38(0.06)$ \\
\hline & $V_{\mathrm{LSR}}\left(\mathrm{km} \mathrm{s}^{-1}\right)$ & $-2.58(0.06)^{c}$ & $-3.12(0.10)$ & - & $-3.06(0.06)$ & $-2.79(0.10)$ \\
\hline & $\Delta V\left(\mathrm{~km} \mathrm{~s}^{-1}\right)$ & $2.10(<0.01)^{c}$ & $2.25(0.32)$ & - & $2.83(0.13)$ & $1.30(0.23)$ \\
\hline & $T_{\mathrm{mb}}(\mathrm{K})$ & $0.41^{c}$ & 0.41 & - & 0.65 & 0.28 \\
\hline \multirow{4}{*}{ G353.066+00.452-1 } & $\mathrm{W}\left(\mathrm{K} \mathrm{km} \mathrm{s}^{-1}\right)$ & $2.87(0.09)^{c}$ & $0.82(0.10)$ & - & $2.55(0.08)^{c}$ & $0.53(0.11)$ \\
\hline & $V_{\mathrm{LSR}}\left(\mathrm{km} \mathrm{s}^{-1}\right)$ & $2.25(0.04)^{c}$ & $1.62(0.14)$ & - & $1.68(0.05)^{c}$ & $1.56(0.26)$ \\
\hline & $\Delta V\left(\mathrm{~km} \mathrm{~s}^{-1}\right)$ & $2.50(<0.01)^{c}$ & $2.24(0.31)$ & - & $2.70(<0.01)^{c}$ & $2.63(0.63)$ \\
\hline & $T_{\mathrm{mb}}(\mathrm{K})$ & $1.08^{c}$ & 0.34 & - & $0.89^{c}$ & 0.19 \\
\hline \multirow{4}{*}{ G353.066+00.452-2 } & $\mathrm{W}\left(\mathrm{K} \mathrm{km} \mathrm{s}^{-1}\right)$ & $4.73(0.11)^{b}$ & $1.00(0.09)$ & - & $4.41(0.09)^{b}$ & $0.36(0.09)$ \\
\hline & $V_{\mathrm{LSR}}\left(\mathrm{km} \mathrm{s}^{-1}\right)$ & $-2.89(0.03)^{b}$ & $-1.80(0.08)$ & - & $-2.25(0.04)^{b}$ & $-1.68(0.22)$ \\
\hline & $\Delta V\left(\mathrm{~km} \mathrm{~s}^{-1}\right)$ & $2.36(0.06)^{b}$ & $1.74(0.18)$ & - & $3.04(0.02)^{b}$ & $1.58(0.42)$ \\
\hline & $T_{\mathrm{mb}}(\mathrm{K})$ & $1.89^{b}$ & 0.54 & - & $1.36^{b}$ & 0.21 \\
\hline \multirow{4}{*}{ G353.066+00.452-3 } & $\mathrm{W}\left(\mathrm{K} \mathrm{km} \mathrm{s}^{-1}\right)$ & $5.74(0.12)$ & $0.68(0.08)$ & - & $2.88(0.10)$ & - \\
\hline & $V_{\mathrm{LSR}}\left(\mathrm{km} \mathrm{s}^{-1}\right)$ & $-3.11(0.03)$ & $-2.75(0.09)$ & - & $-2.86(0.04)$ & - \\
\hline & $\Delta V\left(\mathrm{~km} \mathrm{~s}^{-1}\right)$ & $2.94(0.08)$ & $1.48(0.23)$ & - & $2.32(0.09)$ & - \\
\hline & $T_{\mathrm{mb}}(\mathrm{K})$ & 1.84 & 0.43 & - & 1.16 & - \\
\hline \multirow{4}{*}{ G353.417-00.079_1 } & $\mathrm{W}\left(\mathrm{K} \mathrm{km} \mathrm{s}^{-1}\right)$ & $11.49(0.32)$ & $1.82(0.18)$ & - & $7.34(0.25)$ & $0.40(0.10)$ \\
\hline & $V_{\mathrm{LSR}}\left(\mathrm{km} \mathrm{s}^{-1}\right)$ & $-50.52(0.13)$ & $-49.76(0.23)$ & - & $-49.84(0.12)$ & $-50.24(0.34)$ \\
\hline & $\Delta V\left(\mathrm{~km} \mathrm{~s}^{-1}\right)$ & $10.17(0.42)$ & $5.29(0.68)$ & - & $7.58(0.35)$ & $2.38(0.55)$ \\
\hline & $T_{\mathrm{mb}}(\mathrm{K})$ & 1.06 & 0.32 & - & 0.91 & 0.16 \\
\hline \multirow{4}{*}{ G353.417-00.079_2 } & $\mathrm{W}\left(\mathrm{K} \mathrm{km} \mathrm{s}^{-1}\right)$ & $0.89(0.17)^{c}$ & $0.46(0.14)$ & - & - & - \\
\hline & $V_{\mathrm{LSR}}\left(\mathrm{km} \mathrm{s}^{-1}\right)$ & $-58.30(0.14)^{c}$ & $-57.23(0.90)$ & - & - & - \\
\hline & $\Delta V\left(\mathrm{~km} \mathrm{~s}^{-1}\right)$ & $2.27(0.39)^{c}$ & $4.76(1.75)$ & - & - & - \\
\hline & $T_{\mathrm{mb}}(\mathrm{K})$ & $0.37^{c}$ & 0.09 & - & - & - \\
\hline \multirow{4}{*}{ G354.944-00.537 } & $\mathrm{W}\left(\mathrm{K} \mathrm{km} \mathrm{s}^{-1}\right)$ & $1.67(0.13)^{b}$ & $0.68(0.07)$ & - & $1.48(0.09)^{b}$ & $0.32(0.07)$ \\
\hline & $V_{\mathrm{LSR}}\left(\mathrm{km} \mathrm{s}^{-1}\right)$ & $-6.85(0.07)^{b}$ & $-5.90(0.11)$ & - & $-6.54(0.05)^{b}$ & $-6.14(0.23)$ \\
\hline & $\Delta V\left(\mathrm{~km} \mathrm{~s}^{-1}\right)$ & $2.23(0.18)^{b}$ & $1.98(0.22)$ & - & $1.74(0.12)^{b}$ & $1.78(0.44)$ \\
\hline & $T_{\mathrm{mb}}(\mathrm{K})$ & $0.70^{b}$ & 0.32 & - & $0.80^{b}$ & 0.17 \\
\hline
\end{tabular}


A\&A 620, A158 (2018)

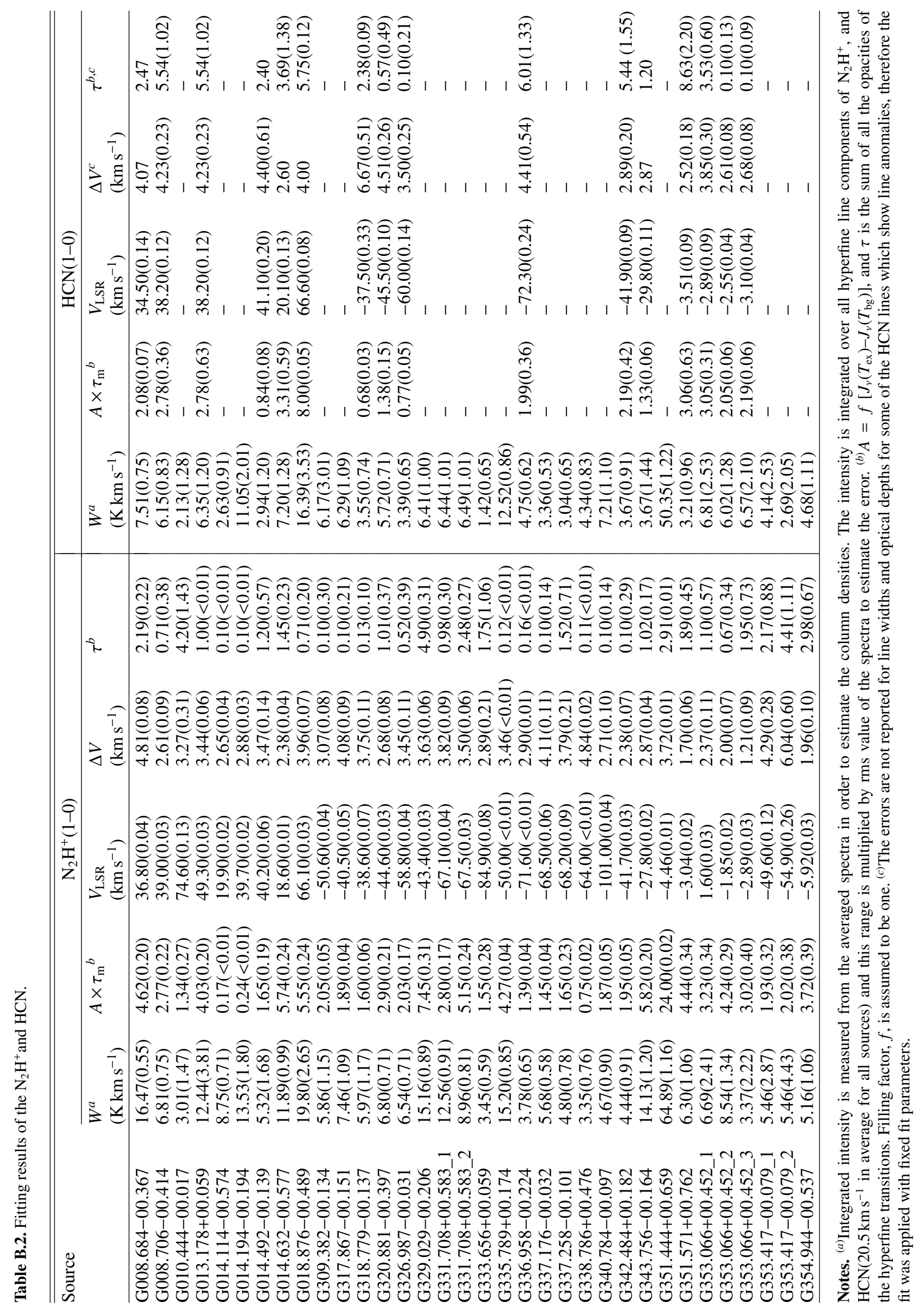


Table B.3. Optical depth measurements for $\mathrm{HCO}^{+}, \mathrm{H}^{13} \mathrm{CO}^{+}, \mathrm{HNC}$, and $\mathrm{HN}^{13} \mathrm{C}$.

\begin{tabular}{lllll}
\hline \hline Source & $\tau_{\mathrm{HCO}^{+}}$ & $\tau_{\mathrm{H}^{13} \mathrm{CO}^{+}}{ }^{*}$ & $\tau_{\mathrm{HNC}}$ & $\tau_{\mathrm{HN}^{13} \mathrm{C}^{*}}$ \\
\hline G008.684-00.367 & $18.64(0.02)$ & 0.36 & $103.15(0.20)$ & 2.00 \\
G008.706-00.414 & $14.55(0.07)$ & 0.28 & $15.81(0.11)$ & 0.30 \\
G010.444-00.017 & - & - & - & - \\
G013.178+00.059 & $12.69(0.05)$ & 0.25 & $15.00(0.08)$ & 0.29 \\
G014.114-00.574 & - & - & $17.04(0.01)$ & 0.33 \\
G014.194-00.194 & $15.94(<0.01)$ & 0.31 & $10.62(<0.01)$ & 0.21 \\
G014.492-00.139 & - & - & $19.46(0.11)$ & 0.38 \\
G014.632-00.577 & $23.91(0.06)$ & 0.46 & $26.94(0.09)$ & 0.52 \\
G018.876-00.489 & $7.758(0.03)$ & 0.15 & $10.63(0.05)$ & 0.20 \\
G309.382-00.134 & $11.36(0.05)$ & 0.22 & - & - \\
G317.867-00.151 & $14.15(0.06)$ & 0.27 & - & - \\
G318.779-00.137 & $13.24(0.06)$ & 0.25 & $12.81(0.09)$ & 0.24 \\
G320.881-00.397 & $18.33(0.10)$ & 0.36 & - & - \\
G326.987-00.031 & $9.67(0.04)$ & 0.19 & $11.79(0.08)$ & 0.22 \\
G329.029-00.206 & $30.09(0.03)$ & 0.59 & $80.05(0.12)$ & 1.57 \\
G331.708+00.583_1 & $12.60(0.06)$ & 0.25 & $11.29(0.08)$ & 0.22 \\
G331.708+00.583_2 & $14.52(0.03)$ & 0.28 & $13.97(0.04)$ & 0.27 \\
G333.656+00.059 & $43.10(0.32)$ & 0.86 & - & - \\
G335.789+00.174 & $17.14(<0.01)$ & 0.33 & $24.13(<0.01)$ & 0.45 \\
G336.958-00.224 & $14.41(0.01)$ & 0.28 & $15.50(0.01)$ & 0.29 \\
G337.176-00.032 & $13.86(0.09)$ & 0.26 & - & - \\
G337.258-00.101 & $18.46(0.12)$ & 0.36 & $15.21(0.14)$ & 0.30 \\
G338.786+00.476 & - & - & $17.97(0.01)$ & 0.34 \\
G340.784-00.097 & $18.05(0.08)$ & 0.35 & $13.55(0.09)$ & 0.26 \\
G342.484+00.182 & $30.78(0.14)$ & 0.59 & - & - \\
G343.756-00.164 & $70.28(0.24)$ & 1.34 & $18.52(0.04)$ & 0.36 \\
G351.444+00.659 & $18.16(<0.01)$ & 0.35 & $17.43(<0.01)$ & 0.33 \\
G351.571+00.762 & $37.36(0.14)$ & 0.73 & $29.03(0.16)$ & 0.56 \\
G353.066+00.452_1 & $19.37(0.16)$ & 0.38 & $12.43(0.15)$ & 0.25 \\
G353.066+00.452_2 & $17.48(0.08)$ & 0.34 & $8.86(0.06)$ & 0.17 \\
G353.066+00.452_3 & $10.64(0.06)$ & 0.21 & - & - \\
G353.417-00.079_1 & $18.08(0.07)$ & 0.36 & $31.22(0.17)$ & 0.62 \\
G353.417-00.079_2 & $13.95(0.04)$ & 0.28 & - & - \\
G354.944-00.537 & $30.91(0.10)$ & 0.61 & $12.14(0.06)$ & 0.24 \\
\hline Mean & $20.32(0.02)$ & $0.39(<0.01)$ & $23.19(0.02)$ & $0.45(<0.01)$ \\
Median & $18.08(<0.01)$ & $0.35(<0.01)$ & $15.50(0.01)$ & $0.30(<0.01)$ \\
Std. & 0.24 & 14.02 & 0.27 \\
\hline & & & &
\end{tabular}

Notes. ${ }^{(*)}$ The errors for optically thin lines are $<0.01$. For the error calculations the Uncertainties: a Python package for calculations with uncertainties by Eric O. Lebigot was used (http://pythonhosted.org/uncertainties/). The errors on optical depths, $T_{\mathrm{ex}}$, and integrated intensities are included in the error calculation of column densities. 
Table B.4. Column density of $\mathrm{HCO}^{+}, \mathrm{H}^{13} \mathrm{CO}^{+}, \mathrm{SiO}, \mathrm{HNC}, \mathrm{HN}^{13} \mathrm{C}, \mathrm{N}_{2} \mathrm{H}^{+}$, and $\mathrm{HCN}$, and $T_{\text {ex }}$ calculated from $\mathrm{N}_{2} \mathrm{H}^{+}$line.

\begin{tabular}{|c|c|c|c|c|c|c|c|c|}
\hline Source & $\begin{array}{l}\mathrm{N}\left(\mathrm{HCO}^{+}\right) \\
\left(\times 10^{14} \mathrm{~cm}^{-2}\right)\end{array}$ & $\begin{array}{l}\mathrm{N}\left(\mathrm{H}^{13} \mathrm{CO}^{+}\right) \\
\left(\times 10^{12} \mathrm{~cm}^{-2}\right)\end{array}$ & $\begin{array}{l}\mathrm{N}(\mathrm{SiO}) \\
\left(\times 10^{12} \mathrm{~cm}^{-2}\right)\end{array}$ & $\begin{array}{l}\mathrm{N}(\mathrm{HNC}) \\
\left(\times 10^{14} \mathrm{~cm}^{-2}\right)\end{array}$ & $\begin{array}{l}\mathrm{N}\left(\mathrm{HN}^{13} \mathrm{C}\right) \\
\left(\times 10^{12} \mathrm{~cm}^{-2}\right)\end{array}$ & $\begin{array}{l}\mathrm{N}\left(\mathrm{N}_{2} \mathrm{H}^{+}\right) \\
\left(\times 10^{12} \mathrm{~cm}^{-2}\right)\end{array}$ & $\begin{array}{l}\mathrm{N}(\mathrm{HCN}) \\
\left(\times 10^{12} \mathrm{~cm}^{-2}\right)\end{array}$ & $\begin{array}{l}T_{\mathrm{ex}}\left(\mathrm{N}_{2} \mathrm{H}^{+}\right)^{a} \\
(\mathrm{~K})\end{array}$ \\
\hline G008.684-00.367 & $1.4(0.1)^{e}$ & $3.6(0.2)$ & $1.4(0.2)$ & $3.2(0.5)$ & $11.7(0.8)$ & $55.8(6.1)$ & $30.0(3.0)$ & $5.1(0.2)$ \\
\hline G008.706-00.414 & $1.2(0.1)$ & $1.3(0.2)$ & $0.6(0.1)$ & $1.3(0.2)$ & $1.4(0.2)$ & $9.3(1.4)$ & $59.7(16.1)$ & $7.0(2.2)^{b}$ \\
\hline G010.444-0<0.117 & $0.1(<0.1)$ & $5.2(-)^{c}$ & $3.0(-)$ & $0.1(<0.1)$ & $10.8(-)$ & $56.3(44.6)$ & $8.0(5.3)$ & $3.1(0.1)$ \\
\hline G013.178+0<0.159 & $0.7(<0.1)$ & $1.0(0.1)$ & $0.5(0.1)$ & $1.3(0.1)$ & $2.6(0.2)$ & $15.4(4.7)$ & $9.3(1.8)$ & 7.1(1.0) \\
\hline G014.114-00.574 & $<0.1(<0.1)$ & $1.6(0.1)$ & $1.0(-)$ & $0.4(<0.1)$ & $1.8(0.2)$ & $13.0(1.1)$ & $4.1(1.4)$ & $4.7(<0.1)$ \\
\hline G014.194-00.194 & $1.5(<0.1)$ & $1.6(0.1)$ & $2.3(0.2)$ & $0.9(<0.1)$ & $2.0(0.2)$ & $18.0(2.4)$ & $16.1(2.9)$ & $5.4(<0.1)$ \\
\hline G014.492-00.139 & $<0.1(<0.1)$ & $1.6(-)$ & $1.0(-)$ & $0.7(0.1)$ & $1.1(0.3)$ & $28.0(14.9)$ & $14.9(6.5)$ & $3.8(0.3)$ \\
\hline G014.632-00.577 & $0.6(<0.1)$ & $1.4(0.1)$ & $1.0(0.1)$ & $0.8(<0.1)$ & $2.1(0.1)$ & $27.9(3.4)$ & $39.9(15.3)$ & $7.0(0.7)$ \\
\hline G018.876-00.489 & $1.6(0.1)$ & $2.1(0.2)$ & $0.3(-)$ & $2.2(0.3)$ & $3.9(0.4)$ & $26.9(4.3)$ & $165.5(40.4)$ & $11.0(2.3)$ \\
\hline G309.382-00.134 & $0.4(<0.1)$ & $1.1(0.2)$ & $0.4(-)$ & $1.8(0.3)$ & $1.6(-)$ & $8.0(1.8)$ & $10.8(5.5)$ & $23.8(62.3)^{b}$ \\
\hline G317.867-00.151 & $0.8(0.1)$ & $1.5(0.2)$ & $0.6(-)$ & $1.3(0.3)$ & $1.4(-)$ & $10.1(1.9)$ & $11.0(2.5)$ & $22.1(40.4)^{b}$ \\
\hline G318.779-00.137 & $0.5(0.1)$ & $0.9(0.2)$ & $0.5(0.1)$ & $0.8(0.1)$ & $1.5(0.3)$ & $8.1(1.8)$ & $16.3(4.2)$ & $15.7(9.6)^{b}$ \\
\hline G320.881-00.397 & $1.2(0.1)$ & $1.3(0.2)$ & $0.7(-)$ & $1.4(<0.1)$ & $1.7(-)$ & $13.8(3.3)$ & $8.3(1.0)$ & $5.9(1.1)$ \\
\hline G326.987-00.031 & $0.5(0.1)$ & $0.7(0.2)$ & $1.1(-)$ & $0.6(0.2)$ & $1.0(0.2)$ & $8.9(1.4)$ & $5.9(1.4)$ & $7.0(3.0)^{b}$ \\
\hline G329.029-00.206 & $0.4(<0.1)$ & $3.4(0.2)$ & $4.9(0.3)$ & $0.8(0.1)$ & $7.9(0.6)$ & $116.2(12.5)$ & $10.2(1.6)$ & $4.5(0.1)$ \\
\hline G331.708+00.583_1 & $0.6(<0.1)$ & $1.3(0.1)$ & $2.1(0.3)$ & $0.5(<0.1)$ & $1.7(0.2)$ & 16.1(1.4) & $9.3(1.5)$ & $5.9(0.9)$ \\
\hline G331.708+00.583_2 & $0.6(<0.1)$ & $1.9(0.1)$ & $1.4(0.1)$ & $0.7(<0.1)$ & $2.2(0.2)$ & $33.5(4.9)$ & $9.6(1.5)$ & $5.1(0.3)$ \\
\hline G333.656+00.059 & $1.4(0.6)$ & $2.1(0.9)$ & $1.2(-)$ & $2.5(0.7)$ & $5.3(-)$ & $15.1(11.8)$ & $19.6(14.6)$ & $3.8(0.6)$ \\
\hline G335.789+00.174 & $2.1(0.2)$ & $1.6(0.5)$ & $1.9(-)$ & $6.3(0.2)$ & $7.4(0.5)$ & $47.7(2.7)$ & $55.8(3.9)$ & $38.0(0.3)$ \\
\hline G336.958-00.224 & $0.6(<0.1)$ & $0.4(0.1)$ & $0.9(-)$ & $0.8(<0.1)$ & $1.0(0.2)$ & $5.3(0.9)$ & $8.8(1.2)$ & $12.1(0.3)$ \\
\hline G337.176-00.132 & $0.6(0.1)$ & $1.3(0.2)$ & $0.3(-)$ & $1.4(0.2)$ & $1.1(-)$ & $7.7(1.2)$ & $5.9(1.3)$ & $17.7(20.8)^{b}$ \\
\hline G337.258-00.101 & $1.3(0.3)$ & $0.9(0.3)$ & $2.2(-)$ & $1.0(0.2)$ & $1.2(0.4)$ & $17.1(9.5)$ & $5.4(1.6)$ & $4.0(0.6)$ \\
\hline G338.786+00.476 & $<0.1(<0.1)$ & $0.2(-)$ & $0.2(-)$ & $0.2(<0.1)$ & $1.4(0.3)$ & $4.4(1.0)$ & 7.3(1.4) & $10.1(0.2)$ \\
\hline G340.784-00.197 & $0.6(0.1)$ & $0.7(0.1)$ & $1.2(-)$ & $0.9(0.1)$ & $1.4(0.5)$ & $6.3(1.4)$ & $12.6(2.7)$ & $21.9(25.9)^{b}$ \\
\hline G342.484+00.182 & $0.8(0.1)$ & $1.1(0.2)$ & $1.0(-)$ & $0.9(0.1)$ & $1.7(-)$ & $6.0(1.4)$ & $35.0(14.1)$ & $22.7(56.1)^{b}$ \\
\hline G343.756-00.164 & $1.6(0.1)$ & $2.6(0.2)$ & $1.0(0.1)$ & $0.7(<0.1)$ & $2.3(0.2)$ & $28.6(2.8)$ & $9.9(3.9)$ & $8.8(1.0)$ \\
\hline G351.444+00.659 & $5.9(<0.1)$ & $8.8(0.1)$ & $6.4(0.1)$ & $7.5(<0.1)$ & $11.9(0.3)$ & $275.7(5.0)$ & $89.8(2.2)$ & $11.4(<0.1)$ \\
\hline G351.571+00.762 & $0.7(0.1)$ & $1.7(0.2)$ & $1.1(-)$ & $0.7(<0.1)$ & $0.9(0.2)$ & $18.7(5.2)$ & $40.5(15.9)$ & $5.4(0.6)$ \\
\hline G353.066+00.452_1 & $0.6(0.1)$ & $1.1(0.2)$ & $0.5(-)$ & $0.4(<0.1)$ & $1.2(0.3)$ & $14.0(6.8)$ & $35.7(14.3)$ & $6.0(1.6)$ \\
\hline G353.066+00.452_2 & $0.9(0.1)$ & $1.4(0.2)$ & $0.5(-)$ & $0.6(0.1)$ & $0.9(0.2)$ & $11.6(2.2)$ & $10.5(2.7)$ & $9.5(3.3)^{b}$ \\
\hline G353.066+00.452_3 & $0.8(0.1)$ & $1.8(0.6)$ & $0.7(-)$ & $0.8(0.1)$ & $2.2(-)$ & $11.8(9.2)$ & $10.4(3.5)$ & $4.5(0.7)$ \\
\hline G353.417-00.179_1 & $3.8(0.8)$ & $4.4(1.0)$ & $1.6(-)$ & $4.1(0.7)$ & $3.7(-)$ & $35.0(24.1)$ & $8.5(5.4)$ & $3.7(0.3)$ \\
\hline G353.417-00.179_2 & $0.3(0.1)$ & $1.6(0.6)$ & $3.8(-)$ & $<0.1(<0.1)$ & $8.0(-)$ & $79.8(74.3)$ & $322.6(297.2)$ & $3.3(0.2)$ \\
\hline G354.944-00.537 & $0.7(0.1)$ & $1.4(0.2)$ & $1.3(-)$ & $0.3(<0.1)$ & $1.0(0.3)$ & $27.6(10.2)$ & $7.9(2.0)$ & $4.2(0.3)$ \\
\hline Mean $^{d}$ & $1.1(<0.1)$ & $1.8(0.1)$ & $2.0(<0.1)$ & $1.4(<0.1)$ & $3.0(0.1)$ & $31.7(2.8)$ & $32.8(8.9)$ & $9.7(2.9)$ \\
\hline Median & $0.7(0.1)$ & $1.4(0.1)$ & $1.4(0.2)$ & $0.8(0.1)$ & $1.6(0.2)$ & $15.7(2.5)$ & $10.6(3.1)$ & $6.5(1.7)$ \\
\hline Std. & 1.1 & 1.5 & 1.8 & 1.6 & 3.2 & 50.2 & 61.5 & 7.8 \\
\hline
\end{tabular}

Notes. ${ }^{(a)} T_{\text {ex }}$ is calculated for $\mathrm{N}_{2} \mathrm{H}^{+}$lines as described in Sect. 3.3. ${ }^{(b)}$ Since the errors on $T_{\text {ex }}$ values calculated for these sources are larger than $30 \%$, in column density calculations $22 \mathrm{~K}$ or $11 \mathrm{~K}$ is used for D8, or D24 clumps, respectively. ${ }^{(c)}$ Column densities without errors represent the upper limits for non-detections. In these case, a $\Delta V=2.0 \mathrm{~km} \mathrm{~s}^{-1}$ was assumed and the brightness temperatures were set to three times the rms of the spectrum $\left(T_{\mathrm{mb}}=3 T_{\mathrm{rms}}\right)$. For these cases, no uncertainties are shown in the table. ${ }^{(d)}$ The upper level column density values are excluded from the mean and median calculations. ${ }^{(e)}$ For the error calculations the Uncertainties: a Python package for calculations with uncertainties by Eric O. Lebigot was used (http://pythonhosted.org/uncertainties/). The errors on optical depths, $T_{\text {ex }}$, and integrated intensities are included in the error calculation of column densities. 
Table B.5. Column density ratios.

\begin{tabular}{|c|c|c|c|c|c|}
\hline Source & $\mathrm{N}(\mathrm{HNC}) / \mathrm{N}\left(\mathrm{HCO}^{+}\right)$ & $\mathrm{N}\left(\mathrm{N}_{2} \mathrm{H}^{+}\right) / \mathrm{N}\left(\mathrm{HCO}^{+}\right)$ & $\mathrm{N}\left(\mathrm{N}_{2} \mathrm{H}^{+}\right) / \mathrm{N}(\mathrm{HNC})$ & $\mathrm{N}(\mathrm{HNC}) / \mathrm{N}(\mathrm{HCN})$ & $\mathrm{N}(\mathrm{HCN}) / \mathrm{N}\left(\mathrm{HCO}^{+}\right.$ \\
\hline G008.684-00.367 & $2.3(0.4)$ & $0.4(0.0)$ & $0.2(0.0)$ & $10.7(2.0)$ & $0.2(0.0)$ \\
\hline G008.706-00.414 & $1.1(0.2)$ & $0.1(0.0)$ & $0.1(0.0)$ & $2.2(0.7)$ & $0.5(0.1)$ \\
\hline G010.444-00.017 & $0.5(0.0)$ & $4.9(2.8)$ & $9.8(5.9)$ & $0.7(0.4)$ & $0.7(0.4)$ \\
\hline G013.178+00.059 & $1.7(0.1)$ & $0.2(0.1)$ & $0.1(0.0)$ & $13.6(2.6)$ & $0.1(0.0)$ \\
\hline G014.114-00.574 & $10.9(1.0)$ & $3.6(0.3)$ & $0.3(0.0)$ & $9.8(3.5)$ & $1.1(0.4)$ \\
\hline G014.194-00.194 & $0.6(0.0)$ & $0.1(0.0)$ & $0.2(0.0)$ & $5.8(1.0)$ & $0.1(0.0)$ \\
\hline G014.492-00.139 & $16.0(1.3)$ & $6.0(2.4)$ & $0.4(0.2)$ & $5.0(2.1)$ & $3.2(1.3)$ \\
\hline G014.632-00.577 & $1.4(0.1)$ & $0.5(0.1)$ & $0.4(0.1)$ & $1.9(0.7)$ & $0.7(0.3)$ \\
\hline G018.876-00.489 & $1.4(0.1)$ & $0.2(0.0)$ & $0.1(0.0)$ & $1.3(0.3)$ & $1.0(0.2)$ \\
\hline G309.382-00.134 & $4.3(0.8)$ & $0.2(0.0)$ & $0.0(0.0)$ & $17.1(9.1)$ & $0.3(0.1)$ \\
\hline G317.867-00.151 & $1.7(0.4)$ & $0.1(0.0)$ & $0.1(0.0)$ & $12.0(3.9)$ & $0.1(0.0)$ \\
\hline G318.779-00.137 & $1.6(0.3)$ & $0.2(0.0)$ & $0.1(0.0)$ & $5.1(1.6)$ & $0.3(0.1)$ \\
\hline G320.881-00.397 & $1.1(0.1)$ & $0.1(0.0)$ & $0.1(0.0)$ & $16.7(2.2)$ & $0.1(0.0)$ \\
\hline G326.987-00.031 & $1.3(0.4)$ & $0.2(0.0)$ & $0.2(0.0)$ & $9.9(3.7)$ & $0.1(0.0)$ \\
\hline G329.029-00.206 & $2.1(0.3)$ & $3.0(0.4)$ & $1.4(0.2)$ & $7.9(1.5)$ & $0.3(0.0)$ \\
\hline G331.708+00.583_1 & $0.8(0.1)$ & $0.3(0.0)$ & $0.3(0.0)$ & $5.2(0.9)$ & $0.1(0.0)$ \\
\hline G331.708+00.583_2 & $1.1(0.0)$ & $0.6(0.1)$ & $0.5(0.1)$ & $7.0(1.1)$ & $0.2(0.0)$ \\
\hline $\mathrm{G} 333.656+00.059$ & $1.8(0.3)$ & $0.1(0.0)$ & $0.1(0.0)$ & $12.7(8.6)$ & $0.1(0.1)$ \\
\hline G335.789+00.174 & $3.0(0.3)$ & $0.2(0.0)$ & $0.1(0.0)$ & $11.2(0.9)$ & $0.3(0.0)$ \\
\hline G336.958-00.224 & $1.2(0.1)$ & $0.1(0.0)$ & $0.1(0.0)$ & $8.7(1.2)$ & $0.1(0.0)$ \\
\hline G337.176-00.032 & $2.3(0.4)$ & $0.1(0.0)$ & $0.1(0.0)$ & $24.2(6.5)$ & $0.1(0.0)$ \\
\hline G337.258-00.101 & $0.8(0.1)$ & $0.1(0.0)$ & $0.2(0.1)$ & $18.4(4.0)$ & $0.0(0.0)$ \\
\hline $\mathrm{G} 338.786+00.476$ & $10.8(3.5)$ & $2.9(0.9)$ & $0.3(0.1)$ & $2.2(0.7)$ & $4.8(1.3)$ \\
\hline G340.784-00.097 & $1.4(0.3)$ & $0.1(0.0)$ & $0.1(0.0)$ & $6.8(1.8)$ & $0.2(0.1)$ \\
\hline G342.484+00.182 & $1.1(0.2)$ & $0.1(0.0)$ & $0.1(0.0)$ & $2.5(1.1)$ & $0.4(0.2)$ \\
\hline G343.756-00.164 & $0.4(0.0)$ & $0.2(0.0)$ & $0.4(0.1)$ & $7.2(2.8)$ & $0.1(0.0)$ \\
\hline $\mathrm{G} 351.444+00.659$ & $1.3(0.0)$ & $0.5(0.0)$ & $0.4(0.0)$ & $8.4(0.2)$ & $0.2(0.0)$ \\
\hline G351.571+00.762 & $1.0(0.1)$ & $0.3(0.1)$ & $0.3(0.1)$ & $1.8(0.7)$ & $0.6(0.2)$ \\
\hline G353.066+00.452_1 & $0.7(0.1)$ & $0.2(0.1)$ & $0.3(0.2)$ & $1.2(0.5)$ & $0.6(0.3)$ \\
\hline G353.066+00.452_2 & $0.7(0.1)$ & $0.1(0.0)$ & $0.2(0.0)$ & $6.0(1.8)$ & $0.1(0.0)$ \\
\hline G353.066+00.452_3 & $1.0(0.1)$ & $0.1(0.1)$ & $0.2(0.1)$ & $7.5(2.4)$ & $0.1(0.0)$ \\
\hline G353.417-00.079_1 & $1.1(0.1)$ & $0.1(0.1)$ & $0.1(0.1)$ & $48.1(29.4)$ & $0.0(0.0)$ \\
\hline G353.417-00.079_2 & $0.1(0.0)$ & $2.3(2.0)$ & $27.6(24.0)$ & $0.0(0.0)$ & $9.4(8.7)$ \\
\hline G354.944-00.537 & $0.4(0.0)$ & $0.4(0.1)$ & $1.0(0.3)$ & $3.4(0.8)$ & $0.1(0.0)$ \\
\hline Mean & $2.3(0.1)$ & $0.8(0.1)$ & $1.3(0.7)$ & $8.9(1.0)$ & $0.8(0.3)$ \\
\hline Median & $1.2(0.2)$ & $0.2(<0.1)$ & $0.2(<0.1)$ & $7.1(1.5)$ & $0.2(<0.1)$ \\
\hline Std. & 3.4 & 1.5 & 4.8 & 8.8 & 1.8 \\
\hline
\end{tabular}

